\title{
A biomechanical switch regulates the transition towards homeostasis in oesophageal epithelium
}

\author{
Jamie McGinn ${ }^{1,2}$, Adrien Hallou ${ }^{1,3,4}$, Seungmin Han ${ }^{1,3}$, Kata Krizic ${ }^{5,6}$, Svetlana Ulyanchenko $\rrbracket^{5,6}$, \\ Ramiro Iglesias-Bartolome ${ }^{10}{ }^{7}$, Frances J. England (1) ', Christophe Verstreken', Kevin J. Chalut', \\ Kim B. Jensen $\mathbb{1}^{5,6}$, Benjamin D. Simons $\mathbb{1}^{1,3,8}$ and Maria P. Alcolea $\mathbb{1}^{1,2} \bowtie$
}

\begin{abstract}
Epithelial cells rapidly adapt their behaviour in response to increasing tissue demands. However, the processes that finely control these cell decisions remain largely unknown. The postnatal period covering the transition between early tissue expansion and the establishment of adult homeostasis provides a convenient model with which to explore this question. Here, we demonstrate that the onset of homeostasis in the epithelium of the mouse oesophagus is guided by the progressive build-up of mechanical strain at the organ level. Single-cell RNA sequencing and whole-organ stretching experiments revealed that the mechanical stress experienced by the growing oesophagus triggers the emergence of a bright Krüppel-like factor 4 (KLF4) committed basal population, which balances cell proliferation and marks the transition towards homeostasis in a yes-associated protein (YAP)-dependent manner. Our results point to a simple mechanism whereby mechanical changes experienced at the whole-tissue level are integrated with those sensed at the cellular level to control epithelial cell fate.
\end{abstract}

pithelial tissues possess the remarkable ability to adapt their program of cell fate in response to increasing tissue demands, such as developmental cues and damage ${ }^{1-7}$. Yet, the mechanisms governing transitions between different modes of cell behaviour remain unclear.

To better understand epithelial cell transitions, we focused on one of the first, and most important, transitions that a specified tissue must face: the establishment of homeostasis. In most tissues, development continues after birth, with a rapid but physiological expansion that encompasses the postnatal growth of the animal and culminates in the establishment of homeostasis ${ }^{8,9}$. At this point, proliferation and differentiation must become balanced to ensure tissue maintenance ${ }^{10}$. However, adult homeostatic cells retain a degree of plasticity, being able to reactivate developmental features if needed ${ }^{11}$. Indeed, differentiating or lineage-committed cells in a range of tissues, including the intestine, lung and skin, have been shown to possess the ability to transition back to a stem cell-like state in response to tissue damage ${ }^{6,12,13}$.

The mouse oesophagus represents an ideal model with which to study changes in epithelial cell dynamics at the whole-tissue level due to its simple architecture ${ }^{10}$. Although the processes governing adult tissue maintenance have been studied widely, here, we aim to shed light on the unknown mechanisms that initially shape homeostasis following postnatal development. During embryonic development, the oesophagus originates as a single layer of cuboidal cells that matures into a squamous, multi-layered tissue $^{14}$. The resulting adult oesophagus is lined by a squamous keratinized epithelium, formed by layers of keratinocytes (Fig. 1a). Proliferation is confined to progenitor cells within the basal layer. Upon commitment to differentiation, basal cells exit the cell cycle and stratify into the suprabasal layers, being ultimately shed at the surface. Quantitative studies have proposed that the homeostatic oesophageal epithelium is maintained by a single, functionally equivalent progenitor population that balances the production of proliferating and differentiating cells through stochastic cell fate decisions ${ }^{15}$.

Growing evidence suggest that cell behaviour is not solely regulated by classically known biochemical cues. Instead, cells are able to alter their behaviour in response to a combination of molecular and mechanical stimuli ${ }^{16-27}$. This is exemplified during postnatal development, when tissues growing at their own unique pace generate significant levels of mechanical stress at the organ level ${ }^{24,28}$. In this context, physical forces influence cell adhesion, shape and niche availability and, in return, feedback on tissue growth, organization and maturation ${ }^{26,29}$.

Here, we show that after birth the developing oesophagus grows at a slower pace than the rest of the body, resulting in the build-up of a physiological tensile strain along its longitudinal axis. Tissue-stretching experiments demonstrate the existence of a defined strain threshold that, once reached, promotes the emergence of a bright Krüppel-like factor 4 (KLF4 ${ }^{\text {Bright }}$ ) committed basal cell population, primed for differentiation, in a yes-associated protein (YAP)-dependent fashion. This mechanical transition signals the tilt of progenitor cell fate from a proliferative mode, during tissue expansion, towards the balanced commitment state characteristic of adult homeostasis ${ }^{15}$. Our results reveal that naturally occurring changes in tissue mechanics orchestrate the establishment of tissue homeostasis.

\footnotetext{
'Wellcome-MRC Cambridge Stem Cell Institute, University of Cambridge, Cambridge, UK. ${ }^{2}$ Department of Oncology, University of Cambridge and Cancer Research UK Cambridge Centre, Cambridge, UK. ${ }^{3}$ Wellcome Trust/Cancer Research UK Gurdon Institute, University of Cambridge, Cambridge, UK. ${ }^{4}$ Cavendish Laboratory, Department of Physics, University of Cambridge, Cambridge, UK. ${ }^{5}$ Biotech Research and Innovation Centre (BRIC), University of Copenhagen, Copenhagen, Denmark. ${ }^{6}$ Novo Nordisk Foundation Center for Stem Cell Biology, Faculty of Health and Medical Sciences, University of Copenhagen, Copenhagen, Denmark. ' Laboratory of Cellular and Molecular Biology, Center for Cancer Research, National Cancer Institute, National Institutes of Health, Bethesda, MD, USA. ${ }^{8}$ Department of Applied Mathematics and Theoretical Physics, Centre for Mathematical Sciences, University of Cambridge, Cambridge, UK. $\varpi_{e}$-mail: mpa28@cam.ac.uk
} 


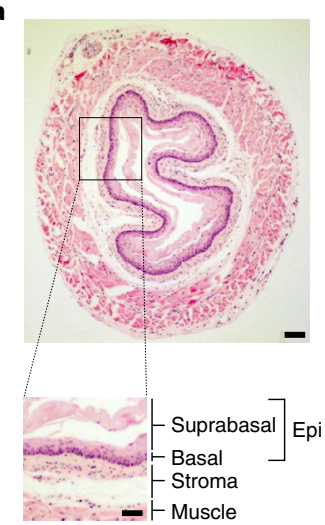

b

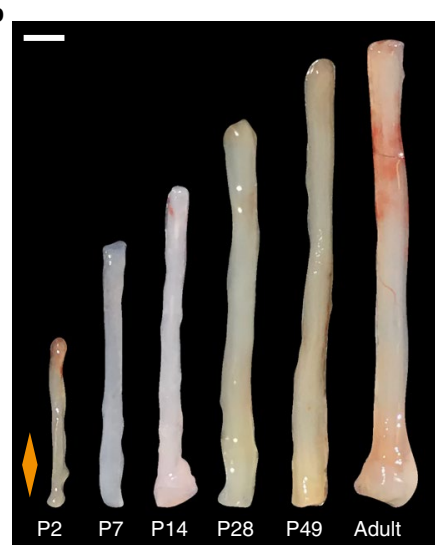

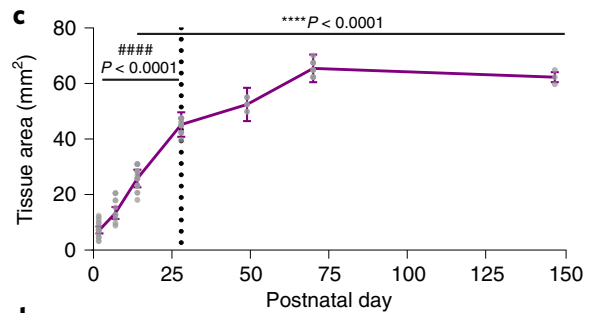

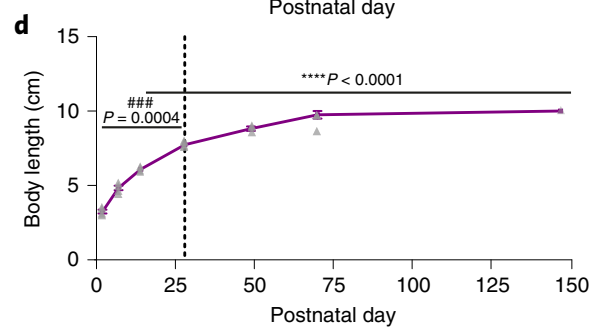

e
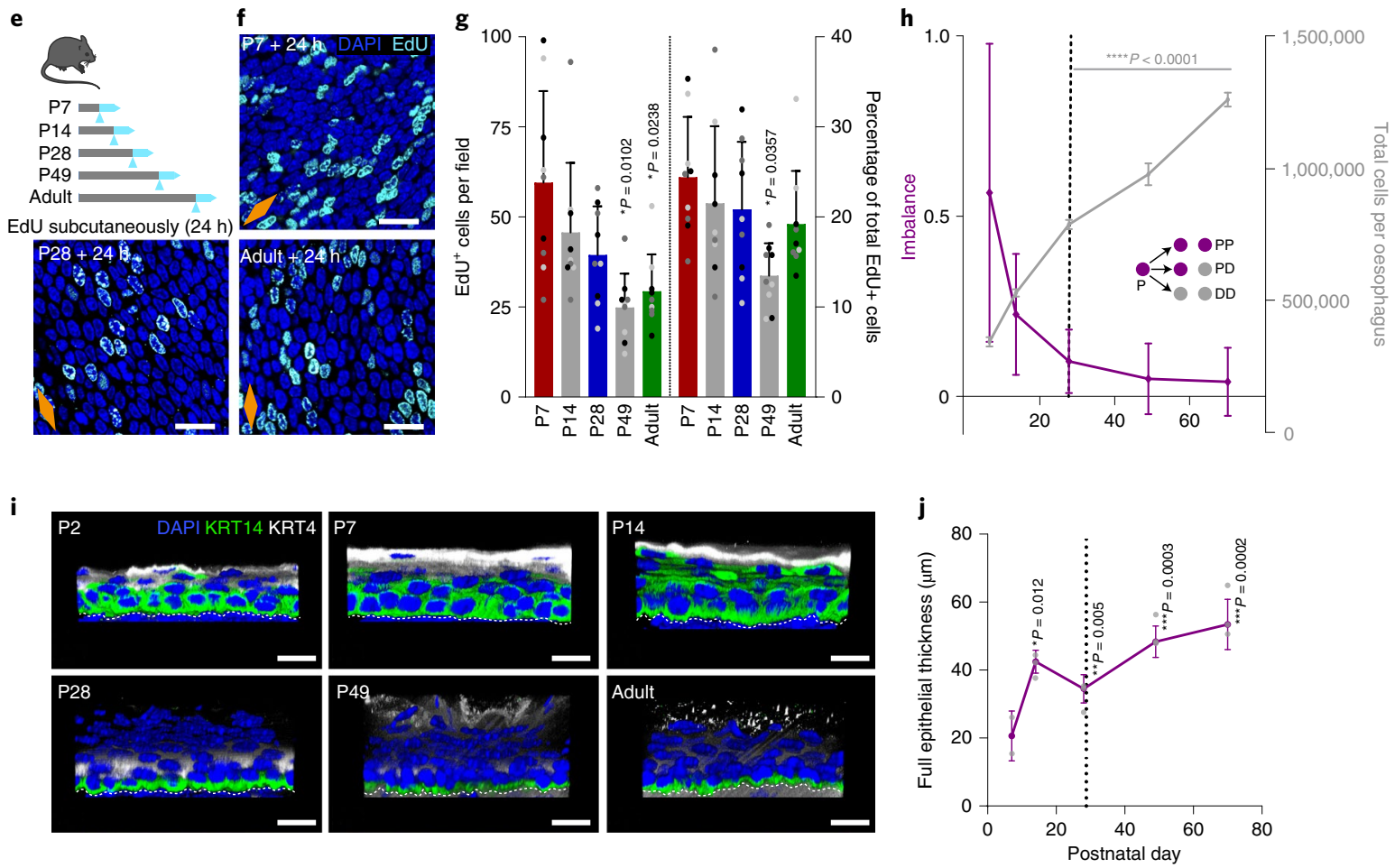

Fig. 1 | The postnatal mouse oesophagus as a model of rapid tissue expansion transitioning towards homeostasis. a, Haematoxylin and eosin-stained section of adult oesophagus. The inset is a magnified image of the main image, showing tissue layers. Epi, epithelium. $\mathbf{b}$, Images of whole oesophagus at different stages of postnatal development. c, Tissue area after longitudinally opening the oesophageal tube ( $n=103$ mice). d, Whole-body growth in length, excluding the tail ( $n=26$ mice). e, In vivo protocol. Mice were treated with a single EdU injection $24 \mathrm{~h}$ before tissue collection at the time points indicated. f, Basal confocal views of EdU+ cells in typical oesophageal epithelial wholemounts from e. $\mathbf{g}$, Quantification of total EdU+ cells per field (left) and percentage of total $\mathrm{EdU}^{+}$cells relative to the number of $\mathrm{DAPI}^{+}$basal cells (right) from e. The data are presented as means \pm s.d. The points show individual measurements (greyscale indicates values from each of $n=3$ mice). $\mathbf{h}$, Fraction of cell fate imbalance, showing the degree to which basal cell fate is biased towards duplication over differentiation and loss (left axis; see Methods and model in Fig. $2 n$ ), and total cell production throughout postnatal development (right axis; see Methods). Over time, oesophageal epithelial cells tilt towards balanced cell fate in line with the model of oesophageal homeostasis by Doupe et al. ${ }^{15}$ (inset; P, progenitor cell; D, differentiating cell). $n=3$ mice. See Supplementary Note 1. i, Representative 3D-rendered confocal z-stacks showing side views of oesophageal epithelial wholemounts throughout postnatal development. The dashed lines indicate the basement membrane. j, Epithelial thickness, including enucleated terminally differentiated layers ( $n=3$ mice). All data were derived from wild-type C57BL/6J mice and are expressed as means \pm s.e.m. unless otherwise stated. Data analysis was performed using one-way ANOVA with Tukey's multiple comparisons test (\#\#\#P<0.001, \#\#\#\#P $<0.0001$ relative to $\mathrm{P} 70 ;{ }^{\star} P<0.05,{ }^{\star \star} P<0.01,{ }^{\star \star \star} P<0.001,{ }^{\star \star \star \star} P<0.0001$ relative to $\mathrm{P} 7$ ). Scale bars, $200 \mu \mathrm{m}$ (main image in a), $100 \mu \mathrm{m}$ (inset in a), $2 \mathrm{~mm}(\mathbf{b})$ and $20 \mu \mathrm{m}$ (f and i). Blue, cyan, green and greyscale stainings represent DAPI, EdU, basal marker KRT14 and the differentiation marker KRT4, respectively. The dotted vertical lines in $\mathbf{c}, \mathbf{d}, \mathbf{h}$ and $\mathbf{j}$ indicate P28. The orange diamonds in $\mathbf{b}$ and $\mathbf{f}$ depict the longitudinal orientation of the oesophagus (as outlined in Extended Data Fig. 1a). Parts of e were drawn using and/or adapting diagrams from Servier Medical Art. See also Extended Data Fig. 1.

\section{Results}

Oesophageal transition towards homeostasis. To study changes associated with the establishment of adult homeostasis, we started by measuring tissue dimensions from postnatal day (P) 2 to P147 (Extended Data Fig. 1a-c). The oesophagus showed a biphasic mode of growth, encompassing the growth of the animal (Fig. 1b-d 
and Extended Data Fig. 1b-d). The initial phase of rapid oesophageal expansion slowed down by $\sim$ P28 and stabilized at P70, coinciding with the establishment of adult homeostasis ${ }^{15}$.

Next, we looked at cell proliferation using short-term in vivo 5-ethynyl-2'-deoxyuridine (EdU) incorporation assays. Quantification of EdU ${ }^{+}$cells after a 24 -h chase revealed a reduction in the proliferative activity of progenitors over time (Fig. 1e-g and Extended Data Fig. 1e). The tilt away from progenitor cell duplication was further revealed through an estimate of fate imbalance (Fig. 1h, Extended Data Fig. 1f, Methods and Supplementary Note 1). The degree of fate imbalance was determined as the ratio of the differential change in total basal cell number to the cell division rate. When the fate imbalance is large, progenitor cell divisions are biased towards a symmetrical duplicative fate outcome (model in Fig. 1h, inset), whereas when the fate imbalance is small, cell duplication is approximately balanced by cell differentiation. During the early postnatal period, we observed a $60 \%$ imbalance towards cell duplication, which drastically fell, reaching a near-balance homeostatic-like state between P28 and adult.

Next, we performed immunostaining for keratin 14 (KRT14) and Keratin 4 (KRT4), which mark the basal and suprabasal layers of the adult oesophageal epithelium, respectively. At early time points, both keratins showed an atypical expression pattern, acquiring their characteristic adult profile by P28 (Fig. 1i and Extended Data Fig. 1g,h). The ongoing maturation of the tissue was further illustrated by the progressive keratinization and cornification of the uppermost luminal layers, as well as increasing tissue thickness (Fig. 1i,j and Extended Data Fig. 1h-k).

These results suggest that, unlike the skin, the squamous epithelium of the oesophagus has not reached full maturation and keratinization by birth ${ }^{30}$. Instead, our data point towards P28 as the approximate age at which the features of the homeostatic oesophageal epithelium become established.

KLF4 marks the emergence of an early committed population. To further understand the transition towards homeostasis, we analysed the expression pattern of key transcription factors associated with cell fate in oesophageal wholemounts by three-dimensional (3D) confocal imaging. These included SRY-box 2 (SOX2; which marks basal progenitor cells in the oesophageal epithelium ${ }^{31,32}$ ) and KLF4 (a well-characterised transcription factor marking suprabasal differentiated cells $^{33-37}$ ). Similar to KRT14 and KRT4 (Fig. 1i), SOX2 and KLF4 expression at early time points was not restricted to their typical basal and suprabasal location in adults, showing an immature pattern of expression. By P28, SOX2 and KLF4 acquired their characteristic adult distribution, reflecting the maturation of the tissue (Fig. 2a).

Interestingly, we noticed a progressive increase in the percentage of basal cells expressing high levels of KLF4 (hereafter, KLF4 bright and dim cells will be referred to as $\mathrm{KLF}^{+}$and $\mathrm{KLF}^{-}$, respectively; Methods). KLF $4{ }^{+}$cells were initially rare, representing $\sim 2-3 \%$ of the basal population. This increased to $\sim 9 \%$ by P28, reaching $\sim 19 \%$ in the adult oesophageal epithelium (Fig. 2a-g and Extended Data Fig. 2a). This resulted in a salt and pepper pattern in the basal progenitor compartment, with $\mathrm{SOX}_{2}{ }^{+}$cells interspersed with $\mathrm{SOX} 2^{+} / \mathrm{KLF}^{+}$ cells (Fig. 2a,b). The temporal changes in basal KLF4 expression, coinciding with the transition window at P28, identified KLF4 as a signature marking the transition towards adult homeostasis.

Since KLF4 is a transcription factor characteristic of differentiated cells, we reasoned that the previously unreported basal $\mathrm{KLF}^{+}$ population identified here could represent a pool of early committed cells transitioning towards differentiation in the oesophageal epithelium (Fig. 2c,d). Consistent with this notion, previous work has shown how targeted deletion of Klf4 in the mouse oesophagus results in increased proliferation in the basal layer ${ }^{33}$. To explore this hypothesis, we initially investigated the proliferative activity of the $\mathrm{KLF}^{+}$basal subpopulation. Short-term EdU incorporation assays (Fig. 2e-h), as well as quantitative imaging analysis of wholemounts obtained from the cell cycle reporter mouse line Fucci2a (R26Fucci2aR; Extended Data Fig. 2b-e), revealed that the KLF4 ${ }^{+}$ population identified in the basal layer of the oesophageal epithelium was in a nonproliferative resting state. Next, we evaluated the expression profile of KRT4 and KRT14 in the $\mathrm{KLF}_{4}^{+-}$basal populations. We noted that, although $\mathrm{KLF}^{+}$basal cells could be found expressing any potential combination of these keratins (Fig. 2i,j), the adult basal KLF4 ${ }^{+}$population was enriched in KRT4, revealing a bias towards differentiation (Fig. 2k). Taken together, the data support the committed state of the KLF4 ${ }^{+}$basal population. The emergence of this committed population by P28 inevitably slows down the rapid tissue expansion that takes place immediately after birth, favouring the balanced proliferation/differentiation typical of adult homeostasis.

Next, we explored a debated question in the field: whether basal progenitors initiate commitment towards differentiation from the

Fig. 2 | KLF4 marks the emergence of an early committed population in the oesophageal epithelium. a, Confocal z-stack side views of typical oesophageal epithelial wholemounts. White arrowheads point to basal KLF4+ cells. The dashed lines represent the basement membrane. The dotted lines represent the upper limit of the oesophageal epithelium. b, Representative basal views of oesophageal epithelial wholemounts from a. c, 3D confocal side views of basal insets from adult oesophageal epithelium, showing basal KLF4+ cells (arrowhead) in top panel detaching from the basement membrane (dotted line) in middle panel and transitioning from basal to suprabasal layers in bottom panel. E-cad, E-cadherin. d, Illustration of transitioning $\mathrm{KLF} 4^{+}$cells (red) as shown in c. e, In vivo protocol. Mice were treated with EdU for $2 \mathrm{~h}$ at the time points indicated (blue arrowheads). $\mathbf{f}$, Basal EdU and KLF4 intensity quantification (a.u.) from e, dotted lines define positive and negative quadrants. Double positive quadrant (shaded blue) highlights the exclusivity of EdU and KLF4 labelling (see Methods). $n=3$ mice. $\mathbf{g}$, Percentage of KLF4+ basal cells, expressed as mean values \pm s.d. Statistical significance was determined by one-way ANOVA with Tukey's multiple comparisons test ( ${ }^{\star \star} P<0.01$ relative to P7). The points show individual measurements (greyscale indicates values from each of $n=3$ mice). $\mathbf{h}$, Basal views of adult oesophageal epithelium from $\mathbf{e}$. The dotted lines depict EdU+ basal cells. i, Basal views of adult oesophageal epithelial wholemounts, showing co-localization of KLF4 (red) with basal (KRT14) and suprabasal markers (KRT4). The white arrowheads point to cells of interest. j, Side-view illustrations depicting KLF4+ (red), KRT14 and KRT4 basal co-localization combinations, as shown in i. k, Percentage of KRT4+ cells within the basal KLF4+ and KLF4- populations. The data represent means \pm s.e.m. ( $n=3$ mice). Statistical significance was determined by two-way ANOVA with Tukey's multiple comparisons test ( ${ }^{\star \star \star \star} P<0.0001$; NS, not significant). I, In vivo protocol. Adult mice were administered a single dose of EdU at 18:00 and sampled after $12 \mathrm{~h}$ to identify post-mitotic EdU ${ }^{+}$basal pairs. $\mathbf{m}$, Representative basal views of adult oesophageal epithelium from $\mathbf{I}$. The dashed lines depict EdU ${ }^{+}$pairs. $\mathbf{n}$, Schematic showing quantitative data on KLF4 expression in EdU ${ }^{+}$pairs from I. Statistical significance was determined by one-way ANOVA with Tukey's multiple comparisons test $\left(n=3\right.$ mice. $\left.{ }^{\star} P<0.05\right)$. Estimates from the oesophageal homeostasis model by Doupe et al. ${ }^{15}$ are shown in parentheses. See Supplementary Note 1. o, Basal confocal views of typical adult oesophageal epithelial wholemounts from $\mathbf{m}$. The dotted lines depict representative pairs. Scale bars, $20 \mu \mathrm{m}(\mathbf{a}, \mathbf{b}, \mathbf{h}$ and $\mathbf{m})$ and $5 \mu \mathrm{m}(\mathbf{c}, \mathbf{i}$ and $\mathbf{o})$. Blue represents DAPI, cyan is EdU, green is SOX2 (a,b) or KRT14 (i), red marks KLF4, and greyscale refers to E-cadh (c) or KRT4 (i). The orange diamonds in $\mathbf{b}, \mathbf{h}, \mathbf{i}, \mathbf{m}$ and $\mathbf{o}$ depict the longitudinal orientation of the oesophagus (Extended Data Fig. 1a). Parts of $\mathbf{e}$ and I were drawn using and/or adapting diagrams from Servier Medical Art. See also Extended Data Fig. 2. 
moment of division, or whether the cell fate outcome is assigned later, following a refractory period in the basal compartment ${ }^{15,38-42}$. For this, we quantified the expression of KLF4 in post-mitotic EdU basal pairs, as marked by a 12 -h EdU chase ${ }^{43}$ (Fig. 21,m). Our results revealed KLF4 levels being reliably detected in EdU ${ }^{+}$sister cells just hours after their physical post-mitotic separation, supporting the notion that fate can be assigned from the time of division (Fig. 2n,o and Supplementary Note 1).

Collectively, we conclude that the emergence of KLF4 in the basal cell compartment defines an early committed population, marking the transition towards homeostasis. These data provide further insight into the temporal resolution of cell fate commitment, a

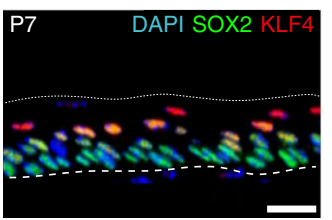

b

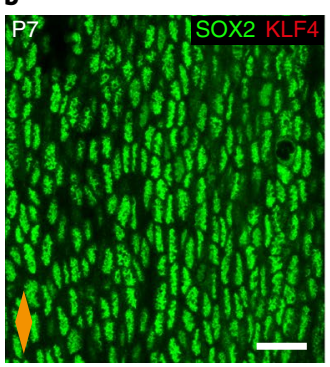

c

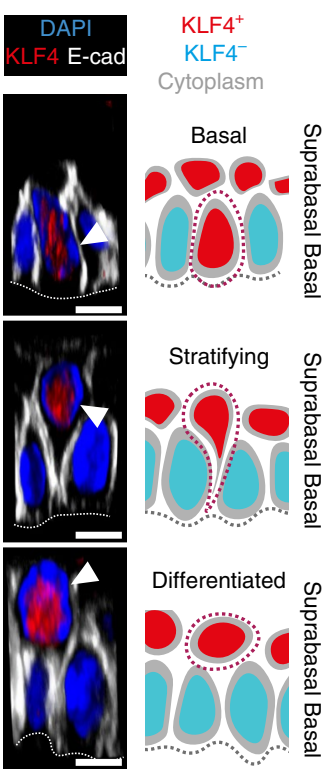

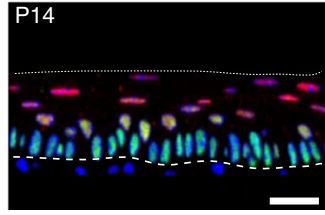
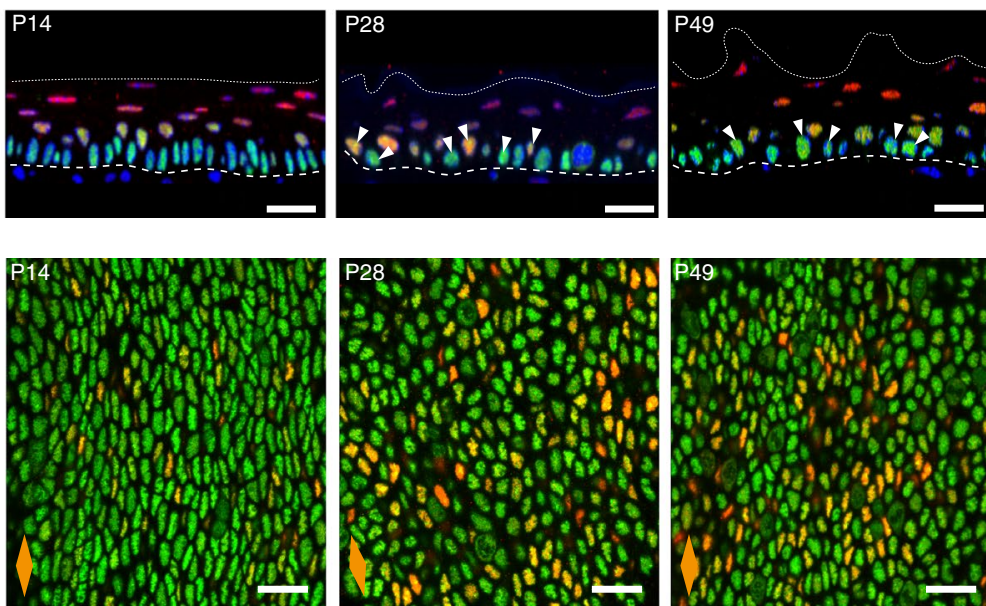

g

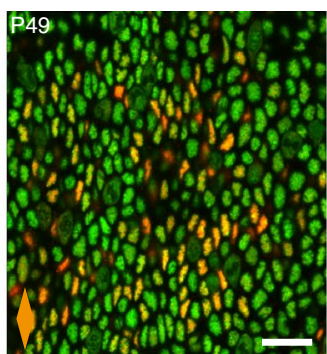

h
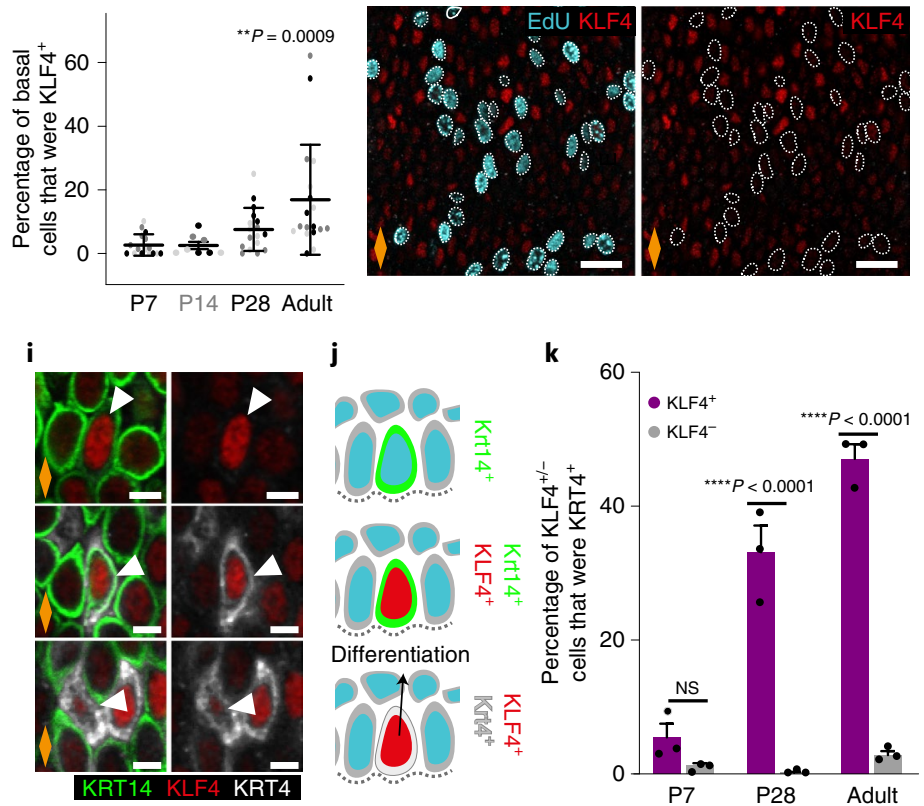

I

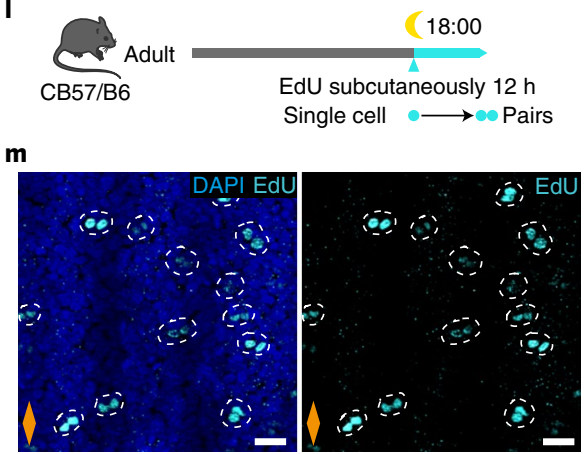

$\mathbf{n}$

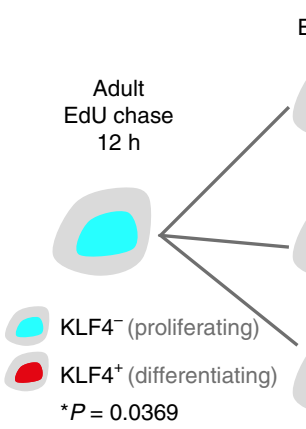

o

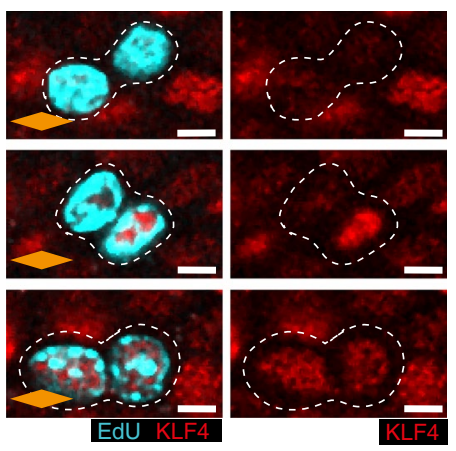


revealing that commitment towards differentiation can be made from the division stage.

Single-cell RNA sequencing (scRNA-seq) captures the onset of homeostasis. To explore the mechanisms regulating the emergence of the $\mathrm{KLF}^{+}{ }^{+}$basal population and the transition to homeostasis, we made use of large-scale transcriptomics at single-cell resolution, focusing on three critical time points spanning the postnatal transition: P7, P28 and adult. Single-cell RNA-seq was performed on the viable epithelial cell fraction $\left(\mathrm{EpCam}^{+} / \mathrm{Cd}_{4} 5^{-}\right)$isolated from the oesophagus of C57BL/6 wild-type mice (Fig. 3a, Extended Data Fig. 3a and Supplementary Table 1). The uniform manifold approximation and projection (UMAP) distribution of the single-cell data revealed a noticeable separation of P7 cells, while P28 and adult cells clustered closely together (Fig. 3b). This confirmed that by P28, although not fully mature, the oesophageal epithelium resembles the adult homeostatic tissue at the transcriptional level. Hence, P28 and adult samples were grouped when exploring gene expression patterns, unless otherwise stated.

The combined dataset underwent unsupervised clustering and annotation using signatures of known lineage and cell cycle markers (Fig. 3c and Extended Data Fig. 3b-i). We identified three different populations for all three time points: (1) actively cycling basal progenitors, defined by increased expression of both basal and cell cycle markers; (2) resting/committed basal cells, with enriched basal signature expression and reduced levels of cycling markers; and (3) differentiated cells, marked by increased expression of genes associated with differentiation, as well as reduced levels of both basal and cell cycle marker genes (Fig. 3c, Extended Data Fig. 3j,k and Methods). Integration analysis of the unsupervised clusters confirmed the equivalence of the assigned populations between time points (Extended Data Fig. 3c and Methods). We concluded that, consistent with the current model ${ }^{15}$, the basal layer of the oesophageal epithelium is formed by a mixture of actively cycling and resting cells.

To shed light on the regulatory processes governing the epithelial transition towards homeostasis, we focused our analysis on the basal cell compartment, where progenitor cells reside. We identified four major patterns defining changes in gene expression over time (Extended Data Fig. 4a,b, Supplementary Table 2 and Methods). Gene Ontology enrichment analysis of the pattern genes revealed a particularly strong WNT signature at P7 (Wnt5b, Fzd2, Sox9, Sfrp2, Lgr5, Dkk3 and Wnt11) (Extended Data Fig. 4b,c). Other signalling genes, with notable differences at P7, included increased expression of insulin-like growth factor-related genes (Igfbp3, Igfbp4, Igf2 and Igf1r), as well as Pdgfa, Fgfr2 and Fgfr3 (Extended Data Fig. 4b,c and Supplementary Table 3), hinting at an active crosstalk between epithelial and mesenchymal cells at early postnatal stages ${ }^{44,45}$. In contrast, later time points were indicative of a maturation process, drifting towards bone morphogenetic protein (BMP)-driven regulation (Bmp3, Bmp4, Btg2, Hoxc8 and Hoxc4) (Extended Data Fig. $4 \mathrm{~b}, \mathrm{~d})^{46-48}$ and showing changes in genes related to protein biogenesis and metabolism (Supplementary Table 3) ${ }^{49,50}$. Consistent with a transition away from the developmental state of the early postnatal oesophagus ${ }^{51-53}$, P28 and adult cells showed an enrichment in genes involved in tissue maintenance and homeostasis, including Cd44, Ly6a and Mt2 (ref. ${ }^{54}$ ). This differed from the P7 epithelium, which presented a marked expression of genes directly associated with epithelial development, wound healing and cancer

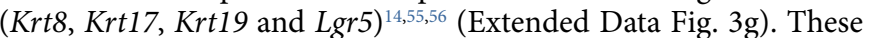
transcriptional profiles reflect the fact that, from P28 onwards, basal cells favour maturation, leaving behind the proliferative state that governs tissue expansion shortly after birth ${ }^{51-53}$.

A subset of related Gene Ontology terms were found to change at both early and later time points. We interpreted these shared changes as an indication of an active switch, suggesting their relevance during this developmental transition. These included Gene Ontology terms associated with extracellular matrix and cytoskeleton organization, cell adhesion and response to mechanical stimulus (Extended Data Fig. 4b-d), all of which are critical for the mechanical stability and behaviour of epithelial cells ${ }^{57,58}$. Indeed, the transcriptional signature of basal cells showed a marked transition from active cytoskeletal remodelling at P7 (Tnc, $P d p n$, Rhoc, Evl, Vcan and Mmp14) to an increased cell adhesion at later time points (Dcn, Lad1, Cfl1, Dstn, Dsc3 and Rhob). To explore whether these processes were related to the emergence of the $\mathrm{KLF}^{+}{ }^{+}$committed population in the basal layer (Fig. 2), we started by assessing Klf4 expression across all clusters and time points. As predicted from tissue observations, Klf4 expression was higher in differentiated clusters, as well as in a subset of the resting basal population that became progressively established from P28 onward, defined by cluster 12 (C12) (Extended Data Fig. 4e). To investigate what makes $\mathrm{C} 12$ distinctive, we further compared the transcriptional profile of this $K l f 4^{\text {high }}$ cluster with the remaining resting basal clusters (C2 and $\mathrm{C} 6$ for $\mathrm{P} 7$ and $\mathrm{C} 1$ and $\mathrm{C} 7$ for P28 and adult; Fig. 3d-g and Supplementary Table 4). We identified three patterns of differential gene expression in the resting basal compartment: (1) pattern 1 (genes elevated at P7); (2) pattern 2 (genes highly expressed in Klf $4^{\text {high }} \mathrm{C} 12$ ); and (3) pattern 3 (genes progressively increasing over time) (Fig. 3f). This analysis showed that Klf4 and Klf4-associated genes, including the downstream targets Krt4, Krt13, Cdkn1a and Cebpb $b^{59-61}$, presented a higher expression level at later time points (P28 and adult; Fig. 3f,g). Additionally, we were able to confirm that the Klf4 signature seen

Fig. 3 | Single-cell transcriptional profiling defines transition towards homeostasis. a, Schematic of scRNA-seq data generation from sorted epithelial cells $\left(\mathrm{EpCam}^{+} / \mathrm{Cd}_{4} 5^{-}\right)$using the $10 \times$ Genomics platform. Cells were isolated from the oesophagus of fifteen P7 mice, twelve P28 mice and nine adult mice, producing three libraries (x10; Chromium) for P7 and P28 mice and six libraries for adult mice. $\mathbf{b}$, Cell distribution in the dimension reduction space UMAP at different postnatal time points. c, UMAPs representing annotated cell types. CB, cycling basal; D, differentiated; RB, resting basal. d, UMAPs representing resting basal cell clusters from Extended Data Fig. 3b. e, Fraction of resting basal cell clusters per time point. The data are expressed as means \pm s.e.m. ( $n=3$ for P7 and P28; $n=6$ for adults). f, Left: heatmap representing the expression of individual genes belonging to distinctive patterns of gene expression in resting basal cells, as defined in $\mathbf{c}$ and $\mathbf{d}$. Numbers of genes are provided in parentheses. For expression values, log ${ }_{2}$-transformed normalized UMIs were scaled and averaged across all cells belonging to each group of resting basal cell clusters. The scale bar denotes the expression range. Right: expression of selected genes related to KLF4, YAP and the response to mechanical stimuli. The colours of gene names denote the relevancy to KLF4, YAP and the response to mechanical stimuli. $\mathbf{g}$, Violin plots showing the expression distribution from resting basal through to differentiated (diff) clusters of selected genes from $\mathbf{f}$ that are related to KLF4 and tissue mechanics. Grey shade highlights resting basal cluster 12, enriched for cells expressing high KIf4. Expression is presented as the $\log _{2}$-transformed normalized UMI. Dashed lines represent mean values. $\mathbf{h}$, Expression of relevant genes along the pseudotime trajectory from resting basal to differentiated cells for P28 and adult. Left: YAP target genes (Cyr61, Ctgf and Thbs7) and genes associated with a response to mechanical stimuli (Cav1, KIf2 and Dcn). Right: KLF4 target genes (KIf4, Krt4, Krt13, Cdnk1a and Cebpb). Gene expression is represented as the auto-scaled, $\log _{2}$-transformed normalized UMI, smoothed using a rolling mean along its trajectory, with a window size of $5 \%$ of cells. The three bars on the top denote the arrangement of cells according to real time points, pseudotime and clusters in Extended Data Fig. 3b, respectively. Parts of a were drawn using and/or adapting diagrams from Servier Medical Art. See also Extended Data Figs. 3 and 4. 
in $\mathrm{C} 12$ is a feature of an emerging committed basal population, which becomes more marked as cells fulfil their differentiation program (C14, C8 and C9; Fig. 3g). Therefore, the Klf4 expression

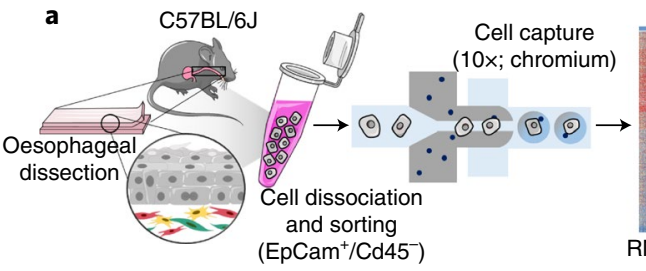

b
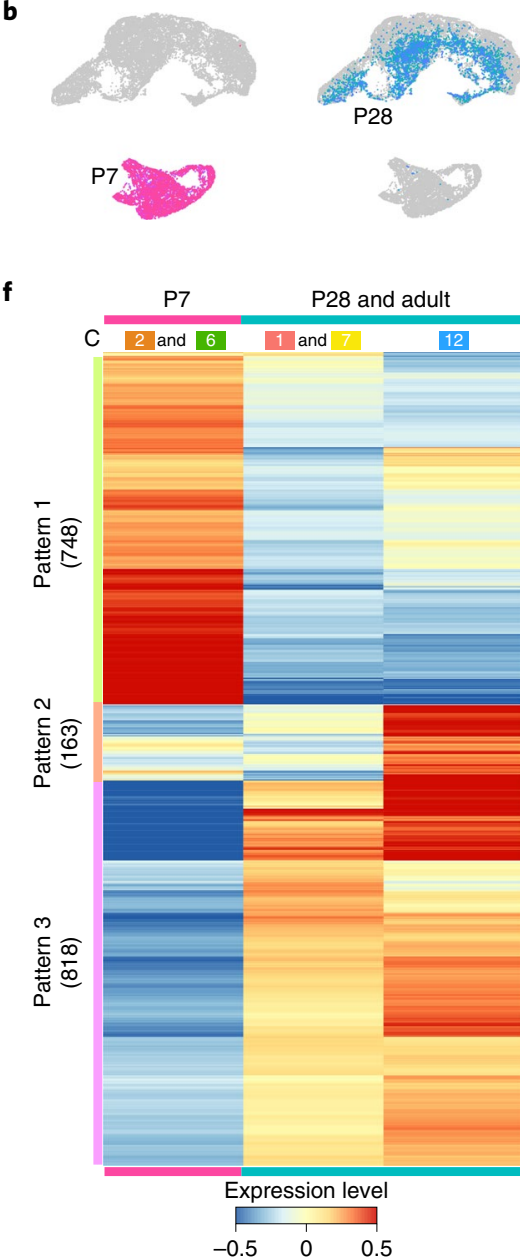

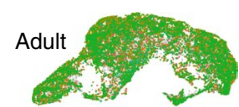

28
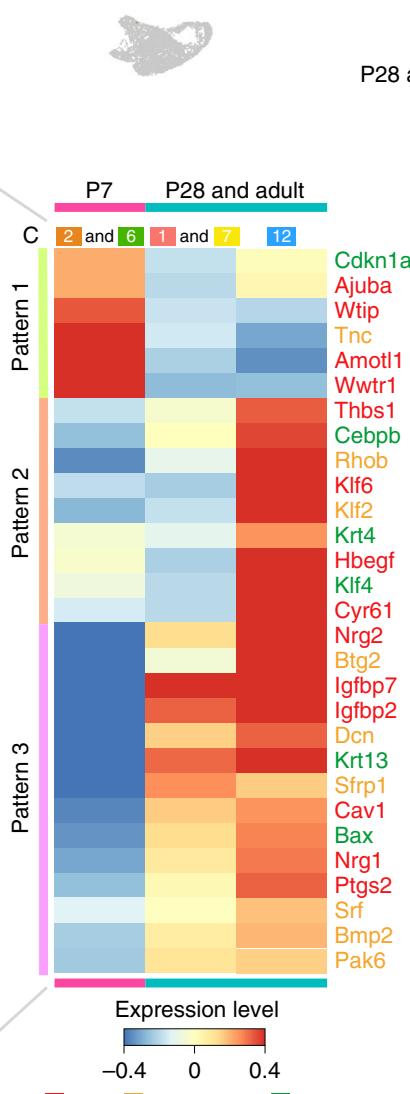

$\square$ YAP Mechanics $\square$ KLF4 profile strengthens histological observations suggesting that $\mathrm{KLF}^{+}$basal cells represent a committed population established at the onset of homeostasis.

C

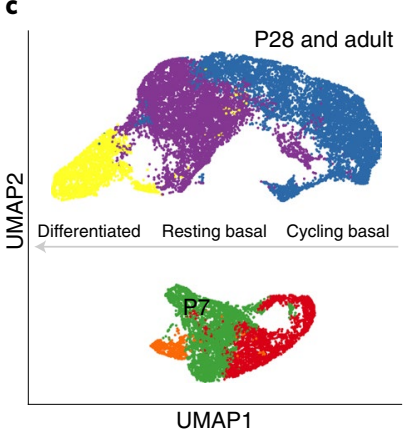

P7: CB RB D P28 and adult: $\triangle \mathrm{CB} \quad \mathrm{RB} \mathrm{D}$
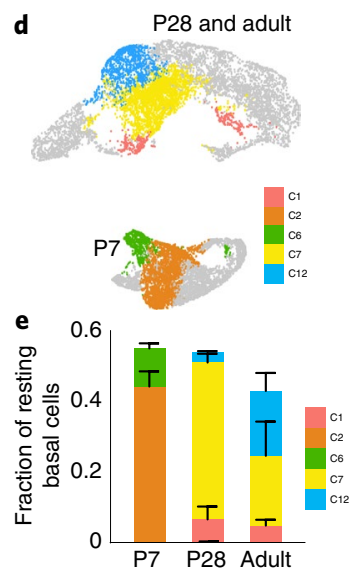

$\mathbf{g}$
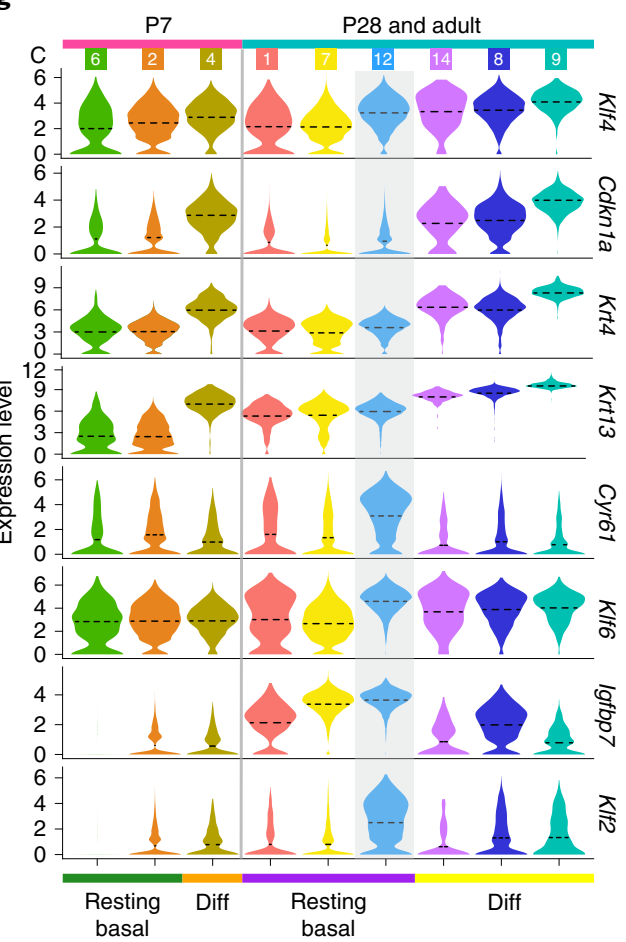

h

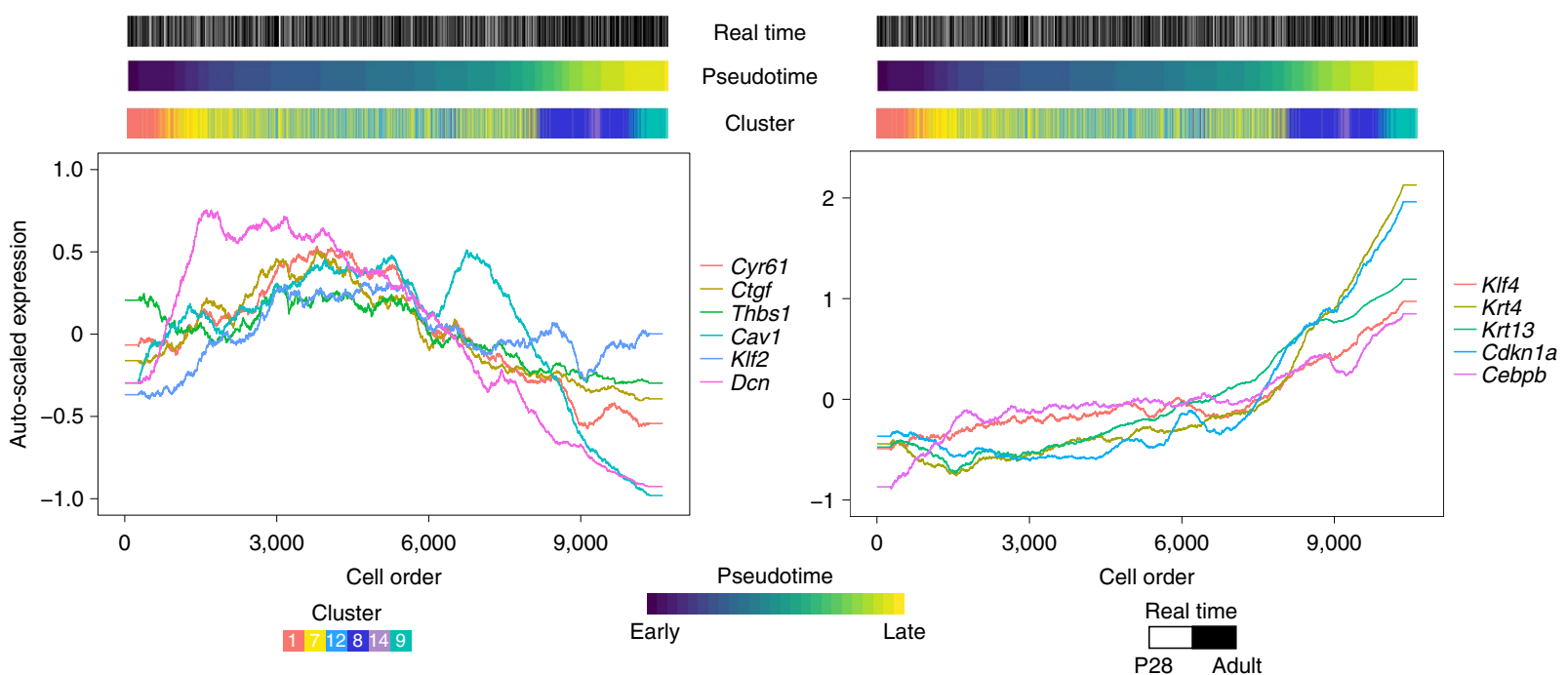



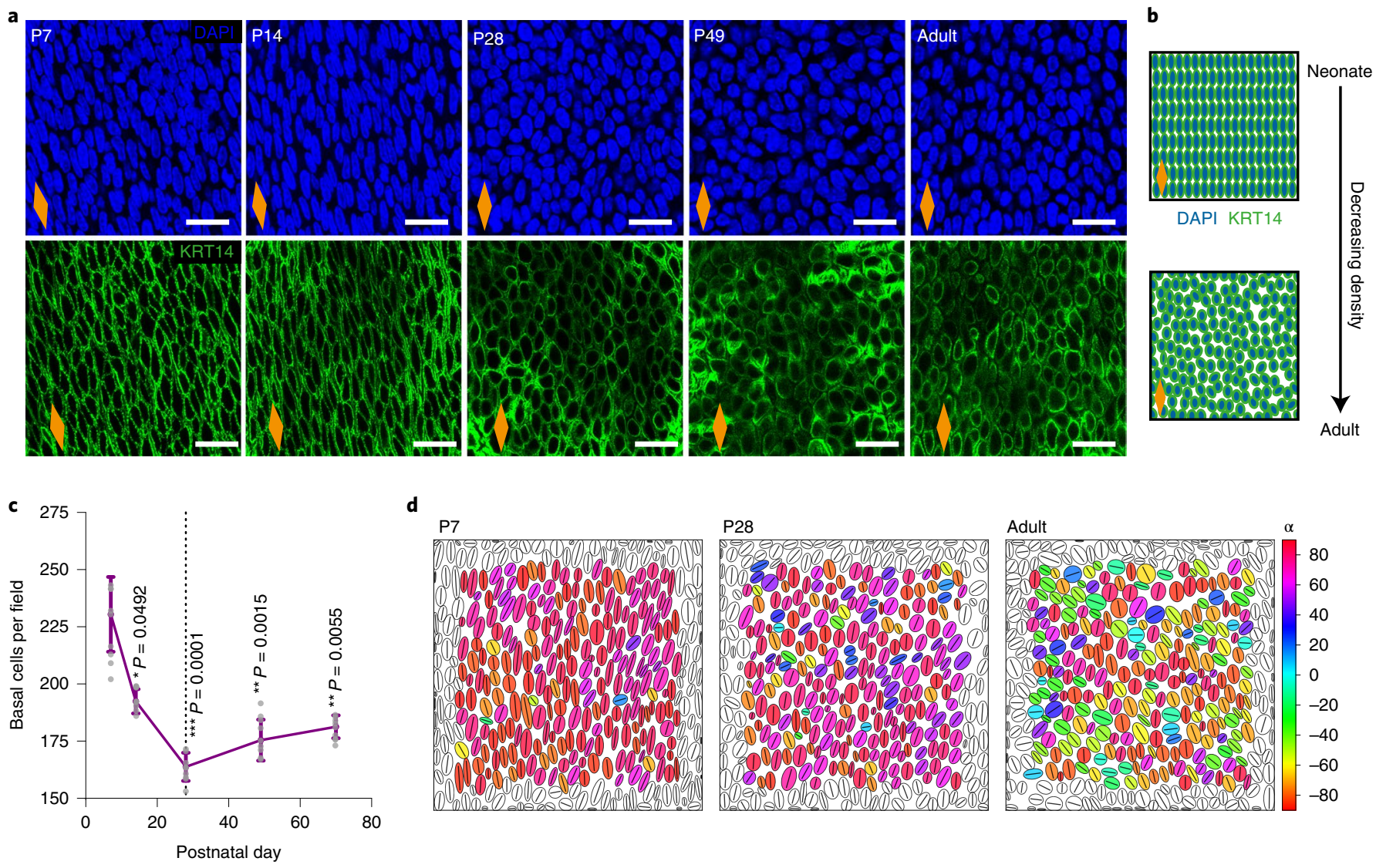

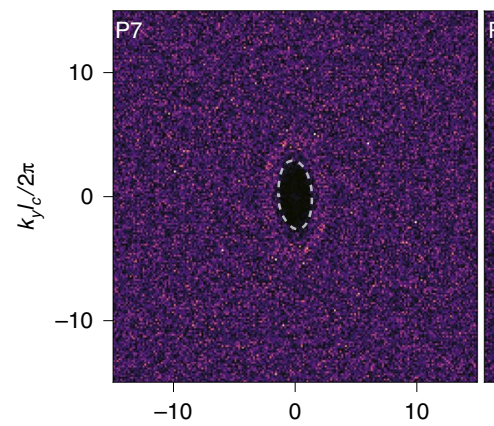

d

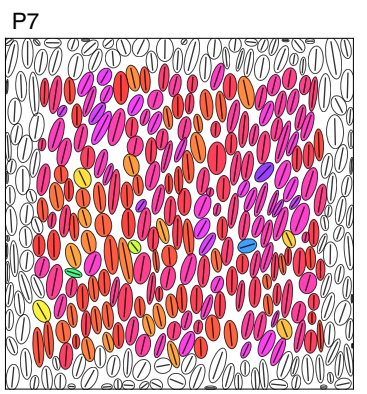

P28

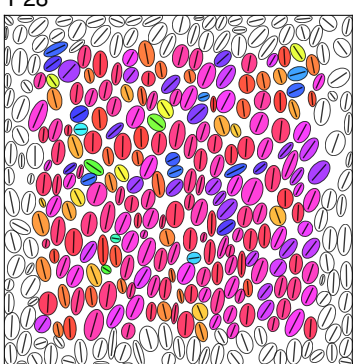

Adult

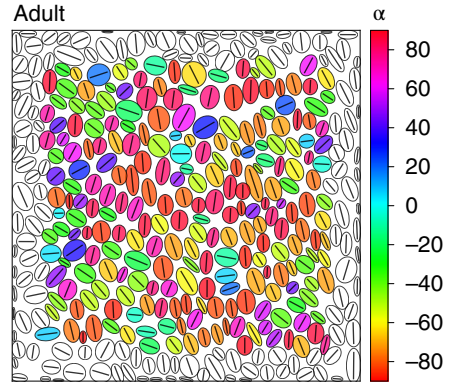

Fig. 4 | Homeostatic transition coincides with tissue spatial reorganization. a, Typical basal views of confocal z-stacks showing changes in cell shape, alignment and density in oesophageal epithelial wholemounts from different developmental stages. Blue and green represent DAPI and KRT14, respectively. Scale bars, $20 \mu \mathrm{m}$. b, Schematic exemplifying the changes seen in a. c, Quantification of basal cell density. The data are expressed as means \pm s.e.m. $(n=3$ mice). The vertical dashed line indicates P28. d. Cell shape anisotropy tensor, represented as an ellipse calculated from the nuclear centroid position of each basal cell. The long axis of each ellipse is proportional to the dominant eigenvalue of the tensor. Orientation is colour coded ( $\alpha$, angle to horizontal line in degrees angle degrees). The results from one representative experiment are shown ( $n=3$ mice). e, Bidimensional structure factor $S(k)$ as a function of wave vector $\left(k_{x}, k_{y}\right)$ scaled by the average basal cell separation, $I_{c}$ (see Supplementary Note 1). Changes in the dashed white outline (from ellipse to circle) depict a transition from anisotropic to isotropic spatial cell distribution over time ( $n=3$ mice). $\mathbf{f}$, Box and whisker plots of the nematic order parameter, which is indicative of the orientational order of cells in the tissue. The box central lines and edges show median values and quartiles, respectively, and the whiskers represent minimum and maximum values ( $n=3$ mice). Nuclei at the edge of the image frame in $\mathbf{d}$ and $\mathbf{e}$ were discarded to avoid confounding effects from partially captured cells. All data were derived from wild-type C57BL/6J mice. Statistical significance in $\mathbf{c}$ and $\mathbf{f}$ was determined by one-way ANOVA with Tukey's multiple comparisons test $\left({ }^{\star} P<0.05,{ }^{\star \star} P<0.01,{ }^{\star \star \star} P<0.001,{ }^{\star \star \star \star} P<0.0001\right.$ relative to $\left.\mathrm{P} 7\right)$. The orange diamonds in $\mathbf{a}$ and $\mathbf{b}$ depict the longitudinal orientation of the oesophagus (as outlined in Extended Data Fig. 1a). See also Supplementary Note 1 and Extended Data Fig. 5.

Another feature defining resting basal cells at later time points was the differential expression of genes associated with a response to mechanical stimuli and YAP mechanosensing (Fig. 3f) (2-65 $^{62}$. Interestingly, these genes were enriched at later time points (P28 and adult) and more particularly in the cluster containing the
Klf $4^{\text {high }}$ population (C12; Fig. 3f,g). More specifically, P28 and adult samples were marked by an increase in Cyr61 expression (a direct downstream target of YAP in mouse keratinocytes), as well as other genes modulated by YAP, including (among others) Thbs1, Nrg1, Nrg2, Klf6, Hbegf, Ptgs2, Igfbp2 and Igfbp7 (Fig. 3f,g) ${ }^{66-71}$. Cav1 
(a key cellular mechanoregulator also known to positively control YAP in response to substrate stiffness) showed increased expression at the P28 and adult stages ${ }^{66,72,73}$. Additionally, the expression of Klf2, a shear stress-responsive transcription factor known to be critical for heart valve development, was also significantly increased at later time points ${ }^{74,75}$ (Fig. 3f,g). Accordingly, Amotl1, Wtip and $A j u b a$, which have been proposed to act as negative regulators of the mechanosensing pathway Hippo ${ }^{76,77}$, showed increased expression at P7 compared with later time points (Fig. 3f).

Given the concurrence of genes associated with KLF4 and tissue mechanics, we then focused on the dynamic pattern of gene expression to unveil any clues suggesting potential co-regulation. For this, we performed pseudotime analysis, inferring the differentiation path within the resting basal population at early (P7; Extended Data Fig. 4f) and later time points (P28 and adult; Fig. 3h; Methods). Next, we examined gene expression along the pseudotime trajectory. At later time points, YAP-associated genes, including upstream regulators (Cav1) and direct targets (Cyr61, Ctgf and Thbs1), presented a distinctive pattern of expression that peaked in $\mathrm{C} 12$, decreasing thereafter as differentiation became established (C14, C8 and C9; Fig. 3g,h). Other mechanics-related genes, such as Dcn and Klf2, presented a similar trend, with Dcn showing a much wider peak of expression that spanned C7 and C12. Klf4 expression was also higher in $\mathrm{C} 12$, but as anticipated for a differentiation marker, its expression increased progressively throughout the differentiation trajectory (C14, C8 and C9; Fig. 3g,h). Accordingly, KLF4 downstream targets (Krt4, Krt13, Cdkn1a and Cebpb) showed a subsequent increase in the late phases of the pseudotime trajectory, representing the most differentiated stages (Fig. 3g,h). These results point to a link between a response to mechanical stimuli and the expression of KLF4 in the basal layer of the oesophageal epithelium at late time points; a similar association could not be made at P7 (Extended Data Fig. 4f).

Our scRNA-seq analysis highlights a clear shift in cell state through postnatal development, from being highly morphogenic at P7 to a more mature state at P28 and adult. Moreover, the uncovered transcriptional signature suggests a potential link between changes in tissue mechanics and a transition towards commitment in basal progenitor cells.

Differential growth leads to build-up in mechanical strain. The biomechanical scRNA-seq signatures, together with considerable changes in basal cell morphology and density (Fig. $4 \mathrm{a}-\mathrm{c}$ and Extended Data Fig. 5a,b), prompted us to look deeper into the architecture of the basal oesophageal epithelium. We developed a deep-learning image segmentation analysis pipeline (Extended Data Fig. $5 \mathrm{c}-\mathrm{e})$ to accurately quantify postnatal changes in cell shape, orientation and spatial organization. Our analysis shows that the anisotropic pattern and long-range orientational alignment observed in basal cells at early time points becomes less apparent from P28 onwards (Fig. 4d-f and Extended Data Fig. 5f-i), reflecting temporal changes in the physical properties of the tissue (Supplementary Note 1).

So far, our data hint to a possible link between changes in tissue mechanics and the transition towards homeostasis. We speculated that a potential source of mechanical stress may originate from growth anisotropy between the oesophageal epithelium, neighbouring tissues and/or the whole body of the animal. To explore this scenario, we measured the length of the oesophagus postmortem (in situ), within the animal and after tissue dissection (ex vivo) at different postnatal time points (Fig. 5a,b and Extended Data Fig. 6a). The increase in length relative to P7 (in situ and ex vivo) was compared with that of the body length (Fig. 5c). Interestingly, we observed a discrepancy in oesophageal length before and after dissection, with the ex vivo oesophagus becoming significantly shorter from P28 to adult. We established that the differential growth of the developing oesophagus, under normal physiological conditions, results in the build-up of a progressive strain, estimated to be between 20 and 40\% from P28 to adult (Fig. 5d, Extended Data Fig. 6b,c and Supplementary Note 1). This growth pattern was not observed in the stomach, which showed a continuous increase in size and proliferative activity during the final postnatal stages (Extended Data Fig. 6d-g). Interestingly, this mechanical transition coincided with an active remodelling of the extracellular matrix, as evidenced by temporal changes in second harmonic generation (SHG; Extended Data Fig. 6h,i and Supplementary Note 1). We therefore conclude that the oesophageal epithelium undergoes a mechanically mediated transition, from being densely packed at earlier time points (when rapid proliferation promotes cell crowding; Fig. 4c) to becoming more loosely packed and stretched upon homeostasis (Fig. 5c,d).

To test whether the observed changes in the mechanical properties of the oesophagus stimulated a switch in tension and cytoskeleton remodelling of epithelial cells, we labelled the oesophageal epithelium for F-actin and phosphorylated myosin light chain II (pMLC2), a marker of cortical actomyosin contractility. Both presented a similar profile, increasing their signal as tissue matured (Fig. $5 \mathrm{e}-\mathrm{j}$ and Supplementary Note 1). Next, we interrogated the cytoplasmic-to-nuclear translocation of the transcription factor YAP, an effector of the mechanosensing pathway $\mathrm{Hippo}^{78}$. YAP

Fig. 5 | Differential growth generates longitudinal tissue strain that is sensed at the cellular level. a, Method for oesophageal length measurements $(I)$ in situ and ex vivo. b, In situ and immediate ex vivo images of oesophageal tubes captured in P7 and adult mice. The dashed lines delineate the oesophageal tube. c, Percentage increase in oesophageal length (in situ and ex vivo) compared with body length (excluding tail) throughout postnatal development (P7 set to 100\%). The vertical dashed line indicates P28. The data are expressed as means \pm s.e.m. Each time point was analysed by two-tailed unpaired $t$-test $\left({ }^{\star \star} P<0.01,{ }^{\star \star \star} P<0.001,{ }^{\star \star \star \star} P<0.0001\right.$ grey and blue labels indicate the difference between body and ex vivo and between body and in vivo, respectively; $n=6$ mice). $\mathbf{d}$, Longitudinal tissue strain in vivo, represented as percentage. The data are expressed as means \pm s.e. $\mathrm{m}$. ( $n=6$ mice). e,f, Schematics representing changes in F-actin (e; grey) and pMLC2 levels (f; green) in oesophageal epithelial cells throughout postnatal development. $\mathbf{g}$, Basal views of typical oesophageal epithelial wholemounts showing phalloidin staining for F-actin at the indicated time points. Blue and greyscale represent DAPI and phalloidin, respectively. $\mathbf{h}$, Representative side views of tissue sections showing pMLC2 staining at the indicated time points. Blue and green represent DAPI and pMLC2, respectively. Dotted lines, basement membrane; ep, epithelium; strm, stroma. i,j, Quantification of basal F-actin (i) and pMLC2 staining (j) (see Methods). Central lines and box edges show median values and quartiles, respectively, and whiskers represent minimum and maximum values ( $n=3$ mice in $\mathbf{i} ; n=4$ mice in $\mathbf{j}$ ). $\mathbf{k}$, Schematic of the changes in YAP (green) localization during postnatal development. $P$ denotes YAP phosphorylation. I, Basal views of representative oesophageal epithelial wholemounts, showing progressive translocation of YAP to the nucleus as the tissue matures. Blue, green and greyscale represent DAPI, YAP and $\beta$-catenin ( $\beta$-cat), respectively. $\mathbf{m}$, Quantification of basal nuclear (Nuc) and cytoplasmic (Cyto) YAP staining (see Methods). A total of 60 cells per time point were measured from three animals. The localization pattern shows the average intensity for one representative animal per time point. Full quantification is provided in Extended Data Fig. $6 \mathrm{j}, \mathrm{k}$. Scale bars, $1 \mathrm{~cm}$ (b), $20 \mu \mathrm{m}\left(\mathbf{g}\right.$ and $\mathbf{I}$ ) and $10 \mu \mathrm{m}$ (h). Statistical significance in $\mathbf{d}, \mathbf{i}$ and $\mathbf{j}$ was determined by one-way ANOVA with Tukey's multiple comparisons test ( ${ }^{\star \star} P<0.01$, ${ }^{\star \star \star} P<0.001,{ }^{\star \star \star \star} P<0.0001$ relative to Adult). The orange diamonds represent the longitudinal orientation of the oesophagus (Extended Data Fig. 1a). Parts of a were drawn using and/or adapting diagrams from Servier Medical Art. See also Supplementary Note 1 and Extended Data Fig. 6. 
nuclear localization is associated with increased space availability, cell spreading and subsequent cytoskeletal reorganization. As predicted by the increase in tensile strain observed in the oesophageal epithelium, YAP was found to translocate from its cytoplasmic localization at P7 to the nucleus in adult animals, with an intermediate state at P28 reflecting a mechanosensing transition (Fig. $5 \mathrm{k}-\mathrm{m}$ and Extended Data Fig. 6j-1). These data are indicative of basal progenitors sensing the physiological changes in strain and space availability experienced by the tissue.

We conclude that the differential growth of the oesophagus results in the build-up of a longitudinal tensile strain as the oesophageal epithelium transitions towards homeostasis. Changes in the cellular response to mechanical stimuli, which were initially evidenced at the transcriptional level, were further reinforced by the cytoplasmic-to-nuclear translocation of the Hippo effector YAP.

YAP promotes basal commitment through KLF4. The experiments above suggest a temporal correlation between YAP and KLF4

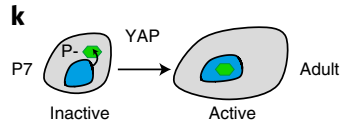

g
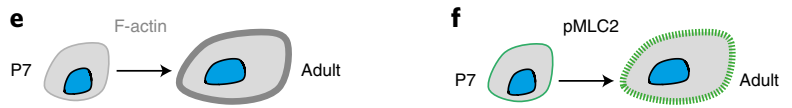

I

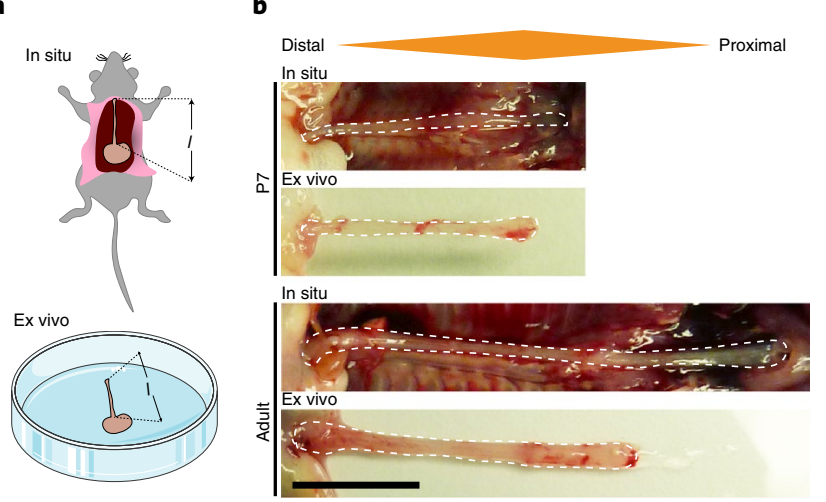

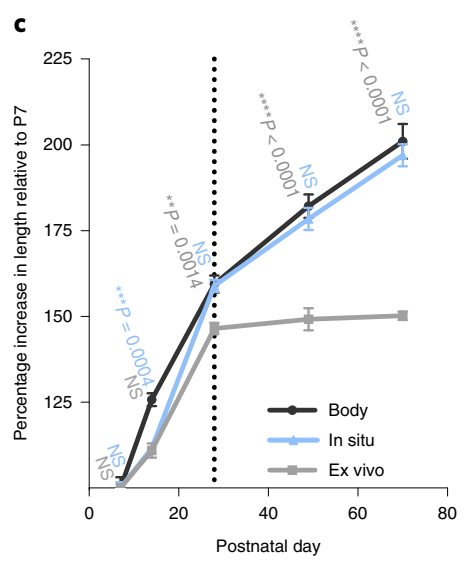

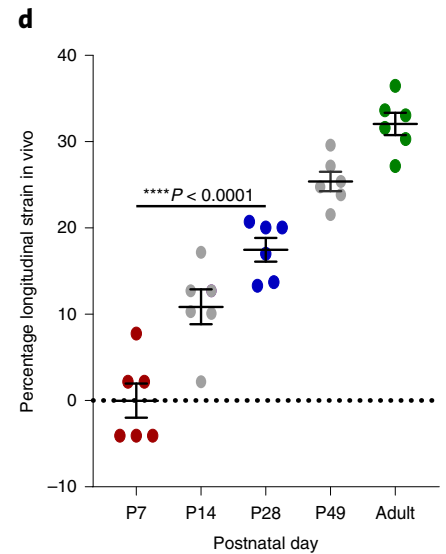

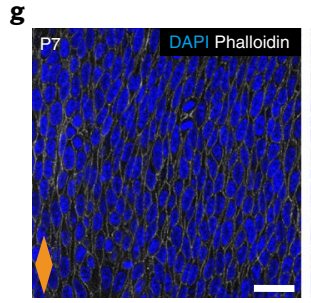
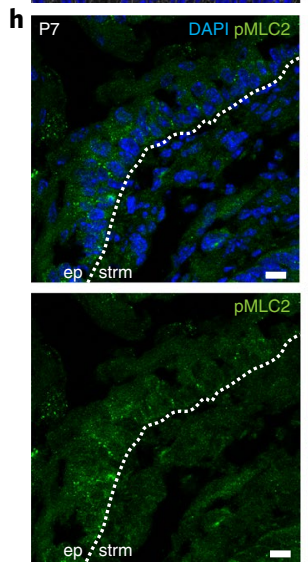

i

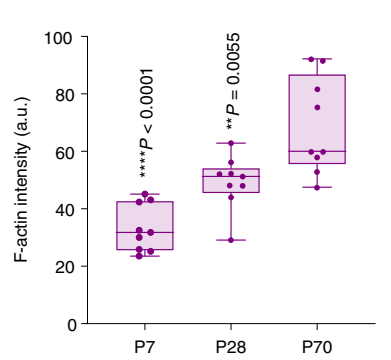

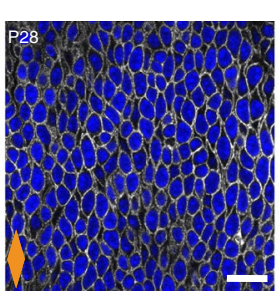
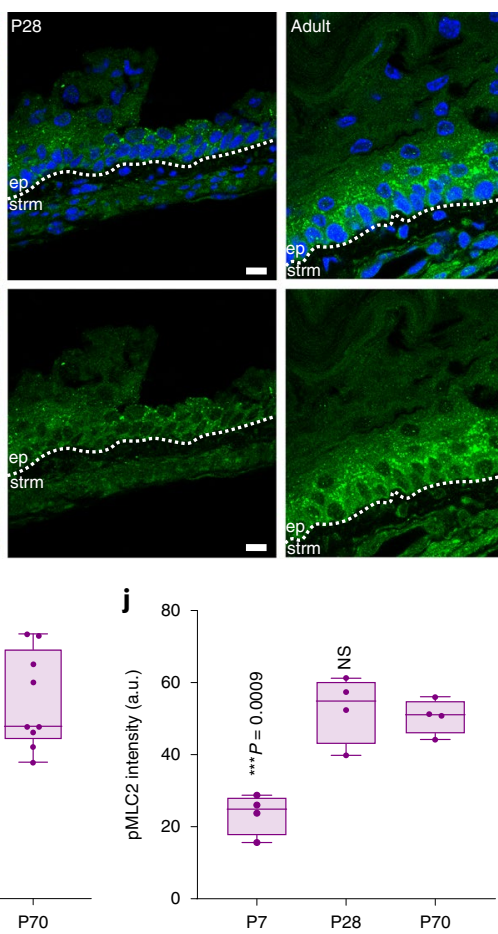
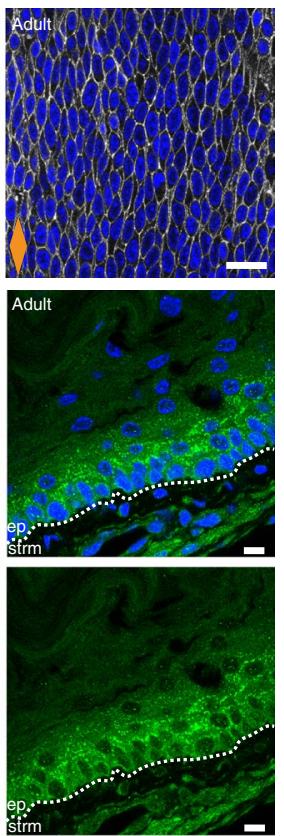
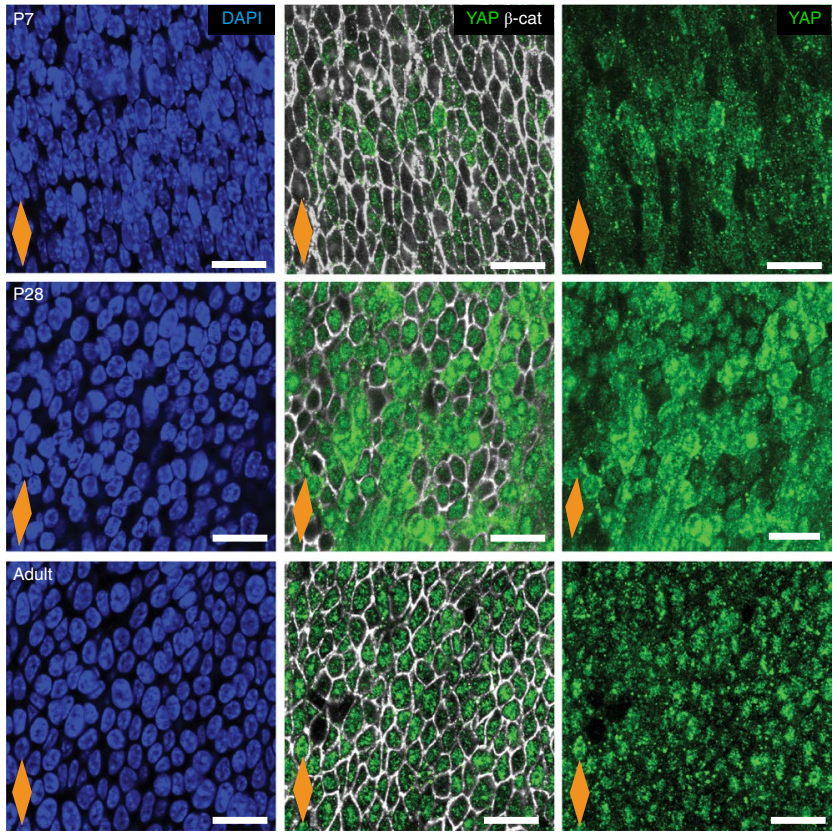

m
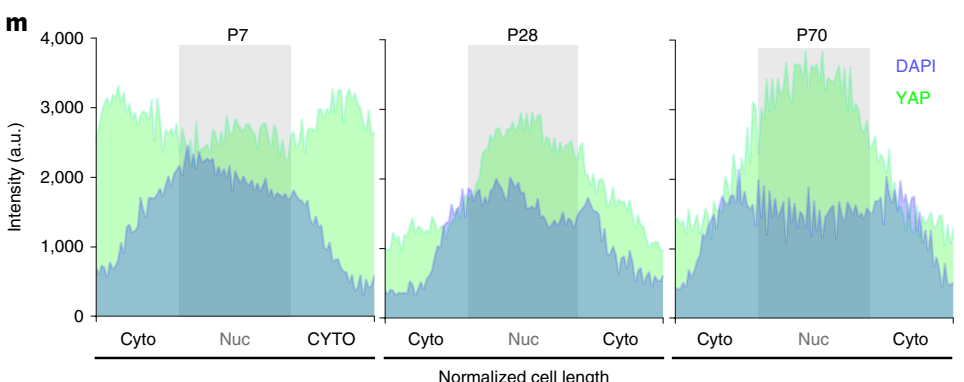

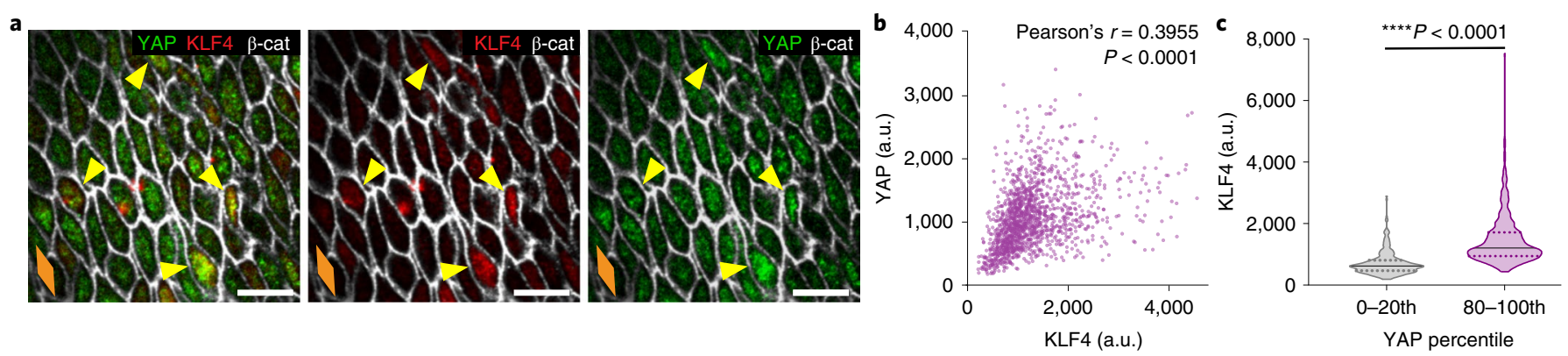

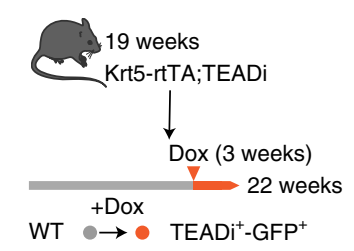

f

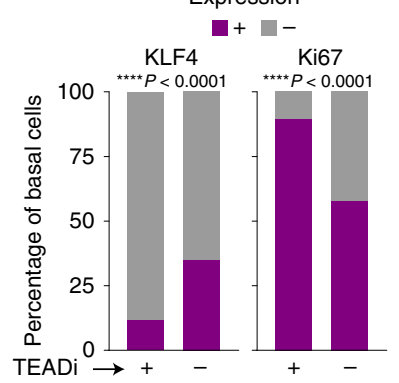

j

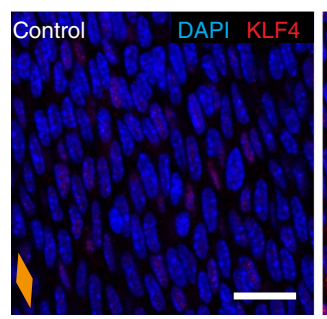

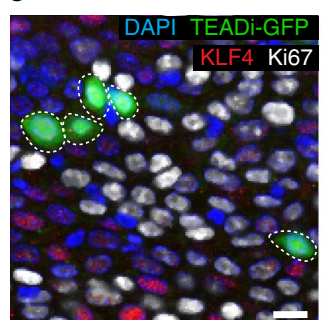

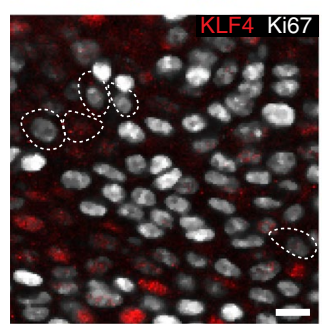

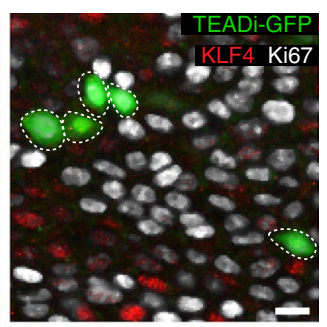

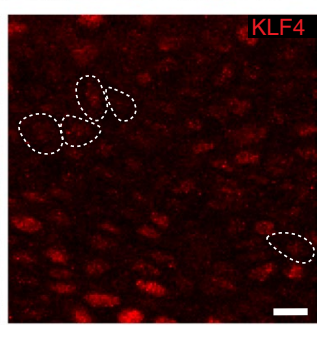

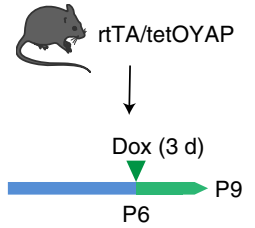

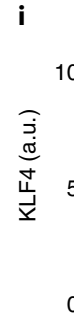

h
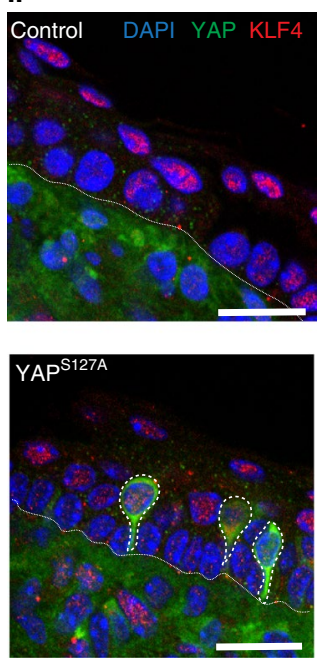

Control YAP $^{\text {S127A }}$

k
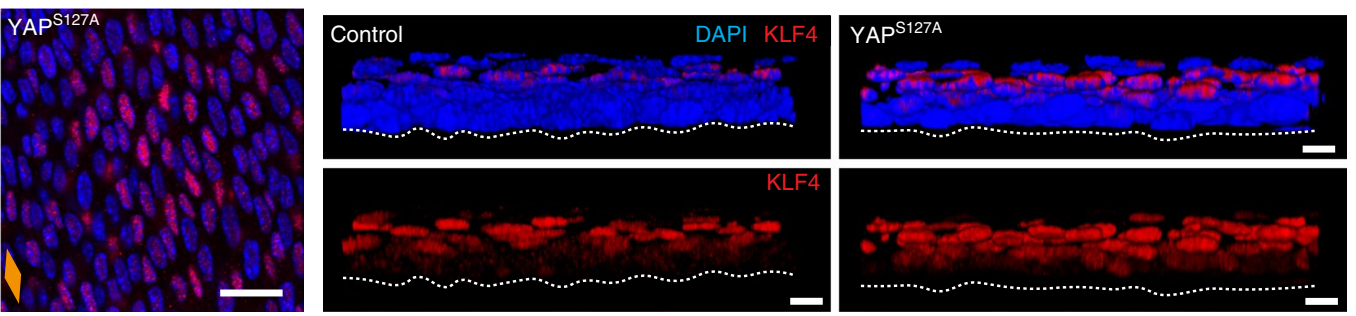

Fig. 6 | YAP nuclear localization promotes increased levels of basal commitment through KLF4. a, Confocal basal views of typical adult oesophageal epithelium from wild-type C57BL/6J mice. The yellow arrowheads indicate co-localization of KLF4 (high) and YAP (high) staining. b, Correlation plot for KLF4 and YAP intensities in the basal layer of the adult oesophageal epithelium in $\mathbf{a}(n=3$ mice). Data correlation performed using Pearson's correlation coefficient $(r)$. c, Quantification of KLF4 intensity (a.u.) within the lowest and highest YAP-expressing cells (0-20th and 80-100th percentiles, respectively). $n=296$ cells per percentile from three mice. $\mathbf{d}$, In vivo protocol. Adult Krt5-rtTA;TEADi mice were treated with Dox for 3 weeks to block YAP- and TAZ-induced TEAD activity in basal cells. TEADi-expressing cells are marked by GFP expression (TEADi+). WT, wild type. e, Representative basal views of the oesophageal epithelial wholemounts from $\mathbf{d}$. TEADi-expressing cells are marked by GFP expression. The dashed circles indicate GFP+ cells. f, Percentage of $\mathrm{KLF}^{+/-}$and $\mathrm{Ki}^{6} 7^{+/-}$cells in TEADi+/- basal cells from $\mathbf{d}$ (as marked by GFP). A total of $800 \mathrm{TEADi}^{+/-}$basal cells were quantified from 72 fields from two mice. Statistical significance was determined by one-sided Chi-squared test $\left.{ }^{\star \star \star \star} P<0.0001\right)$. g, In vivo protocol. P6 rtTA/ tetOYAP pups were treated with Dox for $3 \mathrm{~d}$ to induce an active form of YAP (p.Ser127Ala) and culled at P9. $\mathbf{h}$, Staining of optimal cutting temperature compound-embedded (OCT) oesophageal epithelial cryosections ( $10 \mu \mathrm{m}$ thick) of YAP-activated mice from $\mathbf{g}$. Nuclear KLF4 expression co-localized with $\mathrm{YAP}^{+}$cells. The dashed white lines denote $\mathrm{YAP}^{+}$cells. $\mathbf{i}$, KLF4 intensity quantification (a.u.) in control and YAP-overexpressing mice, as shown in $\mathbf{j}$ ( $n=1,362-1,779$ cells from three animals). $\mathbf{j}$, Representative basal views of oesophageal epithelial wholemounts from $\mathbf{g}$. $\mathbf{k}$, Representative 3D-rendered side views of oesophageal epithelial wholemounts from $\mathbf{g}$, showing the expanded KLF4 suprabasal compartment in rtTA/tetOYAP mice compared with controls. Scale bars, $10 \mu \mathrm{m}(\mathbf{a}, \mathbf{e}$ and $\mathbf{k})$ and $20 \mu \mathrm{m}$ (h and $\mathbf{j})$. Blue staining represents DAPI, green represents YAP (a and $\mathbf{h}$ ) or TEADi-GFP (e), red represents KLF4 $(\mathbf{a}, \mathbf{e}, \mathbf{h}, \mathbf{j}$ and $\mathbf{k})$ and greyscale represents $\beta$-catenin (a) or Ki67 (e). Dotted line, basement membrane (h,k). Violin plots (c,i) show median (solid line) and quartiles (dotted lines). The data from $\mathbf{c}$ and $\mathbf{i}$ were analysed by two-tailed unpaired $t$-test ( ${ }^{\star \star \star \star} P<0.0001$ ). The orange diamonds depict the longitudinal orientation of the oesophagus (as outlined in Extended Data Fig. 1a). Parts of $\mathbf{d}$ and $\mathbf{g}$ were drawn using and/or adapting diagrams from Servier Medical Art. See also Supplementary Note 1.

expression patterns, with both transcription factors becoming localized to the nucleus of basal cells as the tissue transitions towards homeostasis (Fig. 2 and Fig. 5l,m). To explore the existence of a potential link between the two, we used three different approaches.
First, we co-stained and correlated the levels of both proteins in the nucleus of adult basal cells (Fig. 6a-c). Second, we looked at KLF4 expression upon interfering with YAP signalling in adult mice. For this, we used an inducible mouse line $\left(K r t 5\right.$-rtTA; TEADi-GFP $\left.{ }^{79}\right)$, 
where genetic induction results in blockage of YAP- and tafazzin (TAZ)-induced TEAD (transcriptional enhancer factor) activity in the basal cell compartment, as detected by the TEADi-GFP (green fluorescent protein) reporter (Fig. 6d-f). Third, we investigated the impact of YAP overactivation in the early postnatal oesophageal epithelium (P6-P8), when basal cells are still negative for KLF4 (Fig. 2a,b,g), using the doxycycline (Dox)-inducible mouse strain Rosa26-rtTA; tetO-YAP ${ }^{\mathrm{S} 127 \mathrm{~A}}$ (rtTA/tetOYAP), which has a p.Ser127Ala substitution in YAP (Fig. 6g-k). Collectively, these experiments showed a correlation between YAP and KLF4 expression, and demonstrated that YAP nuclear activity promotes KLF4 expression in the basal cell compartment, balancing progenitor cell behaviour through commitment (Supplementary Note 1).

Changes in tissue mechanics influence basal KLF4 expression. Having identified an increase in tensile strain throughout postnatal development, we next interrogated whether the mechanical stress imposed at the whole-organ level was sensed at the cellular level. To answer this question, we used a whole-organ in vitro approach utilising a 3D-printed stretching device ${ }^{80}$ (Fig. 7a,b and Extended Data Fig. 7a) that allows for controlled uniaxial tissue stretching (Methods). Adult oesophagi were stretched along their longitudinal axis at a static strain of $40 \%$, representing the physiological levels measured in the adult oesophagus (Fig. 5d, Extended Data Fig. $6 \mathrm{c}$ and Methods). As a result, there was an increase in basal cell area (Extended Data Fig. 7b,c) that could be rescued by blebbistatin (BLEBB) treatment, which inhibits myosin II motor activity, disrupting tissue tension and cell junction stability (Extended Data Fig. 7b-d; 40\% stretch). Accordingly, BLEBB led to a marked cellular disorganization. These results suggest that basal cells bear part of the mechanical load experienced upon whole-organ stretching (Supplementary Note 1).

Next, we investigated the relevance of mechanical stress in driving the transition to homeostasis. Oesophageal samples from P7, P28 and adult animals were stretched to adult physiological levels (40\%). The expression of KLF4 was analysed as a proxy to identify changes in cell commitment in the basal layer. Reassuringly, the unstretched in vitro controls exhibited a similar KLF4 expression profile to that observed in vivo (Fig. 7c (top panels) versus Fig. 2b). Moreover, KLF4 expression remained unchanged in adult stretched samples, confirming that the strain was within the physiological range (Fig. 7c,d). P7 and P28 samples, instead, showed a significant increase in KLF4 basal levels that reached control adult values upon stretching (Fig. 7c,d). These data suggest that physiological levels of tensile stress promote KLF4 expression in the basal compartment. Further experiments exposing P7 samples to a range of tensile strains revealed that changes in basal KLF4 expression respond to a strain threshold between 20 and 40\% (Fig. 7e), matching the levels found at the transition between P28 and adult (Fig. 5d, Extended Data Fig. $6 c$ and Methods). Accordingly, the proliferative activity of the oesophagus at P7 was reduced at $40 \%$ strain (Extended Data Fig. $7 \mathrm{e}-\mathrm{h}$ ). Contrary to physiological levels of strain, stretching by $100 \%$ promoted an increase in proliferative activity, in line with previous studies on overstretched tissues ${ }^{81}$. These results support the notion of a biomechanical switch operating during the homeostatic transition of the oesophageal epithelium that dictates a shift in cell behaviour.

Remarkably, KLF4 levels in adult unstretched controls closely resembled those of in vivo counterparts (Fig. 7c). This suggests that, while an increase in strain is sufficient to activate KLF4 expression in basal cells, it does not seem necessary to maintain it in adult cells, where suprabasal keratinization provides the means to retain tissue architecture to a certain extent (Extended Data Figs. 1j,k and $7 \mathrm{i}-\mathrm{k})^{82,83}$.

Supporting the idea that basal KLF4 expression responds to changes in tissue mechanics, in vitro treatment with BLEBB to lower the tissue tension (Extended Data Fig. 7l), by disrupting actomyosin contractility, led to a reduction in KLF4 expression in the adult oesophageal epithelium (Fig. 7f-h), although no impact on cell proliferation was observed (Extended Data Fig. 7l,m). Further mechanistic insight was achieved by treating stretched P7 oesophagi with verteporfin, an established pharmacological inhibitor of YAP ${ }^{84,85}$. Verteporfin rescued the strain-induced KLF4 response (Fig. 7i-k), demonstrating that the mechanical regulation of KLF4 expression is mediated by YAP.

Taken together, our results reveal that the longitudinal tensile strain, generated by the anisotropic growth of the oesophagus during postnatal development, must reach a critical threshold in order to affect cell behaviour. Once this threshold is reached, basal KLF4 expression increases, modulated via YAP. This results in progenitor cells tilting their balance towards commitment, which ensures the establishment of tissue homeostasis (Fig. 7l).

\section{Discussion}

Here, we report that the physiological strain emerging during normal postnatal development of the oesophagus controls its maturation, temporally guided by a mechanical threshold. In particular, we show that the differential growth of the oesophagus relative to the entire body generates a level of mechanical stretch that influences epithelial cell fate and promotes the transition towards homeostasis. Mechanistically, our data indicate that physiological stretching of the oesophagus results in nuclear re-localization of the YAP mechanosensor, which in turn promotes the emergence of a $\mathrm{KLF}^{+}$committed population in the basal progenitor

Fig. 7 | Changes in tissue mechanics influence basal KLF4 expression. a, In vitro protocol. Oesophageal tubes were exposed to a $40 \%$ stretch using a 3D-printed stretcher and kept in vitro as whole-organ cultures for $48 \mathrm{~h}$. b, Left: 3D model of the stretching device. Right: Example of the device in use. c, Confocal basal views of stretched and unstreched control samples after $48 \mathrm{~h}$. $\mathbf{d}$, Basal quantification of $\mathrm{KLF}^{+}{ }^{+}$cells, expressed as percentage of $\mathrm{DAPI}^{+}$basal cells from $\mathbf{c}\left(n=5\right.$ mice for P7 and P28; $n=6$ mice for adults). e, Threshold response. Basal quantification of KLF4 ${ }^{+}$cells in P7 whole-organ cultures after exposure to 20,40 or $100 \%$ longitudinal stretch versus unstreched control conditions for $24 \mathrm{~h}$ (as in $\mathbf{a}$ ). $n=3$ mice. $\mathbf{f}$, In vitro protocol. Adult epithelial-stromal composites were kept in vitro and treated with $25 \mu \mathrm{M}$ BLEBB for $48 \mathrm{~h}$ and EdU for the final $2 \mathrm{~h}$. $\mathbf{g}$, Confocal basal views after a 48-h BLEBB treatment in vitro (f). h, Percentage of KLF4+ basal cells relative to DAPI+ basal cells from $\mathbf{f}$ ( $n=3$ mice). $\mathbf{i}$, In vitro protocol. Oesophagi were exposed to $40 \%$ stretch and treated with $1 \mu \mathrm{M}$ DMSO/verteporfin (VP) for $24 \mathrm{~h}$. j, Confocal basal views after $24-\mathrm{h}$ VP treatment in vitro (i). k, KLF4 intensity quantification (a.u.) in oesophageal epithelial basal cells from $\mathbf{i}(n=794-1,398$ cells from three animals per condition). I, Suggested model. The physiological stretching experienced by the postnatal oesophagus promotes the expression of KLF4 in the basal progenitor cell compartment in a YAP-dependent manner. The onset of basal KLF4 expression marks the emergence of an early committed population, which balances proliferation and defines the transition towards adult homeostasis. Scale bars, $1 \mathrm{~cm}$ (b), $5 \mathrm{~mm}$ (inset in $\mathbf{b}$ ) and $20 \mu \mathrm{m}$ (c,g and j). Blue, red and greyscale stainings represent DAPI, KLF4 and E-cadherin, respectively. The data in $\mathbf{d , e , h}$ and $\mathbf{k}$ are expressed as means \pm s.d. Statistical significance in $\mathbf{e}$ and $\mathbf{k}$ was determined by one-way ANOVA with Tukey's multiple comparisons test $\left({ }^{\star} P<0.05,{ }^{\star \star \star \star} p<0.0001\right)$. The results in e are relative to the control. Statistical significance in $\mathbf{d}$ and $\mathbf{h}$ was determined by two-tailed unpaired $t$-test $\left({ }^{\star} P<0.05,{ }^{\star \star \star \star} p<0.0001\right)$. Individual points show individual measurements. Greyscale indicates values from each of three mice. Orange diamonds represent the longitudinal orientation of the oesophagus (Extended Data Fig. 1a). Parts of a,f,i and $\mathbf{I}$ were drawn using and/or adapting diagrams from Servier Medical Art. See also Extended Data Fig. 7. 
compartment. The onset of basal cell commitment towards differentiation balances proliferation and ultimately defines the transition to adult homeostasis.

a
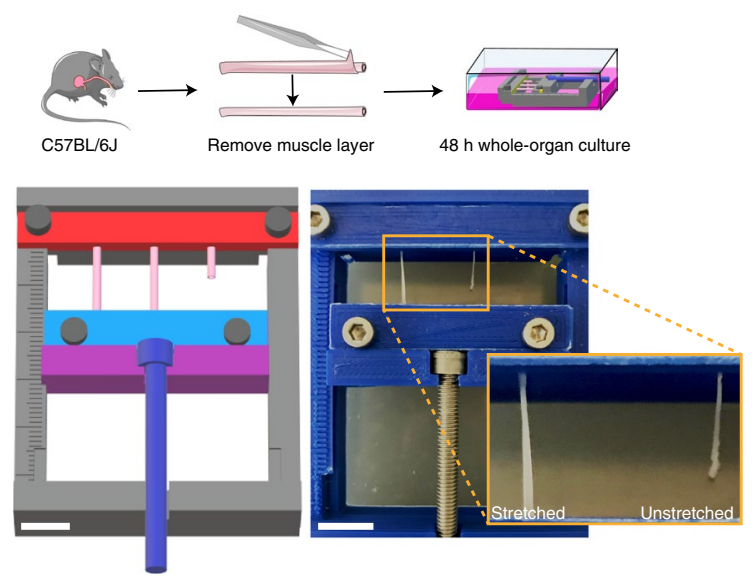

d

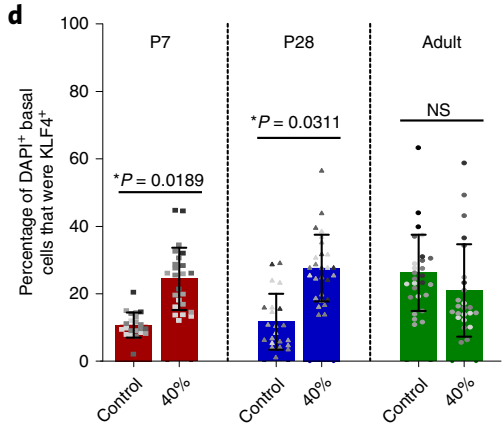

f

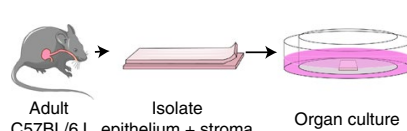

g
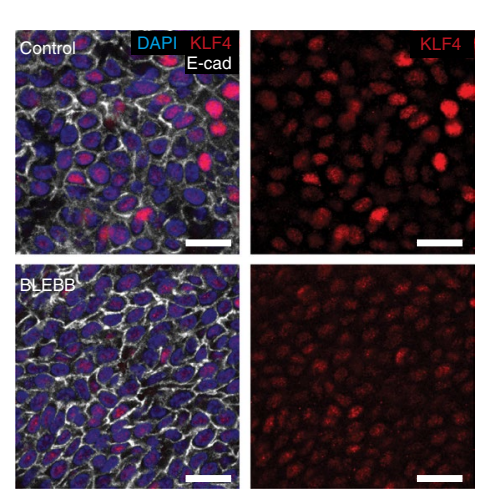
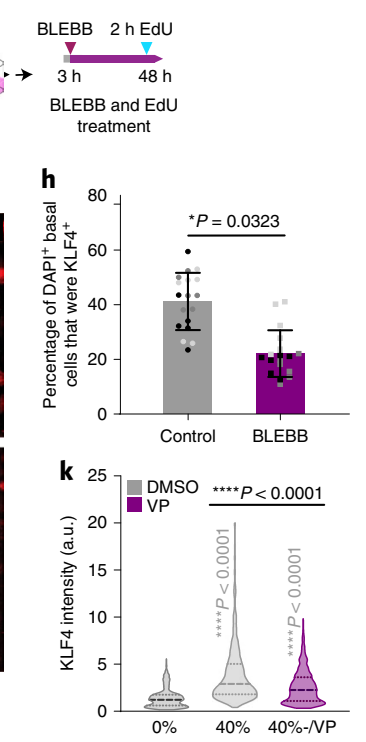

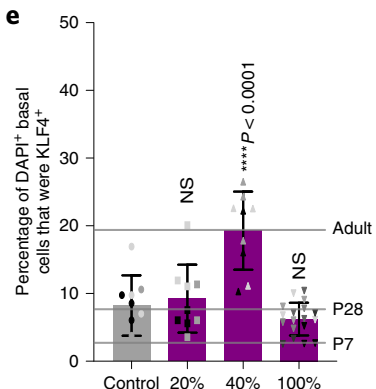

Tissue morphogenesis has long been known to be orchestrated by finely balanced gradients of morphogens. More recent studies in a growing number of developmental systems are starting to

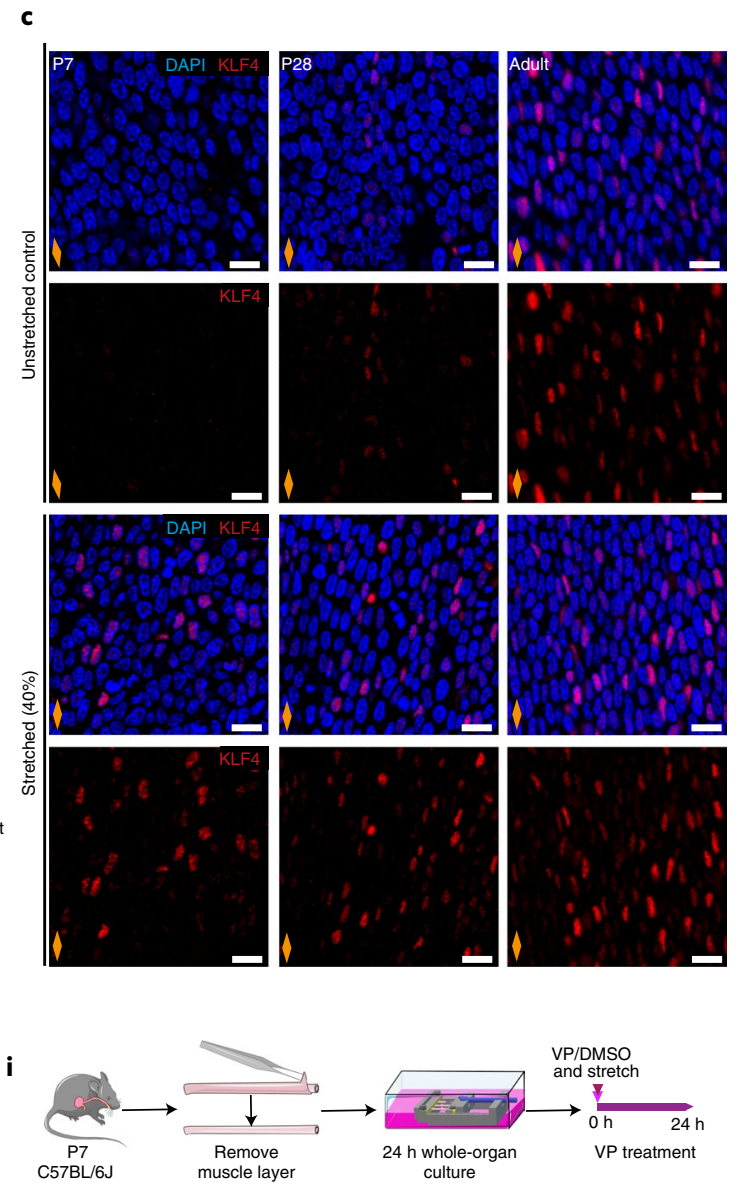

j
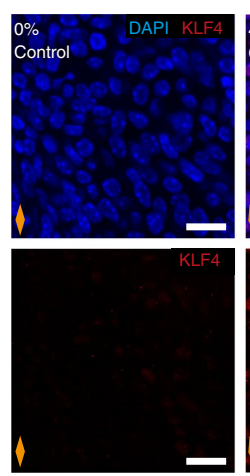
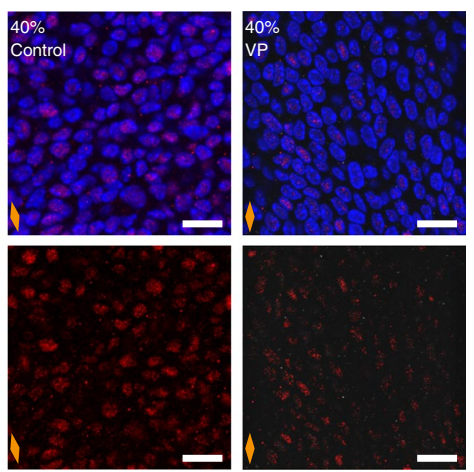

I

Postnatal development

Mechanical transition

Homeostasis

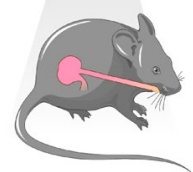

Tissue growth

$\approx$

Animal

growth

Tissue growth

$<$

Animal

growth
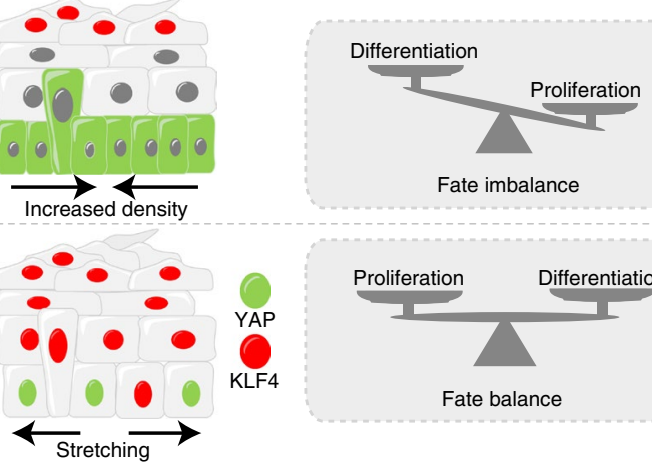
uncover the impacts that diverse types of mechanical forces have on major organs in the body ${ }^{24,29,81,86,87}$, from the shear stress dictating the development of the heart and lungs in mouse and zebrafish embryos ${ }^{18,74,75}$ to the mechanical stress guiding the patterning of the intestines and skin follicles in the developing chick and mouse $\mathrm{e}^{19-21,88}$. However, there is surprisingly limited knowledge on the mechanisms regulating how developmental processes come to an end, safeguarding adult tissue homeostasis from abnormal growth and disease. Our findings in the postnatal mouse oesophagus reveal that this transition is linked to the mechanical changes experienced by the tissue. The rapid expansion of the oesophagus after birth is progressively restricted as the oesophageal epithelium matures and keratinizes. When that occurs, the oesophagus no longer grows at the same rate as the rest of the body, resulting in progressive stretching of the oesophageal epithelium. This is marked by a transcriptional switch, showing an upregulation in the expression of genes known to regulate the response to mechanical stimuli (Klf2, Cav1 and $D c n)^{72,74,75}$, including YAP-associated genes (Cyr61, Thbs1, Igfbp2 and Klf6, among others $)^{66,67,69,71}$. Interestingly, this transcriptional signature is a feature of a particular committed cell population that emerges at P28 in the basal progenitor compartment, and is marked by high levels of KLF4 expression. The presence of a differentiating population in the basal layer promotes a more balanced progenitor cell behaviour, signalling the transition towards adult homeostasis ${ }^{15}$. Ex vivo stretching experiments reveal that the build-up in tissue strain experienced by the oesophagus during its postnatal development is responsible for the emergence of this KLF4+ basal committed population. Remarkably, we demonstrate the existence of a defined strain threshold that triggers the tilt in basal cell fate towards commitment, offering a means by which the temporal regulation of this process may be achieved. Genetic mouse models provide further mechanistic insights, revealing that KLF4 expression is regulated in a YAP-dependent manner. Our results establish a direct link between differential growth, tissue stretching and the acquisition of a committed basal population that defines the transition towards homeostasis. This supports the idea that changes in tissue mechanics can regulate epithelial behaviour in response to specific tissue needs.

Several in vitro and in vivo studies have shown that activation of typical mechanosensors such as YAP/TAZ and Piezol triggers an epithelial proliferative response ${ }^{66,89-94}$. Additional work in the mouse intestinal epithelium indicated that YAP can also regulate cell differentiation, specifically through its interaction with KLF4 (ref. ${ }^{95}$ ). Our observations suggest a model in which changes in cell behaviour are regulated by integrating the physiological levels of mechanical stress happening at the organ level with those sensed by local neighbouring cells in a YAP-dependent fashion. This provides a simple solution to orchestrate the maintenance of a whole tissue at the cellular level (model in Fig. 7l).

Our study offers a new perspective in understanding the mechanisms governing epithelial cell transitions. These observations are likely to have wider implications and open exciting new questions as to whether cell behaviour is regulated similarly in other mechanically active tissues ${ }^{96-100}$. Future studies should elucidate whether regulatory processes controlled through differential growth are a broader phenomenon operating in other tissue models, as well as their translational relevance in regeneration and cancer ${ }^{6,87,101}$.

\section{Online content}

Any methods, additional references, Nature Research reporting summaries, source data, extended data, supplementary information, acknowledgements, peer review information; details of author contributions and competing interests; and statements of data and code availability are available at https://doi.org/10.1038/ s41556-021-00679-w.
Received: 9 April 2020; Accepted: 1 April 2021; Published online: 10 May 2021

\section{References}

1. Varga, J. \& Greten, F. R. Cell plasticity in epithelial homeostasis and tumorigenesis. Nat. Cell Biol. 19, 1133-1141 (2017).

2. Tata, P. R. et al. Dedifferentiation of committed epithelial cells into stem cells in vivo. Nature 503, 218-223 (2013).

3. Van Es, J. H. et al. Dll1 ${ }^{+}$secretory progenitor cells revert to stem cells upon crypt damage. Nat. Cell Biol. 14, 1099-1104 (2012).

4. Ito, M. et al. Stem cells in the hair follicle bulge contribute to wound repair but not to homeostasis of the epidermis. Nat. Med. 11, 1351-1354 (2005).

5. Ge, Y. et al. Stem cell lineage infidelity drives wound repair and cancer. Cell 169, 636-650.e14 (2017).

6. Yui, S. et al. YAP/TAZ-dependent reprogramming of colonic epithelium links ECM remodeling to tissue regeneration. Cell Stem Cell 22, 35-49.e7 (2018).

7. Que, J., Garman, K. S., Souza, R. F. \& Spechler, S. J. Pathogenesis and cells of origin of Barrett's esophagus. Gastroenterology 157, 349-364.e1 (2019).

8. Guiu, J. et al. Tracing the origin of adult intestinal stem cells. Nature 570, 107-111 (2019).

9. Andersen, M. S. et al. Tracing the cellular dynamics of sebaceous gland development in normal and perturbed states. Nat. Cell Biol. 21, 924-932 (2019).

10. Alcolea, M. P. \& Jones, P. H. Tracking cells in their native habitat: lineage tracing in epithelial neoplasia. Nat. Rev. Cancer 13, 161-171 (2013).

11. Blanpain, C. \& Fuchs, E. Stem cell plasticity. Plasticity of epithelial stem cells in tissue regeneration. Science 344, 1242281 (2014).

12. Tata, P. R. et al. Developmental history provides a roadmap for the emergence of tumor plasticity. Dev. Cell 44, 679-693.e5 (2018).

13. Donati, G. et al. Wounding induces dedifferentiation of epidermal Gata6 $^{+}$cells and acquisition of stem cell properties. Nat. Cell Biol. 19, 603-613 (2017).

14. Rosekrans, S. L., Baan, B., Muncan, V. \& van den Brink, G. R. Esophageal development and epithelial homeostasis. Am. J. Physiol. Gastrointest. Liver Physiol. 309, G216-G228 (2015).

15. Doupe, D. P. et al. A single progenitor population switches behavior to maintain and repair esophageal epithelium. Science 337, 1091-1093 (2012).

16. Mammoto, T. et al. Mechanochemical control of mesenchymal condensation and embryonic tooth organ formation. Dev. Cell 21, 758-769 (2011).

17. Barriga, E. H., Franze, K., Charras, G. \& Mayor, R. Tissue stiffening coordinates morphogenesis by triggering collective cell migration in vivo. Nature 554, 523-527 (2018).

18. Li, J. et al. The strength of mechanical forces determines the differentiation of alveolar epithelial cells. Dev. Cell 44, 297-312.e5 (2018).

19. Shyer, A. E. et al. Villification: how the gut gets its villi. Science 342, 212-218 (2013).

20. Shyer, A. E., Huycke, T. R., Lee, C., Mahadevan, L. \& Tabin, C. J. Bending gradients: how the intestinal stem cell gets its home. Cell 161, 569-580 (2015).

21. Shyer, A. E. et al. Emergent cellular self-organization and mechanosensation initiate follicle pattern in the avian skin. Science 357, 811-815 (2017).

22. Heckel, E. et al. Oscillatory flow modulates mechanosensitive $k l f 2 a$ expression through trpv4 and trpp2 during heart valve development. Curr. Biol. 25, 1354-1361 (2015)

23. Vermot, J. et al. Reversing blood flows act through $k l f 2 a$ to ensure normal valvulogenesis in the developing heart. PLoS Biol. 7, e1000246 (2009).

24. Gilmour, D., Rembold, M. \& Leptin, M. From morphogen to morphogenesis and back. Nature 541, 311-320 (2017).

25. Segel, M. et al. Niche stiffness underlies the ageing of central nervous system progenitor cells. Nature 573, 130-134 (2019).

26. Biggs, L. C., Kim, C. S., Miroshnikova, Y. A. \& Wickstrom, S. A.Mechanical forces in the skin: roles in tissue architecture, stability, and function. $J$. Invest. Dermatol. 140, 284-290 (2020).

27. Vining, K. H. \& Mooney, D. J. Mechanical forces direct stem cell behaviour in development and regeneration. Nat. Rev. Mol. Cell Biol. 18, 728-742 (2017).

28. LeGoff, L. \& Lecuit, T. Mechanical forces and growth in animal tissues. Cold Spring Harb. Perspect. Biol. 8, a019232 (2015).

29. Petridou, N. I., Spiro, Z. \& Heisenberg, C. P. Multiscale force sensing in development. Nat. Cell Biol. 19, 581-588 (2017).

30. Hardman, M. J., Sisi, P., Banbury, D. N. \& Byrne, C. Patterned acquisition of skin barrier function during development. Development 125 , 1541-1552 (1998)

31. Liu, K. et al. Sox 2 cooperates with inflammation-mediated Stat 3 activation in the malignant transformation of foregut basal progenitor cells. Cell Stem Cell 12, 304-315 (2013). 
32. DeWard, A. D., Cramer, J. \& Lagasse, E. Cellular heterogeneity in the mouse esophagus implicates the presence of a nonquiescent epithelial stem cell population. Cell Rep. 9, 701-711 (2014).

33. Tetreault, M. P. et al. Esophageal squamous cell dysplasia and delayed differentiation with deletion of Krüppel-like factor 4 in murine esophagus. Gastroenterology 139, 171-181.e9 (2010).

34. Patel, S., Xi, Z. F., Seo, E. Y., McGaughey, D. \& Segre, J. A. Klf4 and corticosteroids activate an overlapping set of transcriptional targets to accelerate in utero epidermal barrier acquisition. Proc. Natl Acad. Sci. USA 103, 18668-18673 (2006)

35. Segre, J. A., Bauer, C. \& Fuchs, E. Klf4 is a transcription factor required for establishing the barrier function of the skin. Nat. Genet. 22, 356-360 (1999).

36. Fortunel, N. O. et al. KLF4 inhibition promotes the expansion of keratinocyte precursors from adult human skin and of embryonic-stemcell-derived keratinocytes. Nat. Biomed. Eng. 3, 985-997 (2019).

37. Kypriotou, M., Huber, M. \& Hohl, D. The human epidermal differentiation complex: cornified envelope precursors, S100 proteins and the 'fused genes' family. Exp. Dermatol. 21, 643-649 (2012).

38. Mesa, K. R. et al. Homeostatic epidermal stem cell self-renewal is driven by local differentiation. Cell Stem Cell 23, 677-686.e4 (2018).

39. Rompolas, P. et al. Spatiotemporal coordination of stem cell commitment during epidermal homeostasis. Science 352, 1471-1474 (2016).

40. Kulukian, A. \& Fuchs, E. Spindle orientation and epidermal morphogenesis. Phil. Trans. R. Soc. Lond. B Biol. Sci. 368, 20130016 (2013).

41. Clayton, E. et al. A single type of progenitor cell maintains normal epidermis. Nature 446, 185-189 (2007).

42. Mascre, G. et al. Distinct contribution of stem and progenitor cells to epidermal maintenance. Nature 489, 257-262 (2012).

43. Alcolea, M. P. et al. Differentiation imbalance in single oesophageal progenitor cells causes clonal immortalization and field change. Nat. Cell Biol. 16, 615-622 (2014).

44. Driskell, R. R. et al. Distinct fibroblast lineages determine dermal architecture in skin development and repair. Nature 504, 277-281 (2013).

45. Crisera, C. A., Maldonado, T. S., Longaker, M. T. \& Gittes, G. K. Defective fibroblast growth factor signaling allows for nonbranching growth of the respiratory-derived fistula tract in esophageal atresia with tracheoesophageal fistula. J. Pediatr. Surg. 35, 1421-1425 (2000).

46. Botchkarev, V. A. \& Sharov, A. A. BMP signaling in the control of skin development and hair follicle growth. Differentiation 72, 512-526 (2004).

47. Park, S. et al. B-cell translocation gene 2 (Btg2) regulates vertebral patterning by modulating bone morphogenetic protein/Smad signaling. Mol. Cell. Biol. 24, 10256-10262 (2004).

48. Li, X. \& Cao, X. BMP signaling and skeletogenesis. Ann. NY Acad. Sci. 1068, 26-40 (2006)

49. Blanco, S. et al. Stem cell function and stress response are controlled by protein synthesis. Nature 534, 335-340 (2016).

50. Koria, P. \& Andreadis, S. T. Epidermal morphogenesis: the transcriptional program of human keratinocytes during stratification. J. Invest. Dermatol. 126, 1834-1841 (2006).

51. Sadagurski, M. et al. Insulin-like growth factor 1 receptor signaling regulates skin development and inhibits skin keratinocyte differentiation. Mol. Cell. Biol. 26, 2675-2687 (2006).

52. Lim, X. et al. Interfollicular epidermal stem cells self-renew via autocrine Wnt signaling. Science 342, 1226-1230 (2013).

53. Zhang, C. et al. Wnt $/ \beta$-catenin signaling is critical for dedifferentiation of aged epidermal cells in vivo and in vitro. Aging Cell 11, 14-23 (2012).

54. Joost, S. et al. Single-cell transcriptomics reveals that differentiation and spatial signatures shape epidermal and hair follicle heterogeneity. Cell Syst. 3, 221-237.e9 (2016).

55. Haake, A. R. \& Cooklis, M. Incomplete differentiation of fetal keratinocytes in the skin equivalent leads to the default pathway of apoptosis. Exp. Cell. Res. 231, 83-95 (1997).

56. Ge, Y. \& Fuchs, E. Stretching the limits: from homeostasis to stem cell plasticity in wound healing and cancer. Nat. Rev. Genet. 19, 311-325 (2018)

57. Philippeos, C. et al. Spatial and single-cell transcriptional profiling identifies functionally distinct human dermal fibroblast subpopulations. J. Invest. Dermatol. 138, 811-825 (2018).

58. Nystrom, A. \& Bruckner-Tuderman, L. Matrix molecules and skin biology. Semin. Cell Dev. Biol. 89, 136-146 (2019).

59. Ghaleb, A. M. \& Yang, V. W. Krüppel-like factor 4 (KLF4): what we currently know. Gene 611, 27-37 (2017).

60. Wei, D., Kanai, M., Huang, S. \& Xie, K. Emerging role of KLF4 in human gastrointestinal cancer. Carcinogenesis 27, 23-31 (2006).

61. Birsoy, K., Chen, Z. \& Friedman, J. Transcriptional regulation of adipogenesis by KLF4. Cell Metab. 7, 339-347 (2008).

62. Morgan, J. T., Raghunathan, V. K., Chang, Y. R., Murphy, C. J. \& Russell, P. Wnt inhibition induces persistent increases in intrinsic stiffness of human trabecular meshwork cells. Exp. Eye Res. 132, 174-178 (2015).
63. Mendez, M. G. \& Janmey, P. A. Transcription factor regulation by mechanical stress. Int. J. Biochem. Cell Biol. 44, 728-732 (2012).

64. Morse, E. M. et al. PAK6 targets to cell-cell adhesions through its $\mathrm{N}$-terminus in a Cdc42-dependent manner to drive epithelial colony escape. J. Cell Sci. 129, 380-393 (2016)

65. Kopf, J., Petersen, A., Duda, G. N. \& Knaus, P. BMP2 and mechanical loading cooperatively regulate immediate early signalling events in the BMP pathway. BMC Biol. 10, 37 (2012).

66. Zhang, H., Pasolli, H. A. \& Fuchs, E. Yes-associated protein (YAP) transcriptional coactivator functions in balancing growth and differentiation in skin. Proc. Natl Acad. Sci. USA 108, 2270-2275 (2011).

67. Maglic, D. et al. YAP-TEAD signaling promotes basal cell carcinoma development via a c-JUN/AP1 axis. EMBO J. 37, e98642 (2018).

68. Schutte, U. et al. Hippo signaling mediates proliferation, invasiveness, and metastatic potential of clear cell renal cell carcinoma. Transl. Oncol. 7, 309-321 (2014).

69. Shen, J. et al. Hippo component YAP promotes focal adhesion and tumour aggressiveness via transcriptionally activating THBS1/FAK signalling in breast cancer. J. Exp. Clin. Cancer Res. 37, 175 (2018).

70. He, C. et al. YAP forms autocrine loops with the ERBB pathway to regulate ovarian cancer initiation and progression. Oncogene 34, 6040-6054 (2015).

71. Xin, M. et al. Regulation of insulin-like growth factor signaling by Yap governs cardiomyocyte proliferation and embryonic heart size. Sci. Signal 4, ra70 (2011).

72. Moreno-Vicente, R. et al. Caveolin-1 modulates mechanotransduction responses to substrate stiffness through actin-dependent control of YAP. Cell Rep. 25, 1622-1635.e6 (2018).

73. Echarri, A. \& Del Pozo, M. A. Caveolae-mechanosensitive membrane invaginations linked to actin filaments. J. Cell Sci. 128, 2747-2758 (2015).

74. Steed, E. et al. klf2a couples mechanotransduction and zebrafish valve morphogenesis through fibronectin synthesis. Nat. Commun. 7, 11646 (2016).

75. Goddard, L. M. et al. Hemodynamic forces sculpt developing heart valves through a KLF2-WNT9B paracrine signaling axis. Dev. Cell 43, 274-289.e5 (2017)

76. Zhao, B. et al. Angiomotin is a novel Hippo pathway component that inhibits YAP oncoprotein. Genes Dev. 25, 51-63 (2011).

77. Das Thakur, M. et al. Ajuba LIM proteins are negative regulators of the Hippo signaling pathway. Curr. Biol. 20, 657-662 (2010).

78. Misra, J. R. \& Irvine, K. D. The Hippo signaling network and its biological functions. Annu. Rev. Genet. 52, 65-87 (2018).

79. Yuan, Y. et al. YAP1/TAZ-TEAD transcriptional networks maintain skin homeostasis by regulating cell proliferation and limiting KLF4 activity. Nat. Commun. 11, 1472 (2020)

80. Verstreken, C. M., Labouesse, C., Agley, C. C. \& Chalut, K. J. Embryonic stem cells become mechanoresponsive upon exit from ground state of pluripotency. Open Biol. 9, 180203 (2019).

81. Aragona, M. et al. Mechanisms of stretch-mediated skin expansion at single-cell resolution. Nature 584, 268-273 (2020).

82. Candi, E., Schmidt, R. \& Melino, G. The cornified envelope: a model of cell death in the skin. Nat. Rev. Mol. Cell Biol. 6, 328-340 (2005).

83. Hatzfeld, M., Keil, R. \& Magin, T. M. Desmosomes and intermediate filaments: their consequences for tissue mechanics. Cold Spring Harb. Perspect. Biol. 9, a029157 (2017).

84. Liu-Chittenden, Y. et al. Genetic and pharmacological disruption of the TEAD-YAP complex suppresses the oncogenic activity of YAP. Genes Dev. 26, 1300-1305 (2012).

85. Wang, L. et al. Unbalanced YAP-SOX9 circuit drives stemness and malignant progression in esophageal squamous cell carcinoma. Oncogene 38, 2042-2055 (2019).

86. Nowlan, N. C., Francis-West, P. \& Nelson, C. Mechanics of development Phil. Trans. R Soc. Lond. B Biol. Sci 373, 20170316 (2018).

87. Fiore, V. F. et al. Mechanics of a multilayer epithelium instruct tumour architecture and function. Nature 585, 433-439 (2020).

88. Huycke, T. R. et al. Genetic and mechanical regulation of intestinal smooth muscle development. Cell 179, 90-105.e21 (2019).

89. Aragona, M. et al. A mechanical checkpoint controls multicellular growth through YAP/TAZ regulation by actin-processing factors. Cell 154, 1047-1059 (2013).

90. Dupont, S. et al. Role of YAP/TAZ in mechanotransduction. Nature 474, 179-183 (2011).

91. Benham-Pyle, B. W., Pruitt, B. L. \& Nelson, W. J. Cell adhesion. Mechanical strain induces E-cadherin-dependent Yap1 and $\beta$-catenin activation to drive cell cycle entry. Science 348, 1024-1027 (2015).

92. Gudipaty, S. A. et al. Mechanical stretch triggers rapid epithelial cell division through Piezol. Nature 543, 118-121 (2017).

93. Elbediwy, A. et al. Integrin signalling regulates YAP and TAZ to control skin homeostasis. Development 143, 1674-1687 (2016).

94. Schlegelmilch, K. et al. Yap1 acts downstream of $\alpha$-catenin to control epidermal proliferation. Cell 144, 782-795 (2011). 
95. Imajo, M., Ebisuya, M. \& Nishida, E. Dual role of YAP and TAZ in renewal of the intestinal epithelium. Nat. Cell Biol. 17, 7-19 (2015).

96. Gokhin, D. S., Ward, S. R., Bremner, S. N. \& Lieber, R. L. Quantitative analysis of neonatal skeletal muscle functional improvement in the mouse. J. Exp. Biol. 211, 837-843 (2008).

97. Domenga, V. et al. Notch3 is required for arterial identity and maturation of vascular smooth muscle cells. Genes Dev. 18, 2730-2735 (2004).

98. Chao, C. M., Moiseenko, A., Zimmer, K. P. \& Bellusci, S. Alveologenesis: key cellular players and fibroblast growth factor 10 signaling. Mol. Cell. Pediatr. 3, 17 (2016).
99. White, R. B., Bierinx, A. S., Gnocchi, V. F. \& Zammit, P. S. Dynamics of muscle fibre growth during postnatal mouse development. BMC Dev. Biol. 10, 21 (2010).

100. Dekoninck, S. et al. Defining the design principles of skin epidermis postnatal growth. Cell 181, 604-620.e22 (2020).

101. Paszek, M. J. et al. The cancer glycocalyx mechanically primes integrin-mediated growth and survival. Nature 511, 319-325 (2014).

Publisher's note Springer Nature remains neutral with regard to jurisdictional claims in published maps and institutional affiliations.

๑) The Author(s), under exclusive licence to Springer Nature Limited 2021 


\section{Methods}

Mouse strains and allele induction. All animal experiments were approved by the local ethical review committees. Work was approved by the University of Cambridge and conducted according to Home Office project licence PPL70/8866 at the Wellcome-Medical Research Council (MRC) Cambridge Stem Cell Institute, Cambridge University. Additional mouse work was conducted according to approved protocols LCMB-034 and 19-329 from the National Institutes of Health Intramural Animal Care and Use Committee of the National Cancer Institute, or under permit number 2017-15-0201-01381, reviewed and approved by the National Animal Ethics Committee in Denmark.

Unless otherwise specified, wild-type C57BL/6J mice (Charles River; strain code 632) were used. We also used the following genetically engineered mouse lines: Fucci2a (R26Fucci2aR; kindly provided by I. J. Jackson ${ }^{102}$ ); tetO-YAP ${ }^{\text {S127A }}$ (kindly provided by J. Larsson ${ }^{103}$ ); Rosa26-rtTAM2 (stock number 006965; The Jackson Laboratory); Krt5-rtTA;TEADi (kindly provided by R. Iglesias-Bartolome ${ }^{79}$ ); and Rosa26-mT/mG (stock number 007676; The Jackson Laboratory $\left.{ }^{104}\right)$. Animals cohorts were used at the exact postnatal days indicated, with the adult cohort constituting mice between 10 and 14 weeks of age.

All strains were maintained in a C57BL/6 background. All experiments comprised male and female mice, with no gender-specific differences observed. Only male mice were used in the scRNA-seq experiments, to avoid confounding effects due to oestrous cycles. All animals were housed between 19 and $23^{\circ} \mathrm{C}, 45$ and $65 \%$ humidity and a $12 \mathrm{~h}$ day/ $12 \mathrm{~h}$ night light cycle.

Tissue dimensions. Oesophageal dimensions were measured from P2 to adult. Wild-type mice were culled and the oesophagi were excised, taking as reference two anatomical landmarks: the cricoid cartilage and the opening of the stomach fundus. Oesophageal tubes were longitudinally opened and flattened, to measure the length and width under a dissecting microscope, and the tissue area was calculated. The average tissue area was calculated for $n=3-22$ per time point

For in situ versus ex vivo comparison, we performed an immediate in situ postmortem tissue measurement, followed by a subsequent measurement after dissection (ex vivo) (Fig. 5a). Wild-type mice were culled at the indicated time points and organs surrounding the oesophagus and stomach were removed for a clear view. In situ images were taken, from which the length (oesophagus) and perimeter outline (stomach) were measured. This was repeated immediately after tissue dissection. Measurements were expressed as a percentage relative to their value at P7.

Histology. Haematoxylin and eosin staining was performed by the Histology Core Service at the Cambridge Stem Cell Institute and imaged using an EVOS XL Core Imaging System.

Tissue preparation and immunofluorescence. For in situ fixation of the oesophagus (Extended Data Fig. 5a,b), wild-type mice were culled and the organs surrounding the oesophagus and stomach were removed. The thoracic cavity was filled with $4 \%$ paraformaldehyde (Alfa Aesar; 043368) in phosphate-buffered saline (PBS) for $10 \mathrm{~min}$ for fixation. The oesophagus was then excised and processed for immunostaining, as indicated below.

For oesophageal wholemounting, oesophagi were excised and opened longitudinally. Then, the muscle layer was removed and the tissue was flattened under a dissecting microscope. The epithelial-stromal composite was then fixed in $4 \%$ paraformaldehyde in PBS for $30 \mathrm{~min}$. Samples were incubated for $30 \mathrm{~min}$ in permeabilization buffer ( $0.5 \%$ bovine serum albumin (VWR International; $126575-10), 0.25 \%$ fish skin gelatin (Sigma-Aldrich; G7765) and 1\% Triton X-100 (Fisher Scientific; 10102913) in PBS) and blocked for $2 \mathrm{~h}$ in permeabilization buffer containing 10\% donkey serum (PBDS; Scientific Laboratory Supplies; D9663). Primary antibodies (Supplementary Table 5) were incubated in PBDS for $3 \mathrm{~d}$ at $4^{\circ} \mathrm{C}$, followed by four washes over $24 \mathrm{~h}$ with $0.2 \%$ Tween-20 (Promega; H5151) in PBS. Secondary antibodies were incubated overnight at $4{ }^{\circ} \mathrm{C}$ in PBDS and washed four times over $4 \mathrm{~h}$ with $0.2 \%$ Tween-20 in PBS. Cell nuclei were stained with $1 \mu \mathrm{g} \mathrm{ml}^{-1} 4^{\prime}$,6-diamidino-2-phenylindole (DAPI; Sigma-Aldrich; D9542) in PBS at $4^{\circ} \mathrm{C}$ before mounting. Wholemounts were cleared in 1.52 RapiClear mounting media (SunJin Lab; RC152001). Organ culture samples were stained following the same procedure.

Wholemounts of the oesophageal epithelium were stained with specific antibodies. These were prepared by incubating oesophagi in $5 \mathrm{mM}$ ethylenediaminetetraacetic acid (EDTA; Life Technologies; 15575020) in PBS for $2.5 \mathrm{~h}$ at $37^{\circ} \mathrm{C}$ following muscle layer removal. The epithelium was peeled from the stroma and fixed as above. Staining was performed using low Triton permeabilization buffer ( $0.5 \%$ bovine serum albumin, $0.25 \%$ fish skin gelatine and $0.5 \%$ Triton X-100 in PBS) and the following altered timings: $10 \mathrm{~min}$ of permeabilization in low Triton permeabilization buffer; $1 \mathrm{~h}$ of blocking in PBDS; primary antibodies in PBDS overnight at $4{ }^{\circ} \mathrm{C} ; 2 \mathrm{~h}$ of washes using $0.2 \%$ Tween-20 in PBS; $3 \mathrm{~h}$ of secondary antibodies in PBDS; washes as for the primary antibodies; and cell nuclei staining as above.

For the staining of $10 \mu \mathrm{m}$ cryosections, fixed oesophagi were embedded in optimal cutting temperature compound (Thermo Scientific; 12678646) for cryosectioning onto glass slides. Sections were permeabilized in Triton permeabilization buffer for $10 \mathrm{~min}$, blocked in PBDS for $30 \mathrm{~min}$ and incubated overnight with primary antibodies in Triton permeabilization buffer at $4{ }^{\circ} \mathrm{C}$, followed by a 1-h incubation with secondary antibodies in Triton permeabilization buffer. Sections were washed four times for $5 \mathrm{~min}$ in $0.2 \%$ Tween-20 in PBS between incubations.

Where indicated, EdU incorporation was detected using Click-iT imaging kits according to the manufacturer's instructions (Invitrogen).

EdU tracing. For in vivo tracing, EdU (Life Technologies; A10044) was administered at $10 \mu \mathrm{g} \mathrm{g}^{-1}$ bodyweight and $0.1 \mathrm{mg} \mathrm{ml}^{-1}$ via subcutaneous injection. Tissues were collected 2 and $24 \mathrm{~h}$ after injection. In organ cultures, EdU was added to the culture media at a final concentration of $10 \mu \mathrm{M}$ and incubated for $2 \mathrm{~h}$ at $37^{\circ} \mathrm{C}$ and $5 \% \mathrm{CO}_{2}$. EdU ${ }^{+}$cells were quantified in wholemounts from three to nine confocal z-stacks per animal.

For tracking $\mathrm{EdU}^{+}$post-mitotic cell pairs in adult mice, EdU was administered at 18:00 (subcutaneously), when the proportion of S-phase cells was low in the circadian cycle, making clonal density labelling feasible, and chased for $12 \mathrm{~h}^{43,105}$. The KLF4 expression profile was determined in $225 \mathrm{EdU}$ pairs from three different animals.

Basal cell density. The basal cell density in oesophageal wholemounts was calculated by quantifying the number of $\mathrm{DAPI}^{+}$basal cells per field in three to six images per animal.

Total cell production and imbalance. First, we estimated the total number of basal cells in the whole oesophagus over time, by multiplying the basal cell density by the total tissue area at each time point. The total basal cell production rate, $\dot{B}$, was then estimated as the average change in basal cell number, $B$, divided by the time step.

The degree of fate imbalance (that is, the degree to which basal cell fate is biased towards duplication over differentiation and cell loss) was estimated as $(1 / \lambda) \dot{B} / B$, where $\lambda$ is the cell division rate. The division rate was determined from the results of a short-term ( $2 \mathrm{~h}$ ) EdU incorporation assay (Fig. 2e) and normalized by the reported division rate of basal cells in the homeostatic adult tissue ${ }^{15}$.

Tissue thickness. Tissue thickness was measured using side views of rendered confocal oesophageal wholemount images. Eighteen individual measurements were made for each of three animals.

Imaging, analysis and segmentation. Confocal images were acquired using either an inverted Leica SP5 with standard laser configuration, an SP8 X with a white light laser or an upright SP8 X with a standard laser configuration with LAS X 3.5.5.19976 Leica software. Typical confocal settings used included: a 40× objective, an optimal pinhole, a scan speed of $400 \mathrm{~Hz}$, a line average of three, optimal step size and a resolution of $512 \times 512$ or $1,024 \times 1,024$ pixels. For quantification, images where acquired with a digital zoom of $2 \times$ or $3 \times$.

Images were reconstructed from optical sections using Volocity 5.3 .3 software (PerkinElmer) and ImageJ 1.51w. KLF4, EdU, Fucci2a (mCherry and mVenus) and YAP intensity measurements (Fig. 6b,c) were performed on Volocity 5.3.3 using semi-automated nuclear segmentation. For the analysis of KLF4 bright cells $\left(\mathrm{KLF}^{+}\right)$, the threshold was set to include nuclei above the 80th percentile of KLF4 intensity (Fig. 2b,f,g and Extended Data Fig. 2c-e). This was visually confirmed to be over the background level of expression for nuclear KLF4 staining. EdU was set as $>0.2$, as detected in representative confocal images. F-actin (phalloidin labelling) and pMLC2 were quantified using the image segmentation protocol described in Supplementary Note 1 .

YAP localization was assessed by measuring the fluorescent intensity of YAP immunolabelling and DAPI staining across the major cell axis of each individual cell. Nuclear and cytoplasmic YAP intensities were assigned per pixel based on the DAPI intensity. Those within the lower 20th percentile of DAPI intensity were considered cytoplasmic, while those within the top 20th percentile of DAPI intensity were measured as nuclear (Fig. $5 \mathrm{~m}$ and Extended Data Fig. 6j-1). A total of 240 cells were analysed from three separate animals at four different time points (P7, P14, P28 and adult; 60 cells per time point).

Collagen fibres were imaged using SHG, alongside NucRed647 (Life Technologies; R37106) in wholemounts. Imaging was performed using an inverted Leica TCS SP5 acousto-optic beam splitter multiphoton laser scanning microscope equipped with a mode-locked Ti:Sapphire laser (Chameleon Ultra II; Coherent; $680-1,080 \mathrm{~nm}$ tuning range; $80 \mathrm{MHz}$ repetition rate; $140 \mathrm{fs}$ pulse width) that allows for SHG imaging. For image acquisition, the excitation laser (Ti:Sapphire) was tuned to $900 \mathrm{~nm}$, and was acquired at an emission of $450 \mathrm{~nm}$ for SHG signal. Typical settings included: a $40 \times$ objective, a $600-\mu \mathrm{m}$ pinhole, a $400-\mathrm{Hz}$ scan speed, a line average of two, a $0.15-\mu \mathrm{m}$ step size and a resolution of $1,024 \times 1,024$ pixels. Three images per animal were captured and the collagen fibre orientation was assessed using the pipeline outlined in Supplementary Note 1.

For single-cell image segmentation of wholemounts, single 2 - $\mu \mathrm{m} \mathrm{z}$-slices of the basal layer, acquired using a Leica SP5 confocal microscope system, were used. For single-cell image segmentation of cryosections, single 2 - $\mu \mathrm{m}$ z-slices of tissue cross-sections were acquired with the criteria outlined above. A minimum of three images were captured per animal. Analysis was performed via the pipeline described in Supplementary Note 1. 
Note that images from in situ oesophagi (fixed postmortem undissected) were compared with oesophagi fixed immediately after dissection to confirm that the basal cell shape was not affected by tissue harvesting (Fig. 4a versus Extended Data Fig. 5a,b).

RNA. Single-cell and RNA isolation. Epithelial-stromal composites were obtained as indicated above. For each sample, oesophagi were pooled from different animals (C57BL/6 wild-type mice) to enable the capture of sufficient cell numbers (five mice for P7, four mice for P28 and three mice for adult; three independent samples for P7 and P28; six independent samples for adult).

Following initial preparation, wholemounts were cut into $3 \mathrm{~mm}^{2}$ pieces and incubated in $0.5 \mathrm{mg} \mathrm{ml}^{-1}$ dispase (Sigma-Aldrich; D4818) in PBS containing $1 \mathrm{U} \mathrm{ul}^{-1}$ RNAse Inhibitor (Life Technologies; AM2696) at $37^{\circ} \mathrm{C}$ for $10 \mathrm{~min}$. The epithelium was then peeled away from the stroma and thoroughly minced before placing back into dispase for a 5 -min incubation at $37^{\circ} \mathrm{C}$. EDTA was then added to a final concentration of $5 \mathrm{mM}$ and the suspension was diluted $1 / 5$ by adding fluorescence-activated cell sorting (FACS) staining buffer ( $2 \%$ heat-inactivated foetal bovine serum (Life Technologies; 26140079), 25 mM HEPES (Life

Technologies; 15630056) and $1 \mathrm{mM}$ EDTA in PBS) in order to reduce the dispase activity. Samples were then filtered through a $30-\mu \mathrm{m}$ cell strainer and centrifuged at $300 \mathrm{~g}$ for $10 \mathrm{~min}$ at $4{ }^{\circ} \mathrm{C}$, then resuspended in staining buffer. Staining with primary antibodies or isotype controls was performed for $15 \mathrm{~min}$ at $4^{\circ} \mathrm{C}$, followed by a final wash in FACS buffer (FACS staining buffer without EDTA) at $300 \mathrm{~g}$ for $5 \mathrm{~min}$ at $4^{\circ} \mathrm{C}$. The final cell suspension was placed in FACS buffer containing $1 \mathrm{U} \mathrm{ul}^{-1}$ RNAse inhibitor.

The following immunoglobulins were used (Supplementary Table 5) for sorting: $20 \mu \mathrm{g} \mathrm{ml}{ }^{-1}$ EpCam-AF647 to stain epithelial cells; and $8 \mu \mathrm{g} \mathrm{ml}^{-1} \mathrm{CD} 45$-APC-Cy7 to exclude immune contamination. Isotype controls were used at same concentration as specific antibodies: AF647 rat IgG2a and APC-Cy7 mouse IgG2a.

Work was carried out using RNAse-free and/or PCR-grade sterile reagents and plasticware wherever possible.

Viable epithelial $\left(\mathrm{EpCam}^{+} / \mathrm{CD}^{-} 5^{-} / \mathrm{DAPI}^{-}\right)$cells were sorted on a BD FACSAria III cell sorter with BS FACSDiva 8.0.1 software, utilizing DAPI incorporation $\left(4 \mu \mathrm{g} \mathrm{ml}^{-1}\right)$ to exclude dead cells. Approximately 5,000 cells per sample followed the 10x Chromium pipeline for scRNA-seq. FlowJo version 10.6.2 software was used to generate the sorting plots (Extended Data Fig. 3a).

Library preparation and sequencing of RNA from single cells. Single-cell RNA-seq libraries were generated and sequenced using 10x Genomics kits (Single Cell 3' v3) at the Cancer Research UK (CRUK) Cambridge Institute Genomics Core Facility. Libraries were generated in two different batches on different dates. Batch 1 consisted of three biological replicates for P7 and three for adult, used for six libraries. Batch 2 consisted of three biological replicates for P28 and three for adult, used for six libraries. The presence of adult samples in both batches provided common samples to control for any batch effect. The cells for each biological replicate were loaded into one 10x Chromium microfluidics chip channel to package them into one library. In total, 12 libraries were sequenced on Illumina NovaSeq 2 SP and 2 S2 flow cells (Supplementary Table 1).

Single-cell RNA-seq analysis. Data processing. The raw sequencing data from the 10x Genomics platform were processed using CellRanger (version 3.0.2) (Supplementary Table 1). CellRanger aligned reads, filtered empty dropouts and counted unique molecular identifiers (UMIs) to generate a count matrix. We used Ensembl GRCm $38 / \mathrm{mm} 10$ (release 92) as the reference genome for read alignment. To filter out low-quality cells, basic quality control metrics were calculated using the R package scater $(1.12 .2)^{106}$, and cells with $<1,251$ genes were removed. Cells with mitochondrial proportions of $>15 \%$ and genes expressed in fewer than three cells were also discarded from analysis. Read counts were normalized via a deconvolution method using the R package scran (version 1.12.1) ${ }^{107}$. Cells expressing fibroblast marker genes, including Colla2, Col1a1, Fn1 and Pdgfra, were considered to be non-epithelial contaminants. To filter out non-epithelial cells, the normalized read count values for the genes above were averaged and the cells with averaged normalized values for the four genes above the 99th percentile $(>0.4301828)$ of the distribution were filtered out. As a result, a total of 26,482 cells from three to six samples per time point were selected for further analysis. For basic statistics and quality control metrics, see Supplementary Table 1.

Dimension reduction and data visualization. Principal components analysis, combined with technical noise modelling, was applied to the normalized data for dimension reduction, implemented by the denoise_PCA function in the R package scran. The data were then projected using two-dimensional UMAP or t-distributed stochastic neighbour embedding, with default parameter settings, using the $\mathrm{R}$ package scater. The biological replicates for each time point overlapped well with each other, confirming negligible batch effects between samples and conditions; therefore, no batch effect correction was necessary. The R package Seurat (version 3.0.2) ${ }^{108}$ was used to visualize cells in the dimension reduction space.

Data clustering and cluster annotation. Next, a Louvain community detection method was used for clustering ${ }^{109}$. First, a shared nearest-neighbour graph was constructed using $k=20$ nearest neighbours of each cell (the buildSNNGraph function in the R package scran). In this graph, two cells were connected by an edge if they shared nearest neighbours, with the edge weight determined by the highest average rank of the shared neighbours. Then, the Walktrap method from the R package igraph (version 1.2.4.1) (with steps $=4$ as the default option) was used to identify densely connected communities that were considered to be cell clusters.

Cell clusters were annotated based on differentially expressed genes (DEGs) and known marker genes for cell types. If a few neighbouring clusters in the dimension reduction spaces shared key expression patterns, they were merged into one cell type manually. First, based on basal cell markers such as Krt14, Itgb1 and Itga 6 and differentiation markers such as Tgm3, Krt13 and $\mathrm{Grhl3}$, all clusters were classified into basal cells (clusters 1-3, 5-7, 10-13 and 15-17) and differentiated cells (clusters 4, 8, 9 and 14) (Extended Data Fig. 3b,d-f). The predominant expression of basal progenitor markers throughout the entire dataset (including Krt14, Itgb1 and Itga6) reflects an enrichment of basal cells under the experimental conditions used (Extended Data Fig. 3d,e). Accordingly, increased expression of differentiation markers (such as Krt13, Krt4, Tgm3, Grhl3 and Sbsn) was restricted to a few clusters (Extended Data Fig. 3d,e).

Then, we classified all cells into different cell cycle phases based on cell cycle genes: clusters $1,2,4,6,7,8,9,12$ and 14 for G0/G1; clusters 11 and 13 for G1/S; and clusters 3, 5, 10, 15, 16 and 17 for S/G2/M (Extended Data Fig. 3h,i). As a result, all cells were annotated as one of three cell types as follows: clusters $3,5,10$, $11,13,15,16$ and 17 for basal cycling cells; clusters 1, 2, 6, 7 and 12 for resting basal cells; and clusters 4, 8, 9 and 14 for differentiated cells (Extended Data Fig. 3d-i). Of note, although suprabasal cells at early time points do not proliferate (Extended Data Fig. 3j,k), they tend to express key markers of basal identity (Fig. 1i and Extended Data Fig. $3 \mathrm{~d}-\mathrm{f}, \mathrm{i})$. For this reason, clusters containing resting basal cells at P7 probably represent a combination of both basal and basal-like suprabasal cells.

To confirm that the annotated three cell types matched well between different time points, we integrated the data for P7, P28 and adult using a standard workflow of Seurat (version 3.0.2) (Extended Data Fig. 3c). Specifically, we normalized the data for each time point separately using SCTransform and then integrated them by applying the following built-in functions sequentially with default parameters: SelectIntegrationFeatures (nfeatures $=3000$ ); PrepSCTIntegration; FindIntegrationAnchors; and IntegrateData. For cluster annotation, we visualized gene expression in the dimension reduction and in violin plots using built-in functions of Seurat (version 3.0.2).

Classifying gene expression for basal cells into distinct dynamic patterns along the time course and Gene Ontology analysis of the different expression patterns. After annotating cell types, we focused on molecular change for basal cells over time. To this end, we first identified DEGs for each time point using the function findMarkers of scran (version 1.12.1) by defining them as the genes having a false discovery rate (FDR) of $<0.05$ and an absolute value of $\log _{2}$ [fold change] $>$ (95th percentile of all $\log _{2}$ [fold change]) in the three comparisons of P7 versus P28, P28 versus adult and P7 versus adult. The total number of DEGs was 1,738 . We then scaled the normalized expression value of DEGs for all cells and calculated the average of the scaled expression value across the cells for each time point. Then, the scaled $\log _{2}$ [fold change] for each time was calculated by subtracting the averaged scaled value at $\mathrm{P} 7$ from those at all time points (thus, the scaled $\log _{2}$ [fold change] at $\mathrm{P} 7=0$ ).

The scaled $\log _{2}$ [fold change] profiles for DEGs (1,738 genes) were clustered based on $k$-means clustering $(k=10)$ using pheatmap (1.0.12), and the resulting clusters were grouped into four major patterns: pattern 1 (148 genes); pattern 2 (545 genes); pattern 3 (153 genes); and pattern 4 ( 892 genes) (Extended Data Fig. 4a,b and Supplementary Table 2). The genes in each major pattern were used for Gene Ontology analysis by DAVID (https://david.ncifcrf.gov/) ${ }^{110}$. The resulting Gene Ontology terms were manually curated and selected based on experimental observations and biological knowledge (Extended Data Fig. $4 \mathrm{~b}$ and Supplementary Table 3 ).

We then examined the transcriptional heterogeneity in resting basal cells over time. First, we calculated the proportion of resting basal cell clusters at each time point (Fig. 3d,e): clusters 2 and 6 at P7; and clusters 1, 7 and 12 at P28 and adult. This was marked by the emergence of cluster 12 over time, which represents a subset of resting basal cells expressing higher levels of Klf4. To understand temporal changes in resting basal cells from P7 through P28 to the adult stage at the molecular level, we then characterized gene expression changes from cluster 2 over cluster 7 to cluster 12 . To this end, we calculated marker genes for the resting basal cell clusters (2,7 and 12) using the function FindMarkers, as described above: marker genes for each cluster were defined as the genes having FDR $<0.05$ and $\log _{2}$ [fold change] $>$ (97.5 percentile of all $\log _{2}$ [ fold change] $)$ in the comparison of each cluster with the rest of the resting basal cell clusters. The total number of all marker genes was 1,729. Next, based on our observation in Fig. $3 \mathrm{c}-\mathrm{e}$, we grouped clusters of resting basal cells into three groups to represent each time point as follows: clusters 2 and 6 to represent P7; clusters 1 and 7 for P28/ adult; and cluster 12 for P28/adult. For the 1,729 marker genes, their normalized expression value was scaled among resting basal cells and then averaged across the cells of each group. The average expression for the three groups were clustered 
based on $e$-means clustering $(k=6)$ using pheatmap (1.0.12) and the resulting six clusters were grouped into three major patterns (Fig. 3f): pattern 1 (748 genes); pattern 2 (163 genes); and pattern 3 ( 818 genes). Then, we performed Gene Ontology analysis using DAVID for each of the three major patterns to understand underlying biological processes (Supplementary Table 4). As a result, we found that enriched biological processes are very similar between basal cells (Extended Data Fig. 4b) and resting basal cells only (Supplementary Table 4). Of note, well-known Klf4 and Yap-related genes, among other genes associated with a response to mechanical stimuli, belonged to the three major patterns from the resting basal cell clusters, showing dynamic expression across the clusters (Fig. 3f).

We then explored a potential regulatory relationship between genes denoting a response to mechanical stimuli, including YAP targets) and Klf4-related genes in resting basal cells. We examined the temporal order of expression of YAP and Klf4 target genes at the single-cell level from the resting basal cell state and along the differentiation trajectory. Thus, we performed pseudotime analysis using the $\mathrm{R}$ package monocle (version 2.12.0) for resting basal cells and differentiated cells for P7 and P28/adult separately to project the expression profile of relevant genes along differentiation. To this end, we first identified DEGs for resting basal cells and differentiated cells at P7 and P28/adult separately by applying the criteria of FDR $<0.05$ and an absolute value of $\log _{2}$ [fold change] $>$ (95th percentile of all absolute value of $\log _{2}$ [fold change]). As a result, the number of DEGs was 1,018 for P7 and P28/adult, respectively. Based on the DEGs, dimension reduction was performed using the DDRTree algorithm implemented in the R package monocle. Next, the pseudotime order was calculated using the orderCells function of monocle with default parameters. We then displayed the expression profiles for relevant mechanics and Klf4-related genes along the pseudotime for P7 and P28/ adult, separately (Fig. $3 \mathrm{~h}$ and Extended Data Fig. 4f); for each gene, the auto-scaled gene expression was plotted using a rolling mean along its trajectory with a window size of $5 \%$ of the cells.

Whole oesophageal organ culture and tissue stretching. A 3D-printed stretcher was developed from the prototype in ref. ${ }^{80}$ using Autodesk Fusion 360 and Ultimaker Cura software and printed using an Ultimaker 3 3D printer in acrylonitrile butadiene styrene (biocompatible plastic). A millimetric scale was included in the printing of the device to guide the stretch. Once printed and sterilized, a $10-\mathrm{cm}$ adjustable screw was inserted into the lower clamp to allow for controlled tissue stretching (Fig. 7b). The system was then placed in a sterile plastic container with a lid, allowing for the adjustable screw to be accessible from the outside of the container.

Oesophageal epithelial samples were collected at the indicated time points and the muscle layer was removed while retaining the tubular structure. Samples were then placed into the upper and lower clamps under no tension. Control samples were clamped at one end (top clamp in Fig. 7b, inset) and cultured under no longitudinal tension. Stretched samples were tightened into clamps using M3 nuts and screws. The millimetric scale and adjustable screw were used to apply a $40 \%$ stretch in tissue length. Whole-organ cultures were kept for 24 or $48 \mathrm{~h}$ at $37^{\circ} \mathrm{C}$ under $5 \% \mathrm{CO}_{2}$ in minimal medium containing one part Dulbecco's modified Eagle's medium ( $4.5 \mathrm{gl}^{-1} \mathrm{D}$-glucose, pyruvate and L-glutamine; Invitrogen; 11971 025 ) and one part Dulbecco's modified Eagle's medium/F-12, $5 \mu \mathrm{g} \mathrm{ml}^{-1}$ insulin, $1.8 \times 10^{-4} \mathrm{M}$ adenine, $5 \%$ foetal calf serum, $5 \%$ penicillin-streptomycin and $5 \mu \mathrm{g} \mathrm{ml} \mathrm{m}^{-1}$ apo-transferrin.

In vitro stretched tissues were treated with pharmacological compounds to inhibit myosin II motor activity $(25 \mu \mathrm{M}$ BLEBB for $48 \mathrm{~h})$ or YAP activity $(1 \mu \mathrm{M}$ verteporfin for $24 \mathrm{~h}$ ), respectively. Vehicle (dimethyl sulfoxide (DMSO)) was added to control samples.

The tissue response to different levels of tensile strain was assessed in P7 samples subjected to increasing levels of longitudinal stretching (20,40 and $100 \%$ ) for $24 \mathrm{~h}$. For EdU incorporation assays, EdU was added to media at a final concentration of $10 \mu \mathrm{M}$ for $1 \mathrm{~h}, 24 \mathrm{~h}$ before collection.

BLEBB treatment of adult oesophageal organ culture. Epithelial-stromal composites were cut into pieces of $\sim 3 \mathrm{~mm} \times 4 \mathrm{~mm}$ and placed onto transparent ThinCert inserts (Greiner Bio-One; 657641) with the stromal side to the membrane. Tissues were dried for $5 \mathrm{~min}$ at $37^{\circ} \mathrm{C}$ and cultured in minimal medium at $37^{\circ} \mathrm{C}$ under $5 \% \mathrm{CO}_{2}$ for $3 \mathrm{~h}$ to ensure adequate tissue adhesion to the membrane Then, $25 \mu \mathrm{M}$ BLEBB was added to the media. Control explants were treated with DMSO vehicle alone. Samples were collected after $45 \mathrm{~h}$.

For EdU incorporation assays, EdU was added and detected as above.

Changes in basal cell area upon tissue composite separation. Oesophagi from Rosa26-mT/mG mice were dissected and the uppermost keratinized layers of the epithelium were mechanically separated with fine forceps. Alternatively, stromal layers were removed by incubating oesophagi in $5 \mathrm{mM}$ EDTA in PBS for $2.5 \mathrm{~h}$ at $37^{\circ} \mathrm{C}$.

Basal cells were immediately imaged by confocal microscopy. Endogenous expression of the tdTomato reporter in cell membranes allowed for imaging without fixation and immunolabeling. The cell area was measured using the manual region of interest segmentation feature of the Volocity software and compared with that of basal cells from control unprocessed tissue samples.
Statistics and reproducibility. A minimum of three independent mice or tissue cultures were used in all cases, with the exception of the TEADi experiments (see Fig. 6d-f) due to limitations associated with the COVID-19 pandemic. The numbers of biological replicates and animals are indicated in figure captions ( $n$ refers to the number of independent replicates per time point and/or condition). All replicates showed consistent results.

For image analysis, a minimum of three independent samples were inspected in all cases, capturing at least three replicate images each. All figures show representative images of a minimum of three mice or tissue cultures. The data are expressed as median values \pm s.e.m. unless otherwise indicated.

Differences between groups were assessed by two-tailed unpaired $t$-test, oneor two-way analysis of variance (ANOVA) or Chi-squared analysis, as indicated in the figure captions. ANOVA-based analysis was followed by Tukey's test for multiple comparisons. The correlation between YAP and KLF4 expression levels in immunostained wholemounts was calculated by computing the Pearson correlation coefficient $(r)$. All tests were two sided. Exact $P$ values are indicated in the relevant figures up to four decimal places. Statistical differences between groups were assessed utilizing GraphPad Prism software. No statistical method was used to predetermine sample size. The experiments were performed without randomization or blinding.

Reporting Summary. Further information on research design is available in the Nature Research Reporting Summary linked to this article.

\section{Data availability}

The scRNA-seq data that support the findings of this study have been deposited in the ArrayExpress repository under accession code E-MTAB-8662. Source data are provided with this paper. All other data supporting the findings of this study are available from the corresponding author upon reasonable request.

\section{Code availability}

Statistical analysis of the scRNA-seq data was performed using R codes developed for this study. Image segmentation and computational image analysis were performed using Python codes developed and/or adapted for this study. All codes for the computational analysis are available at https://github.com/BenSimonsLab/ McGinn_Nat-Cell-Biol_2021.

\section{References}

102. Mort, R. L. et al. Fucci2a: a bicistronic cell cycle reporter that allows Cre mediated tissue specific expression in mice. Cell Cycle 13, 2681-2696 (2014).

103. Jansson, L. \& Larsson, J. Normal hematopoietic stem cell function in mice with enforced expression of the Hippo signaling effector YAP1. PLoS ONE 7, e32013 (2012)

104. Muzumdar, M. D., Tasic, B., Miyamichi, K., Li, L. \& Luo, L. A global double-fluorescent Cre reporter mouse. Genesis 45, 593-605 (2007).

105. Burns, E. R., Scheving, L. E., Fawcett, D. F., Gibbs, W. M. \& Galatzan, R. E. Circadian influence on the frequency of labeled mitoses method in the stratified squamous epithelium of the mouse esophagus and tongue. Anat. Rec. 184, 265-273 (1976).

106. McCarthy, D. J., Campbell, K. R., Lun, A. T. \& Wills, Q. F. Scater: pre-processing, quality control, normalization and visualization of single-cell RNA-seq data in R. Bioinformatics 33, 1179-1186 (2017).

107. Lun, A. T., Bach, K. \& Marioni, J. C. Pooling across cells to normalize single-cell RNA sequencing data with many zero counts. Genome Biol. 17, 75 (2016).

108. Stuart, T. et al. Comprehensive integration of single-cell data. Cell 177, 1888-1902.e21 (2019)

109. Subelj, L. \& Bajec, M. Unfolding communities in large complex networks: combining defensive and offensive label propagation for core extraction. Phys. Rev. E Stat. Nonlin. Soft Matter Phys. 83, 036103 (2011).

110. Huang da, W., Sherman, B. T. \& Lempicki, R. A. Systematic and integrative analysis of large gene lists using DAVID bioinformatics resources. Nat. Protoc. 4, 44-57 (2009).

\section{Acknowledgements}

We thank members of the Alcolea and Simons laboratories and B. Colom (Sanger Institute) for comments and suggestions; the staff of the University Biomedical Services, Gurdon Institute; P. Humphreys at the imaging core facilities at the Jeffrey Cheah Biomedical Centre; A. Sossick, R. Butler and N. Lawrence at the Gurdon Institute Imaging Facility; the CRUK Cambridge Institute Genomics Core Facility; J. Larsson (tetO-YAP ${ }^{\mathrm{S127A}}$ ); I. J. Jackson (Fucci2a); the NIHR Cambridge BRC Cell Phenotyping Hub for FACS support; and the Jeffrey Cheah Biomedical Centre core facilities. We thank T. Savin, E. Hannezo, B. Ladoux, G. Charras, S. Hénon, N. Harmand, G. Duclos and J.-B. Lugagne for insight on deep-learning-based image analysis and/or tissue mechanics. This work was mainly supported by funding from the Wellcome Trust and Royal Society (105942/Z/14/Z to M.P.A.) and a core support grant from the Wellcome 
Trust (203151/Z/16/Z) and MRC (MC_PC_17230) to the Wellcome-MRC Cambridge Stem Cell Institute. J.M. was supported by a CRUK Cambridge Cancer Centre PhD fellowship. A.H. was supported by a Wellcome Trust Junior Interdisciplinary Research Fellowship (098357/Z/12/Z) and Herchel Smith Postdoctoral Research Fellowship. This work also received support from the Novo Nordisk Foundation (NNF18CC0033666 to K.K. and NNF17OC0028730 and NNF17CC0027852 to K.B.J.), Human Frontier Science Program (LT000092/2016-L to S.H.), Basic Science Research Program (NRF2014R1A6A3A01005675 to S.H.), National Institutes of Health (ZIA BC 011763 to R.I.-B.) and Wellcome PhD stutentship (220088/Z/20/Z to F.J.E.). C.V. was supported by the Engineering and Physical Sciences Research Council (1506089). The Royal Society and European Research Council (CellFateTech; 772798) supported K.J.C. B.D.S. acknowledges funding from the Wellcome Trust (098357/Z/12/Z and 219478/Z/19/Z) and Royal Society in the form of an E. P. Abraham Research Professorship (RP। R1 180165). A.H., S.H. and B.D.S. acknowledge support from core funding to the Wellcome Trust/CRUK Gurdon Institute (203144/Z/16/Z and C6946/A24843).

\section{Author contributions}

The experiments were designed, validated and conducted by J.M., F.J.E., R.I.-B. and M.P.A., with J.M. and F.J.E. working under the supervision of M.P.A. K.K. and S.U. carried out experiments under the supervision of K.B.J. A.H. developed the deep-learning-based image segmentation and other computational image analysis pipelines to quantify cell fluorescence levels, cell shape, tissue spatial organization and
SHG images. S.H. guided the experimental design for scRNA-seq and performed analysis of the data. A.H. and S.H. were both under the supervision of B.D.S. C.V. designed and provided expertise on the 3D-printed stretcher device with input from K.J.C. A.H., R.I.-B. and K.J.C. provided insights and technical expertise on tissue mechanics and stretching experiments. K.B.J. and B.D.S. supervised parts of the study and provided expertise in the epithelial stem cell field. J.M. and M.P.A. conceived of and coordinated the project, supervised the experiments and wrote the manuscript with input from A.H. and B.D.S. All authors reviewed and edited the final manuscript. A.H., R.I.-B., K.J.C., K.B.J., B.D.S. and M.P.A. acquired the funding.

\section{Competing interests}

The authors declare no competing interests.

\section{Additional information}

Extended data is available for this paper at https://doi.org/10.1038/s41556-021-00679-w. Supplementary information The online version contains supplementary material available at https://doi.org/10.1038/s41556-021-00679-w.

Correspondence and requests for materials should be addressed to M.P.A.

Peer review information Nature Cell Biology thanks John Connelly and the other, anonymous, reviewer(s) for their contribution to the peer review of this work.

Reprints and permissions information is available at www.nature.com/reprints. 

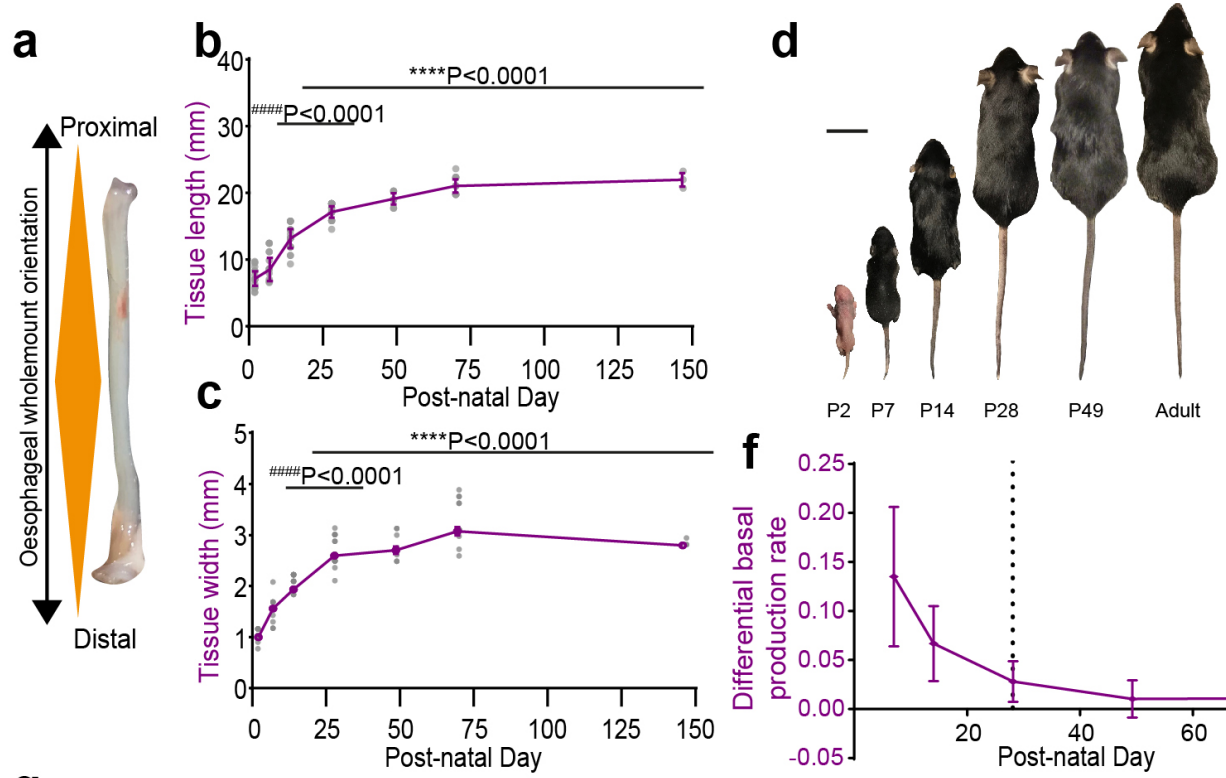

e
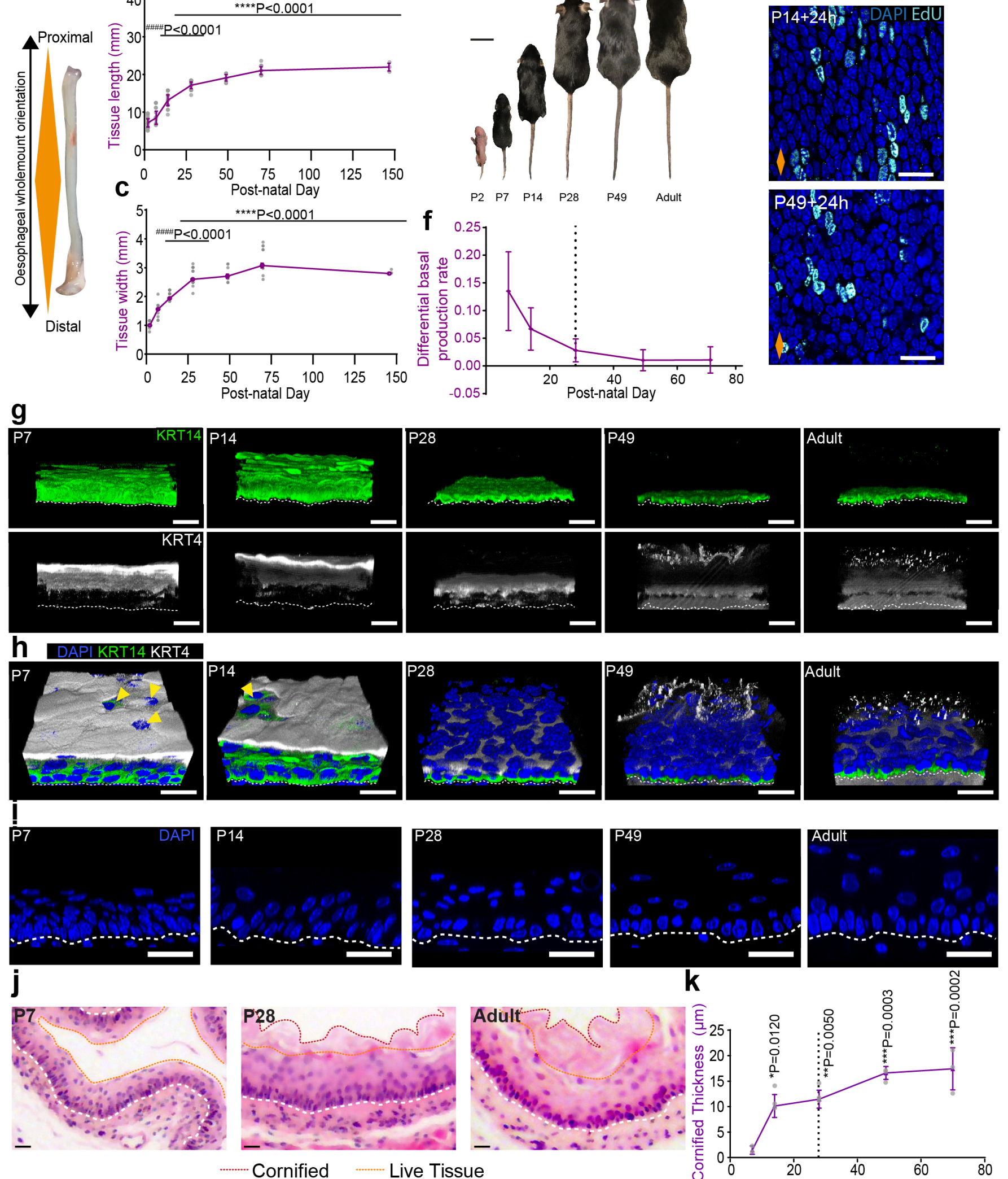

Cornified Live Tissue
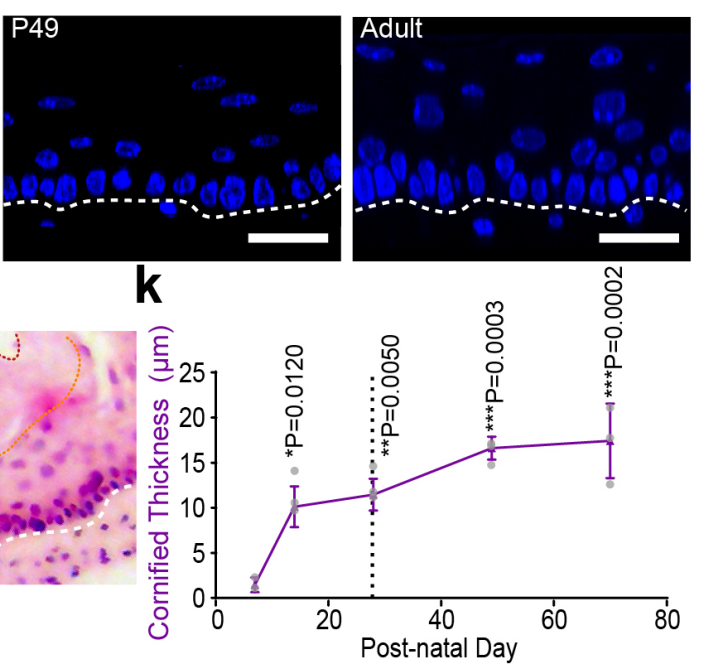

Extended Data Fig. 1 | See next page for caption. 


\section{NATURE CELL BIOLOGY}

Extended Data Fig. 1 | Postnatal characterization. a, Diagram illustrating longitudinal oesophageal orientation from proximal to distal as marked by orange diamond. $\mathbf{b}$, and $\mathbf{c}$, Oesophageal tissue growth in length and width over time, respectively. Data expressed as mean \pm SEM and analysed using one-way ANOVA with Tukey's multiple comparisons test $\left(n=103\right.$ mice; \#\#\#\#P<0.0001 relative to $P 70 ;{ }^{\star \star \star \star} P<0.0001$ relative to $\left.P 7\right)$. d, Images showing animal body growth throughout postnatal development. e, Representative images showing EdU+ basal cells 24 hours post-labelling in P14 and P49 from Fig. 1 -g. f, Graphical representation of differential basal cell production rate throughout postnatal development. See Methods; data expressed as mean $\pm \mathrm{SEM}$; $\mathrm{n}=3$ mice. g, 3D rendered z-stacks showing split confocal channels from Fig. 1i. h, Typical 3D rendered confocal z-stacks showing tilted side views from Fig. 1i. Yellow arrows indicate immature epithelial barrier. $\mathbf{i}$, Representative side views of confocal z-stacks showing the thickening of the oesophageal epithelium (OE). j, Representative H\&E sections of the oesophagus showing increasing cornification, as delimited by dotted lines. $\mathbf{k}$, Quantification of the cornified thickness. $\mathrm{n}=3$ mice; Micrometer, $\mu \mathrm{m}$. Data expressed as mean $\pm \mathrm{SEM}$ and analysed using one-way ANOVA with Tukey's multiple comparisons test $\left({ }^{\star} P<0.05,{ }^{\star \star} P<0.01,{ }^{\star \star \star} p<0.001\right.$ relative to P7). Scale bars. S1d $(2 \mathrm{~cm}) ; \mathrm{S} 1 \mathrm{e}, \mathrm{g}-\mathrm{j}(20 \mu \mathrm{m})$. Stainings. Blue, DAPI; cyan, EdU; green, KRT14; greyscale, KRT4. All data derived from wild-type C57BL/6J mice. Dashed lines indicate basement membrane. Dotted lines in graphs indicate P28. Orange diamonds depict longitudinal orientation of the oesophagus where indicated. Source data are provided. 
a
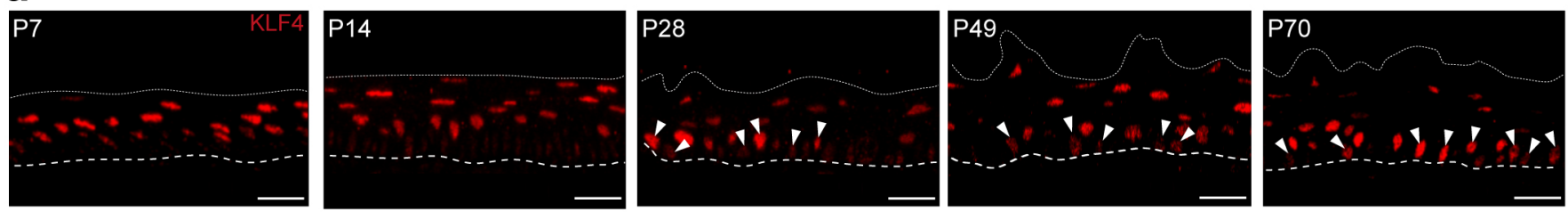

b

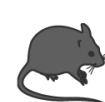

P7
P28

Adult

C

G1 G1S S/G2/M

d

e
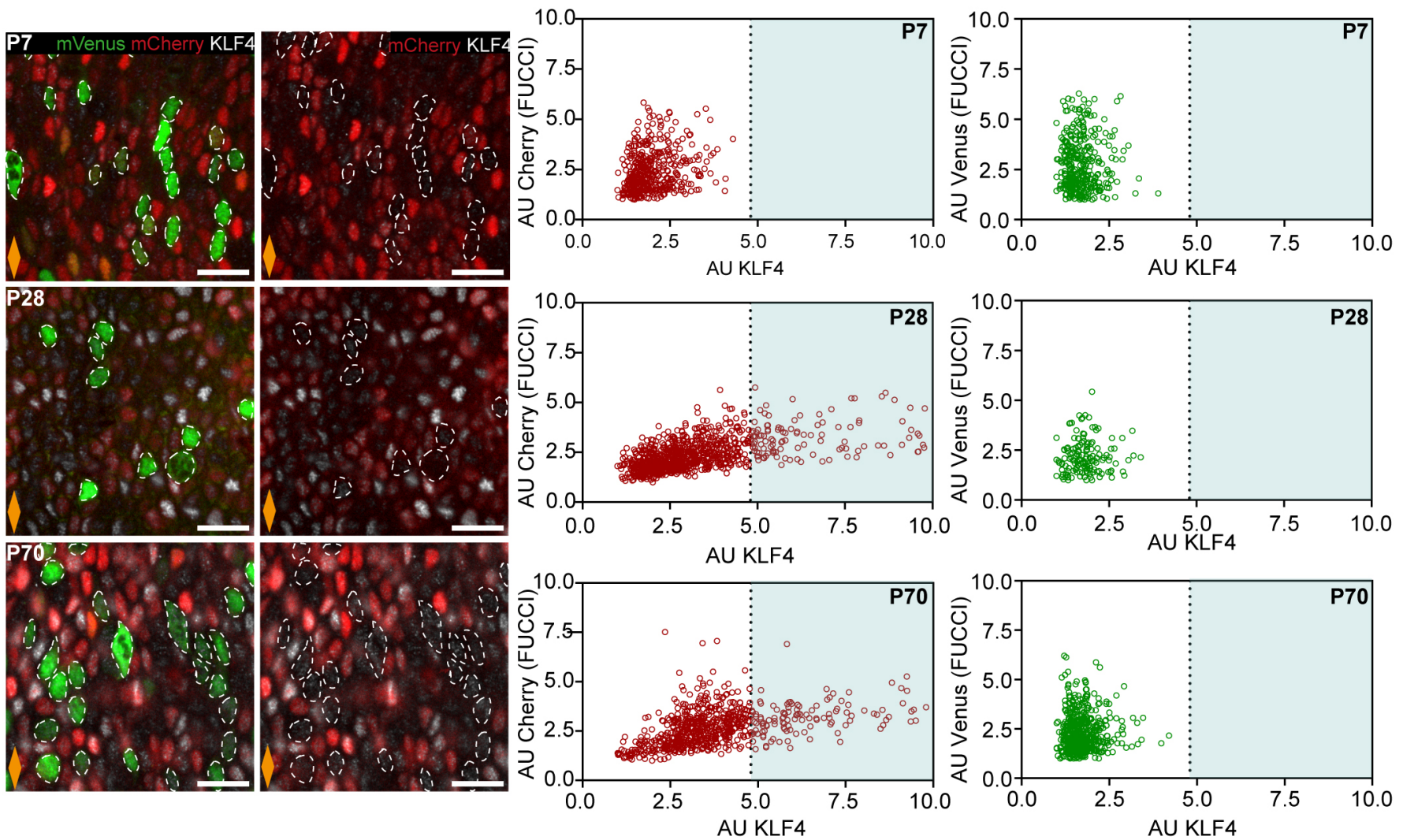

Extended Data Fig. 2 | KLF4 basal cell prolife in FUCCI2a mice. a, Representative confocal z-stacks showing side views of OE wholemounts from Fig. 2a. Dashed lines indicate basement membrane; dotted lines mark the upper limit of the OE; white arrows indicate basal KLF4+ cells. Red, KLF4. b, In vivo protocol. Oesophagi from FUCCI2a mice were collected at time points indicated. Schematic indicating expression pattern of fluorescent proteins in FUCCI2a mouse model. c, Confocal images showing basal views of typical FUCCI2a OE wholemounts in (b). Orange diamonds indicate longitudinal orientation of the oesophagus. White dashed lines indicate mVenus+ cells; green, mVenus; red, mCherry; greyscale, KLF4. $\mathbf{d}$ and $\mathbf{e}$, Correlation between KLF4 protein expression and reporter fluorescent proteins mCherry (d)/mVenus (e) in the basal layer from (b) and (c). $n=3$ mice; Scale bars $20 \mu m$. Parts of (b) were drawn by using and/or adapting diagrams from Servier Medical Art. Source data are provided. 

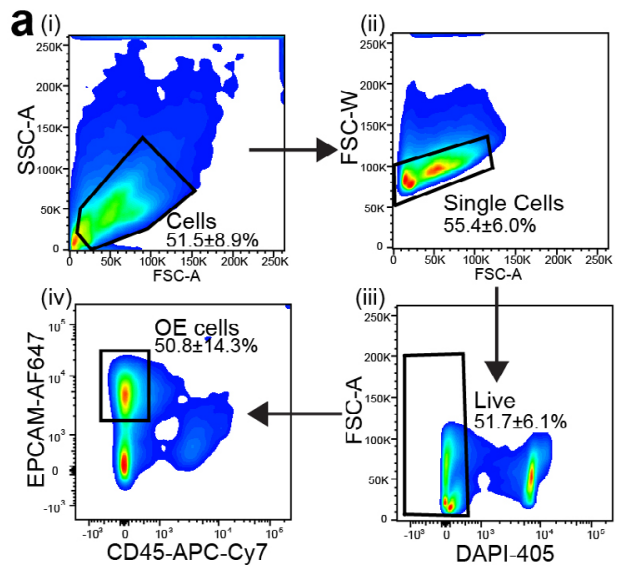

b

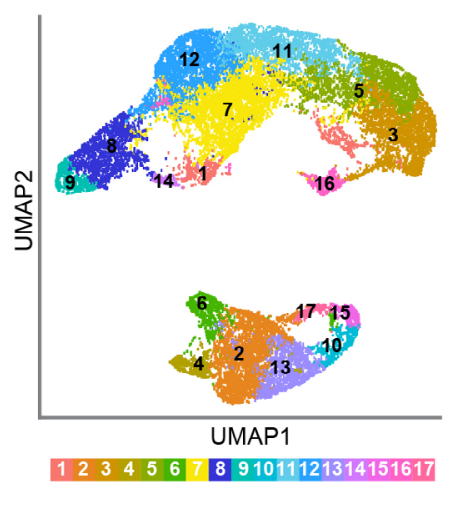

C

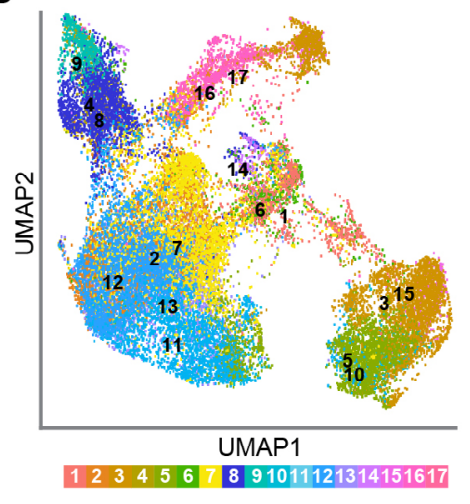

d

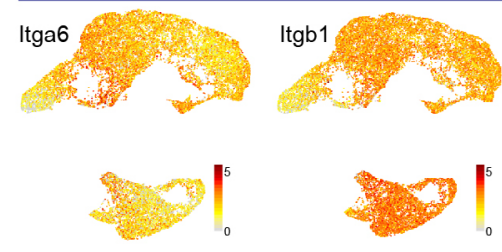

e

C Cycling Basal Resting Basal Differentiated Cluster \begin{tabular}{lllllllllllllllll}
\hline 11 & 13 & 5 & 10 & 16 & 17 & 3 & 15 & 2 & 6 & 1 & 12 & 14 & 8 & 4 & 9 & $\downarrow$
\end{tabular}

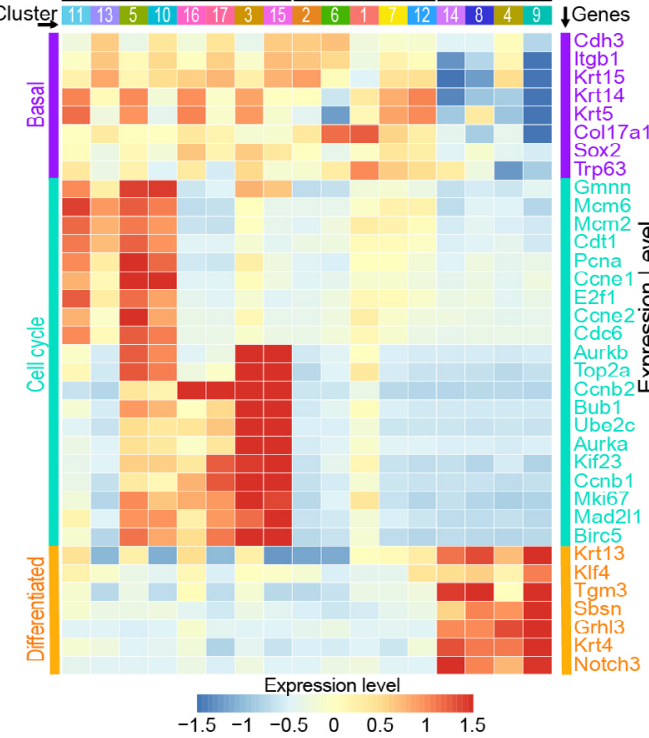

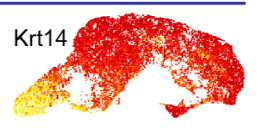

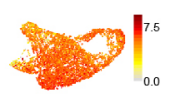

f

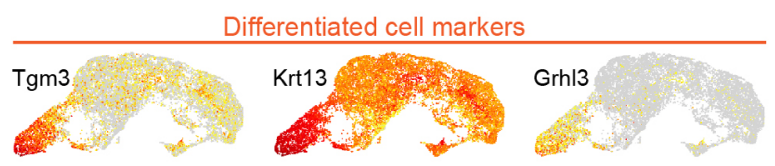

Differentiated cell markers
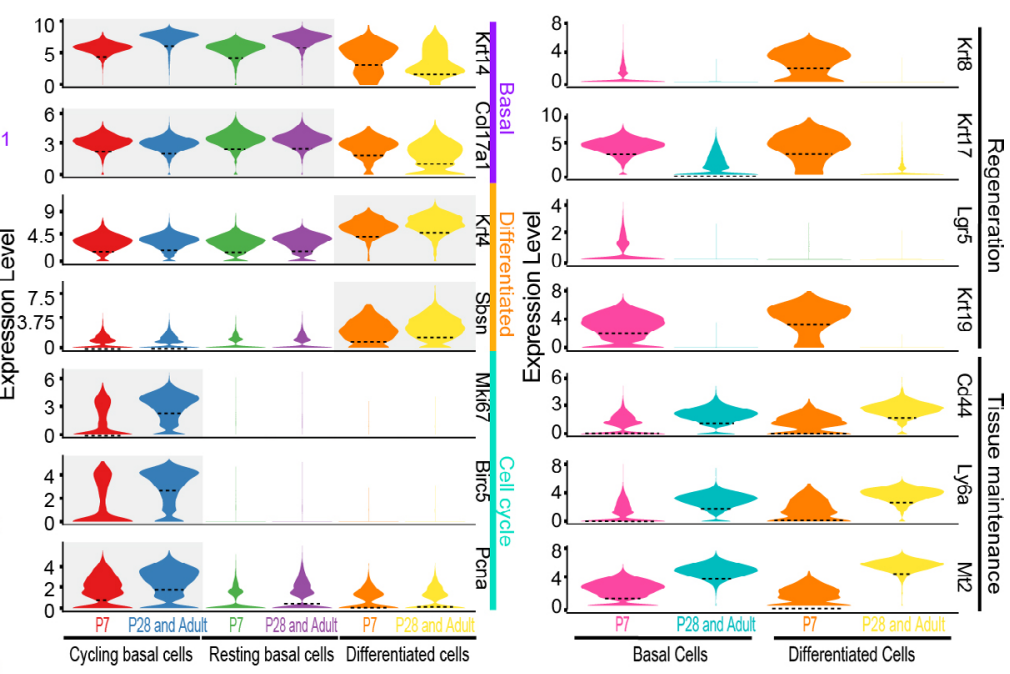

h

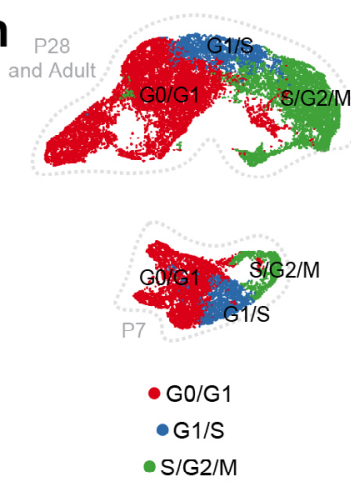

i

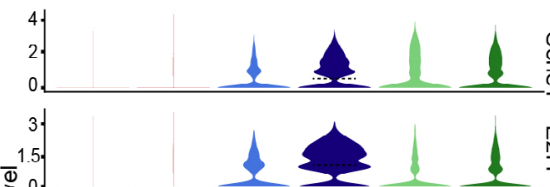

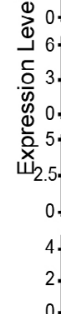

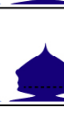

$+$

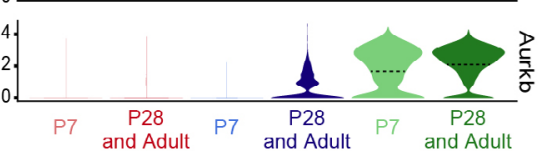

$\mathbf{j}$

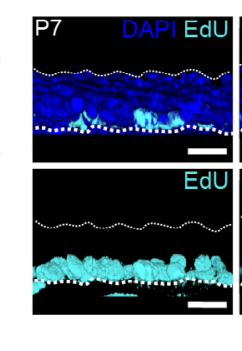

\section{g}
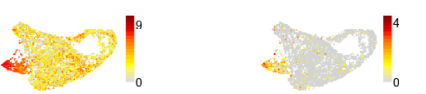
Extended Data Fig. 3 | Single-cell RNA sequencing annotation. a, Flow cytometry gating strategy for isolation of OE cells. Oesophageal cell suspensions (i) were gated to sort the single (ii) viable (iii) population, enriched for epithelial cells (iv; EpCam+/CD45-). Cells were isolated from 15 mice (P7), 12 mice (P28), and 9 (adults). Representative plots from adult sample are shown. b, UMAP representing cell clusters based on louvain clustering. c, UMAP representing the distribution of cell clusters in (b) after integrating data from different time points (see Methods). d, UMAP showing expression of representative makers for basal (left panel) and differentiated cells (right panel) in OE. e, Heatmap showing expression of representative marker genes for basal cells, cell cycle, and differentiation for the 17 clusters shown in (b in lower bar). Expression values were log2-transformed normalized UMIs followed by scaling and averaging across cells in the same clusters. f, Violin plots showing expression of representative epithelial (basal vs. differentiated) and cell cycle markers at different postnatal stages split by annotated cell cohorts in Fig. 3c. g, Violin plots showing expression of genes associated with regeneration and homeostasis for basal and differentiated cell types at distinct postnatal stages. Basal cells include both cycling and resting cells. $\mathbf{h}$, UMAP showing spatial distribution of distinct cell cycle phases, annotated using cell cycle analysis by R package scran ( $\mathrm{v}$ 1.12.1) combined with manual curation based on genes in (e). $\mathbf{i}$, Violin plots showing expression of representative cell cycle genes at postnatal stages split by cell cycle cohorts identified in (h). Colour scheme for cell cycle phases as in (h). $\mathbf{j}$, In vivo protocol. Mice were treated with EdU 2 hours prior culling at indicated time points. $\mathbf{k}$, Typical 3D rendered confocal side views showing basal EdU population (see Methods). Dashed white lines, basement membrane. Dotted white lines, upper OE limit. Blue, DAPI; cyan, EdU; scale bar $10 \mu \mathrm{m}$. For violin plots in (f), (g) and (i), expression level represents log2-transformed normalized UMIs, dotted lines indicate the median of the distribution. Colour bars of UMAPs in (d) denotes expression range. Parts of (j) were drawn by using and/or adapting diagrams from Servier Medical Art. 


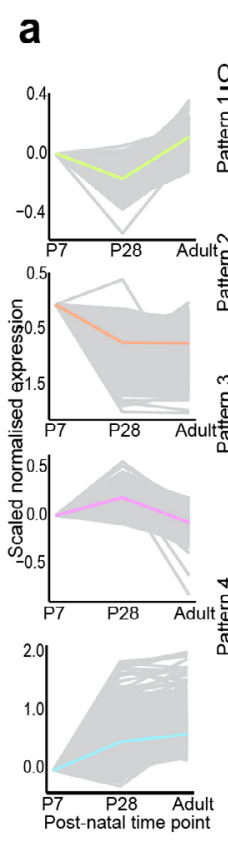

b
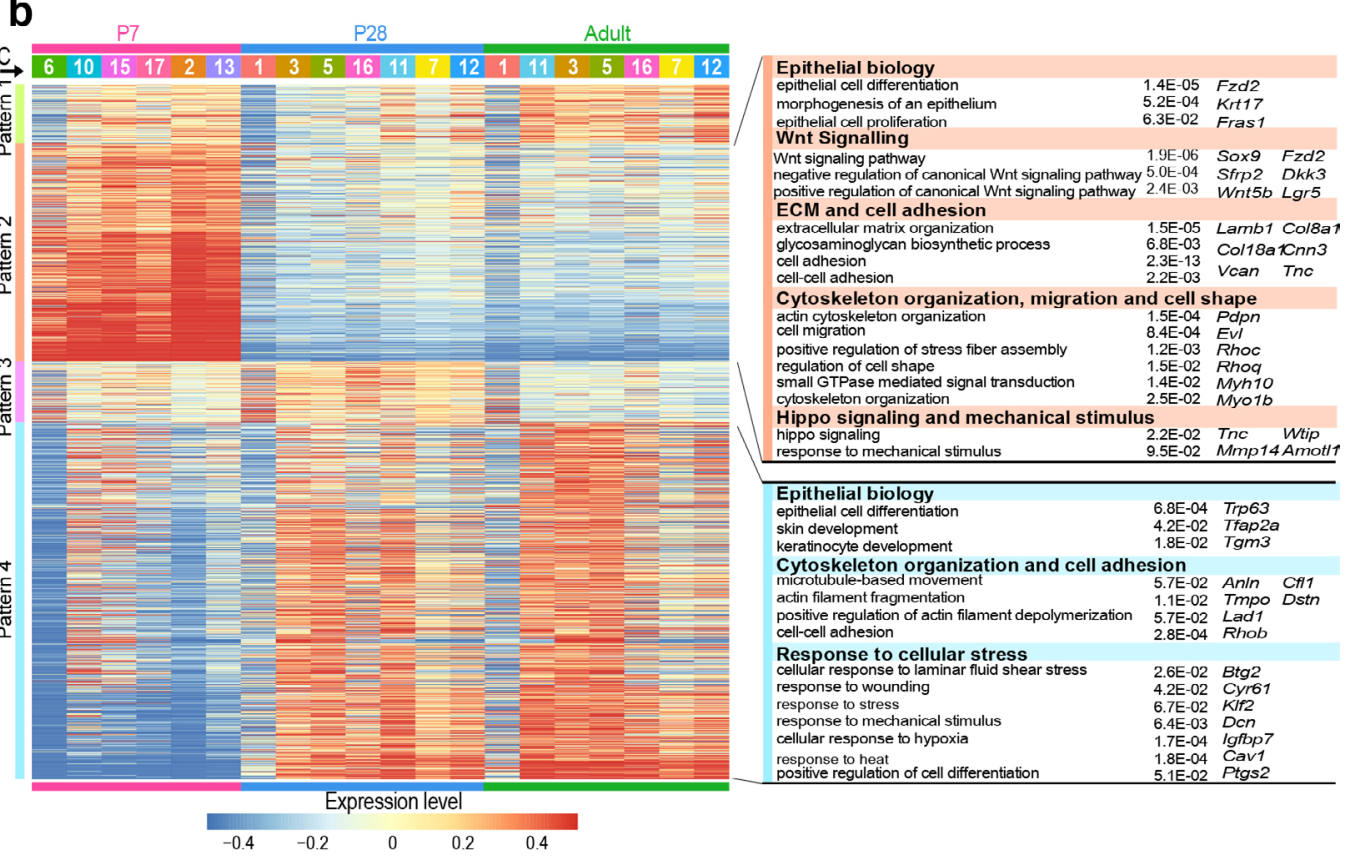

\begin{tabular}{llll} 
hippo signaling & $2.2 E-02$ & TnC Wtip \\
response to mechanical stimulus & $9.5 E-02$ & Mmp14 Amot/1 \\
\hline
\end{tabular}

\begin{tabular}{lll}
\hline Epithelial biology & & \\
epithelial cell differentiation & $6.8 \mathrm{E}-04$ & $\operatorname{Trp} 63$ \\
skin development & $4.2 \mathrm{E}-02$ & $\operatorname{Tfap2a}$ \\
keratinocyte development & $1.8 \mathrm{E}-02$ & $\operatorname{Tgm} 3$ \\
\hline
\end{tabular}

skin development

$\begin{array}{lrlll}\text { Cytoskeleton organization and cell adhesion } & & \\ \text { microtubule-based movement } & \text { 5.7E-02 } & \text { An/n } & \text { Cf1 } \\ \text { actin filament fragmentation } & 1.1 \mathrm{E}-02 & \text { Tmpo } & \text { Dstn }\end{array}$

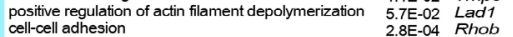

$\begin{aligned} & \text { Response to cellular stress } \\ & \text { cellular response to laminar fluid shear stress }\end{aligned} \quad 2.6 \mathrm{E}-02 \quad \mathrm{Btg} 2$ $\begin{array}{lll}\text { cellular response la laminar fuld shear stress } & 2.6 \mathrm{E}-02 & \mathrm{Btg} 2 \\ \mathrm{response} & 4.2 \mathrm{E}-02 & \mathrm{Cyr} 61\end{array}$ esponse to stress

response to mechanical stimulus

response to heat
positive regulation of cell differentiation

$\begin{array}{ll}6.7 \mathrm{E}-02 & \mathrm{~K} / 1 \mathrm{r} 2 \\ 6.4 \mathrm{E}-03 & \mathrm{Dcn}\end{array}$ \begin{tabular}{ll}
$1.8 \mathrm{E}-04$ & Cav1 \\
5 & $\mathrm{E}-02$ \\
\hline
\end{tabular}
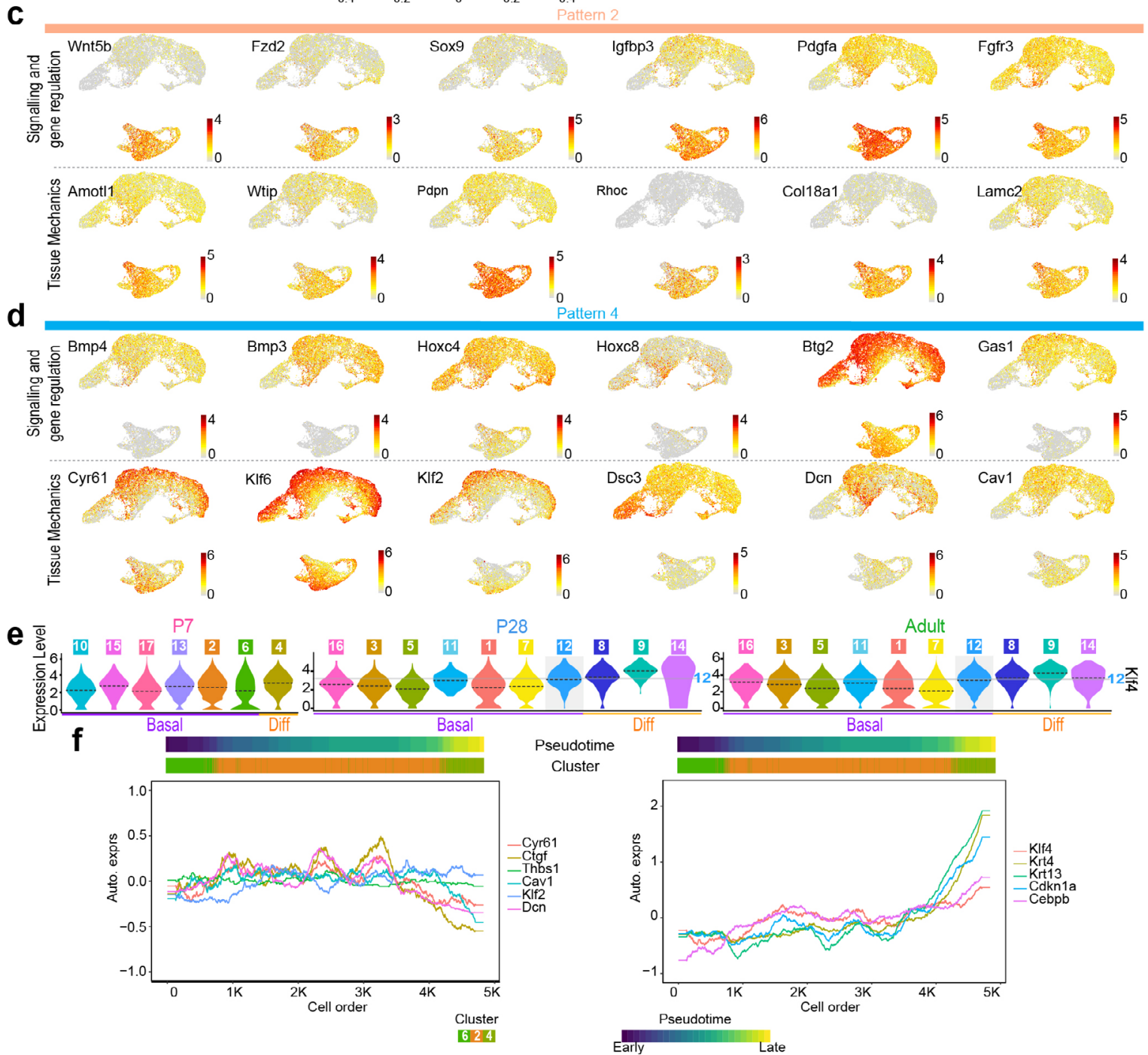

Extended Data Fig. 4 | See next page for caption. 
Extended Data Fig. 4 | Single-cell RNA sequencing expression profile. a, Distinctive patterns of gene expression in basal cells as defined in (Fig. 3c). Grey, relative expression profiles of individual genes belonging to each pattern. Solid coloured lines, median values at each time point. To calculate the relative expression profiles, log2-transformed normalized UMIs were scaled and averaged across all basal cells at each time point and adjusted compared to the value at P7. b. Heatmap representing expression of individual genes belonging to the 4 patterns in (a). For expression values, log2-transformed normalised UMIs were scaled and averaged across all basal cells for each cluster and time point. The table on the right shows selected GO terms for major Pattern 2 and Pattern 4, corresponding p-values and representative genes. Closely related GO terms are grouped together. See Supplementary Table 3 for GO analysis result for all 4 expression patterns. $\mathbf{c}$ and $\mathbf{d}$, UMAPs showing expression of genes related to key biological processes from Gene Ontology analysis for Patterns 2 (c) and 4 (d) in (b). e, Violin plots showing expression of KIf4 in cells of individual clusters at P7 (left), P28 (middle) and Adult (right). f, Expression of relevant genes along the pseudotime trajectory from basal resting to differentiated cells for P7. Left panel, YAP target genes (Cyr61, Ctgf, Thbs1) and genes associated with a response to mechanical stimuli (Cav1, KIf2, Dcn). Right panel, depicts KLF4 target genes (Krt4, Krt13, Cdnk1a, Cebpb). For violin plots in (e), expression level means log2-transformed normalized UMIs and dotted lines indicate the median of the distribution. Colour bars of UMAPs in (c) and (d) indicate log2-transformed normalized UMIs. Gene expression in (f) is represented as auto-scaled, log2-transformed normalized UMIs smoothed using a rolling mean along its trajectory with a window size of $5 \%$ of cells. Two bars on the top denotes the arrangement of cells according to pseudotime and clusters in Extended Data Fig. 3b, respectively. 
a

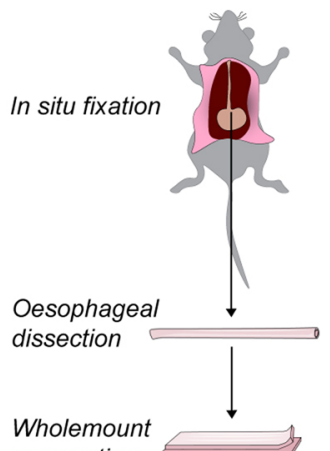

preperation b

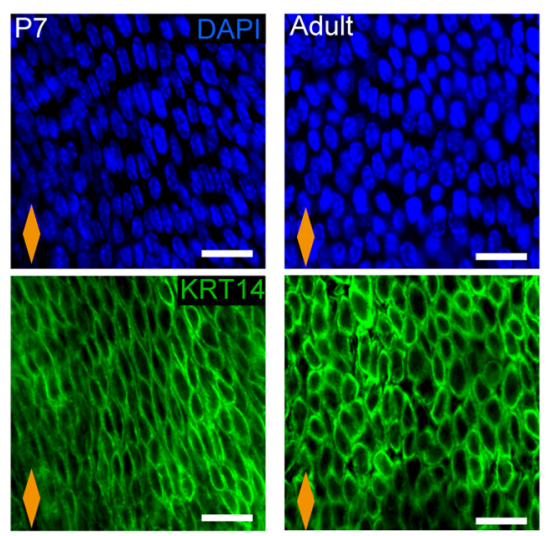

d
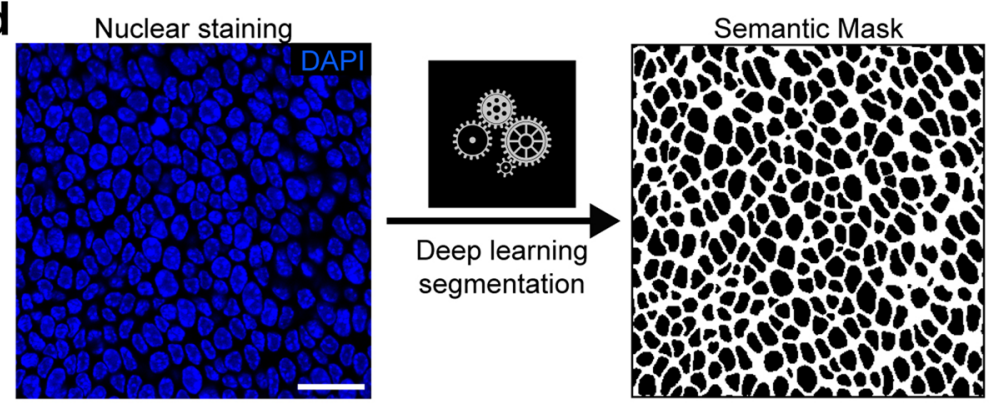

C

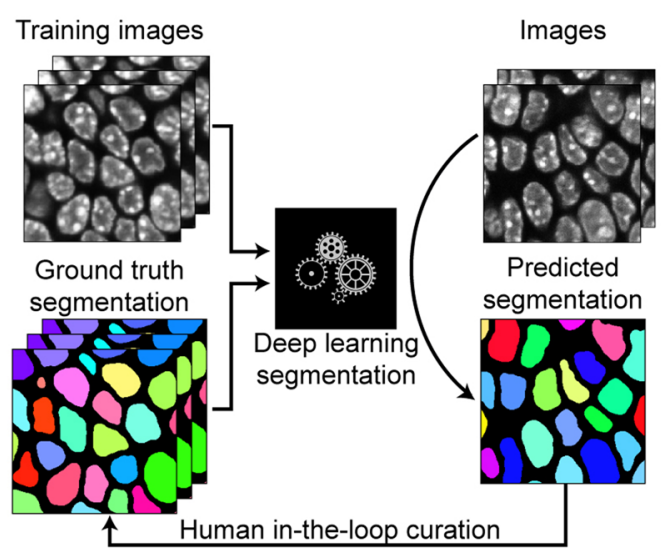

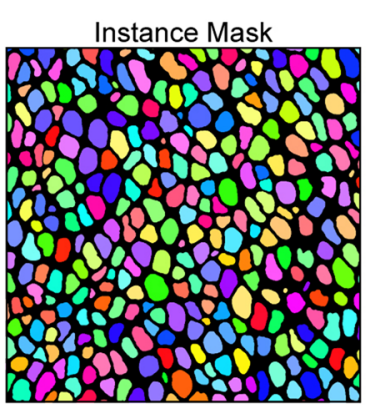

Mask overlay with original image

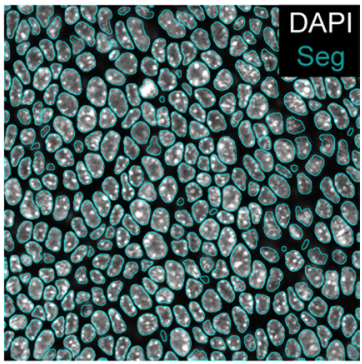

e

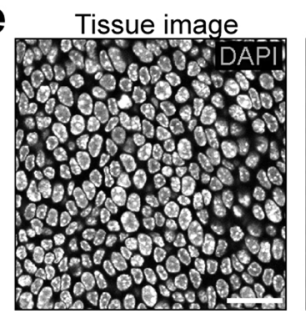

Semantic Mask

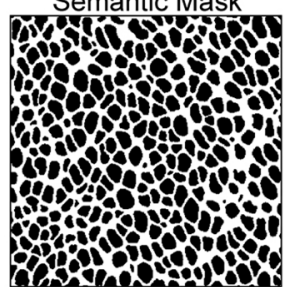

Cell centroids

f

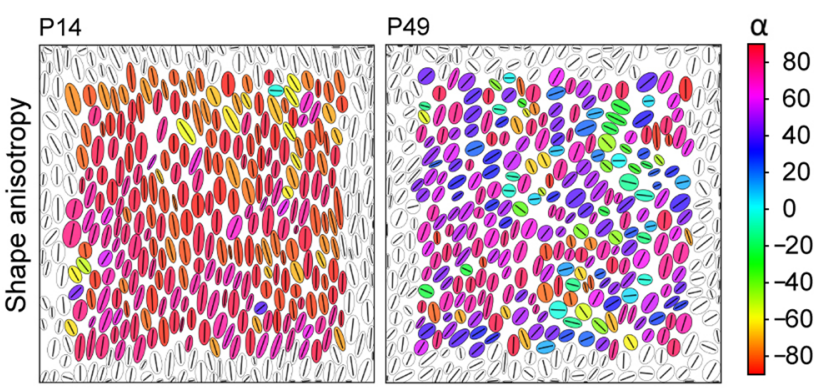

g

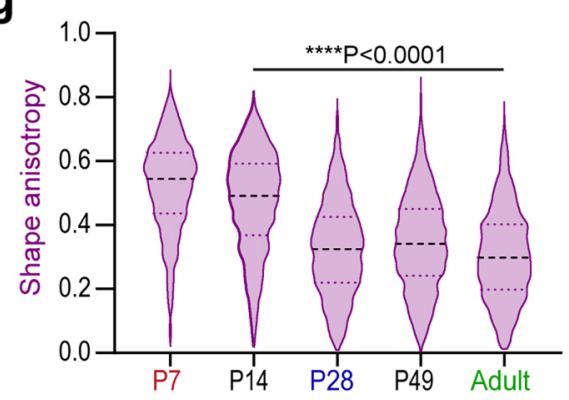

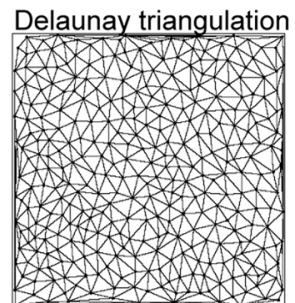

Voronoi diagram

$\mathbf{h}_{\mathrm{P} 14}$

s.

P49

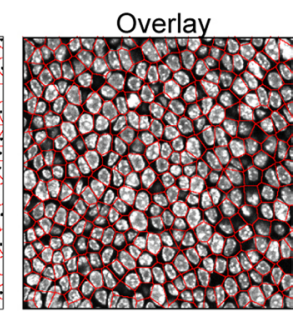

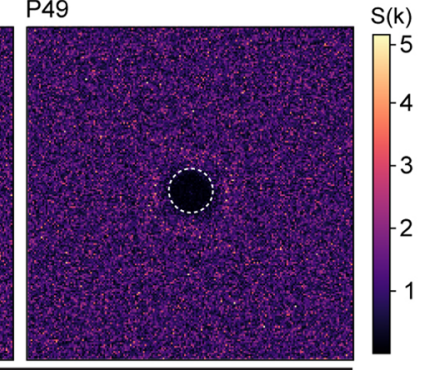

$k_{x} l_{c} / 2 \pi$

i

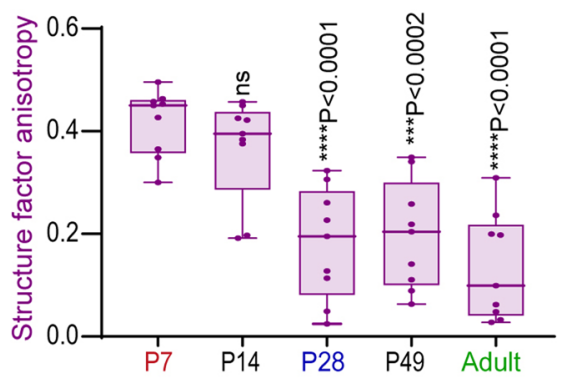

Extended Data Fig. 5 | See next page for caption. 
Extended Data Fig. 5 | Deep Learning based segmentation. a, Protocol for in situ fixation of the oesophagus, and b, Images from in situ oesophagi compared with oesophagi fixed immediately after dissection confirm that basal cell shape was not affected by tissue harvesting (Supplementary to Fig. 4a). Blue, DAPI; green, KRT14. Scale bar, $20 \mu \mathrm{m}$. c, Schematic depicting deep learning based segmentation principle. Manually or semi-automatically annotated 'ground truth' images were used to train a U-Net convolutional neural network. Training of the network was assessed on validation images and iteratively optimized until the achievement of satisfactory automated segmentation. $\mathbf{d}$, Schematic of pipeline utilised for segmentation of single z-slice confocal images of OE basal layer. Nuclear segmentation was based on DAPI staining (blue). Mask overlay shows the match between the binary mask and the original fluorescence image. Scale bar, $20 \mu \mathrm{m}$. e, Schematic describing the computation of Voronoi diagrams of the tissue. Single z-slice confocal images of the OE basal layer are segmented as described in (d). Cell centroids are computed using the binary mask. Delaunay triangulation of cells was performed using cell centroid coordinates. Voronoi diagrams are calculated as the dual of Delaunay triangulation of cells in the tissue and overlaid onto the original fluorescence image. Scale bar, $20 \mu \mathrm{m}$. f, Cell shape anisotropy tensor at P14 and P49 (supplementary to Fig. 4d). n=3 mice. Orientation is colour-coded. Results from a representative experiment are shown; $n=3$ mice. $\mathbf{g}$, Violin plots showing the distribution in cell shape anisotropy throughout postnatal development. $n=2052-2594$ cells from 3 animals per time point. Black dashed line, median. One-way ANOVA with Tukey's multiple comparisons test ( ${ }^{\star \star \star \star} P<0.0001$ relative to P7). h, Bidimensional structure factor at P14 and P49 (supplementary to Fig. 4e). Changes in the dashed white outline (from ellipse to circle) depict a transition from anisotropic to isotropic spatial distribution over time; $n=3$ mice. i, Structure factor shape anisotropy distribution as shown in (h) and Fig. 4e. Box plots show median and quartiles; and whiskers, minima/maxima. Individual measurements from $n=3$ mice; (ns, not significant; ${ }^{\star \star \star} P<0.001,{ }^{\star \star \star \star} P<0.0001$ relative to P7). All data derived from wild-type C57BL/6J mice. Parts of (a) were drawn by using and/or adapting diagrams from Servier Medical Art. Source data are provided. 
a

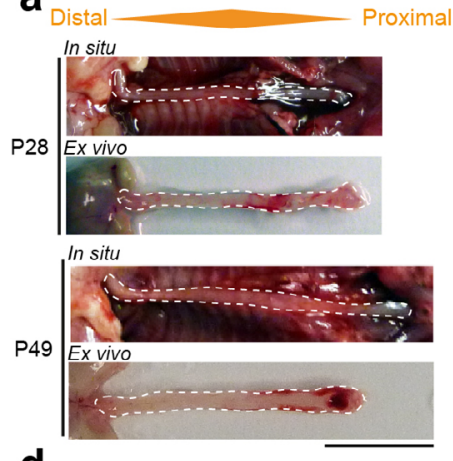

d

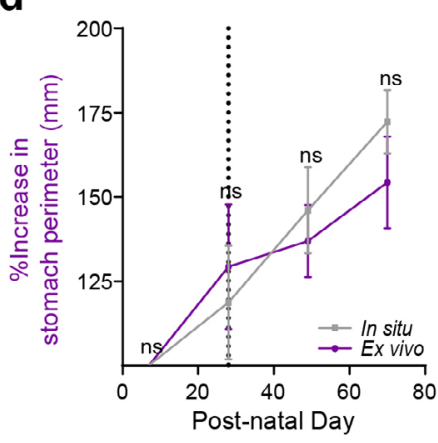

b
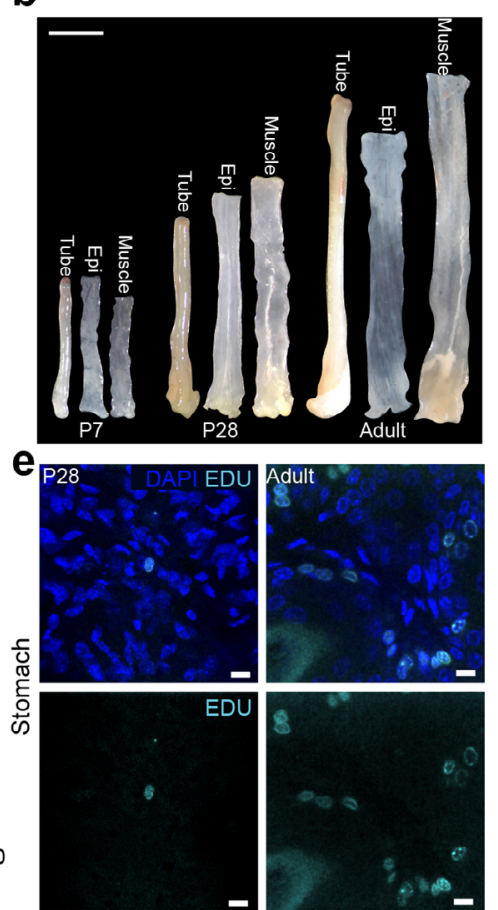

C

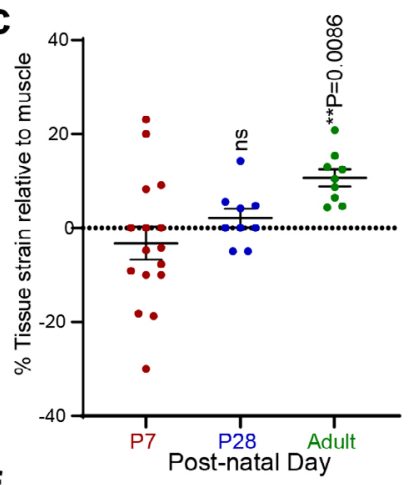

$f$

Orientation map

h

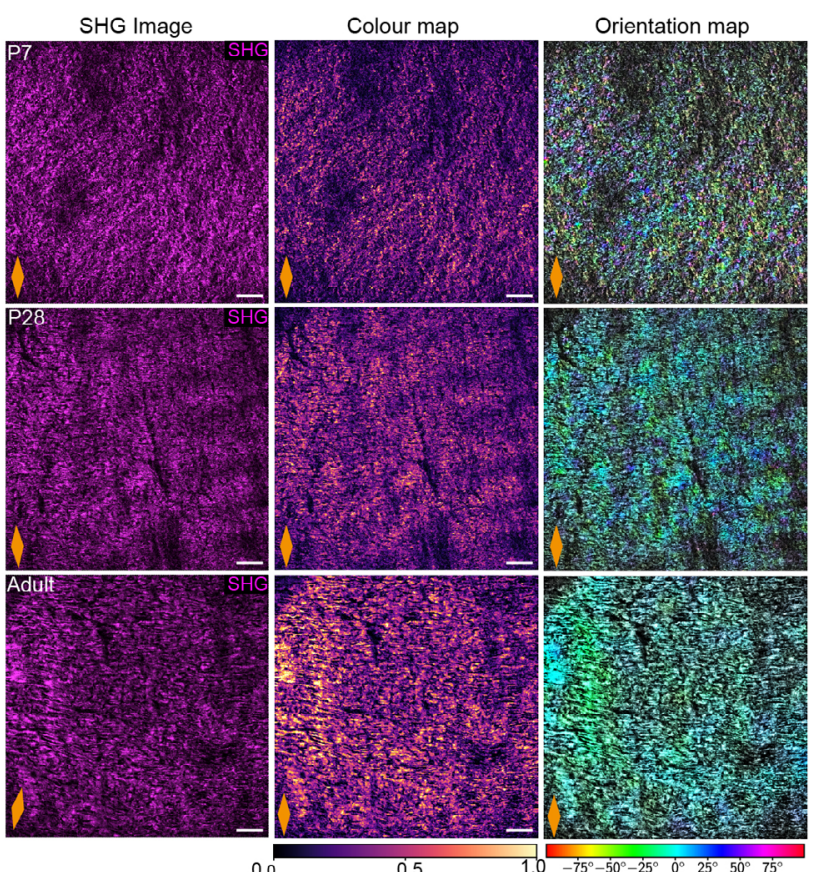

i

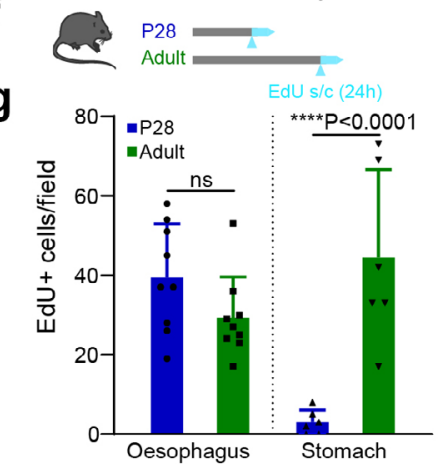

Orientation histogram
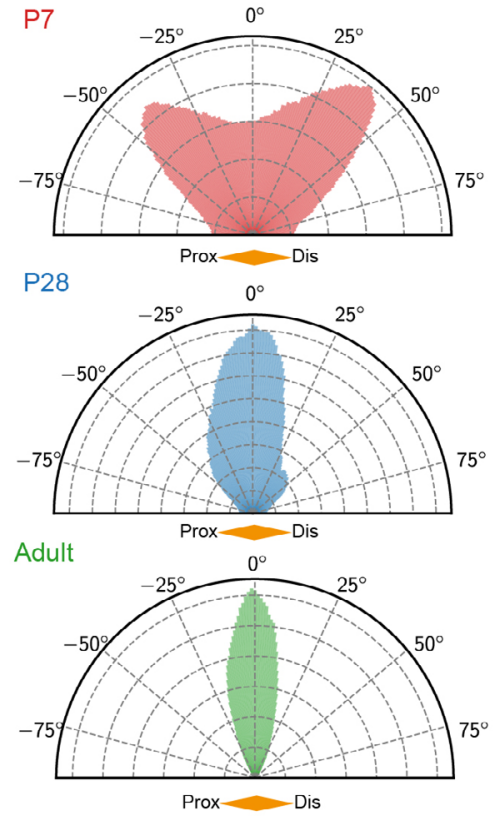

j
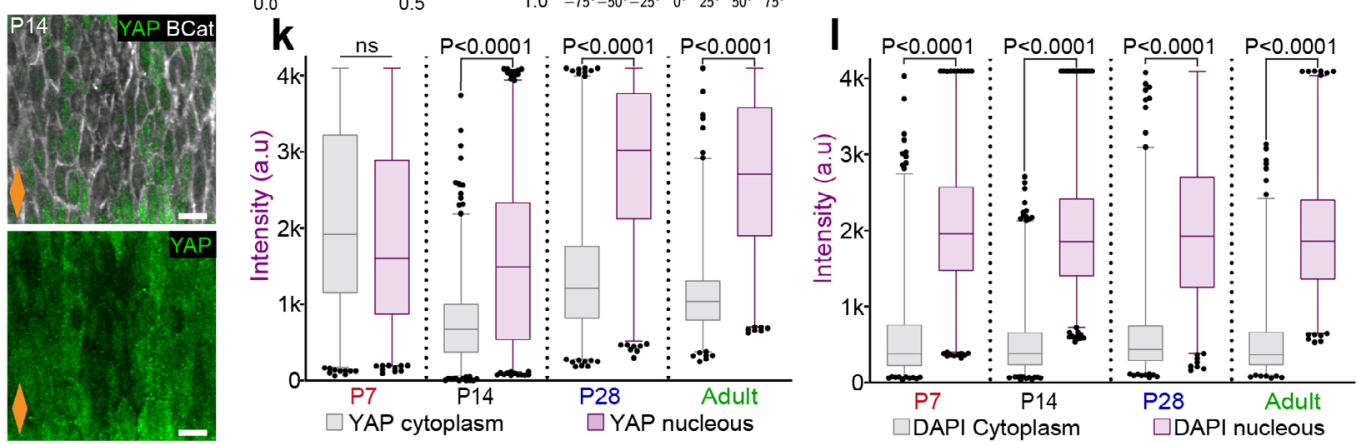

Extended Data Fig. 6 | See next page for caption. 
Extended Data Fig. 6 | Oesophageal tissue strain and second harmonic generation. a, In situ and immediate ex vivo images of oesophageal tubes at P28 and P49 (supplementary to Fig. 5b). White dashed lines delineate oesophageal tube; scale bar $1 \mathrm{~cm}$. b. Representative images showing the size of combined and separate oesophageal layers; full oesophageal tube (Tube), epithelial composite (Epi) and muscle layer (Muscle); scale bar 5 mm. c, Longitudinal tissue strain relative to muscle, represented as percentage. Data expressed as mean \pm SEM. One-way ANOVA with Tukey's multiple comparisons test ( $P 7 n=16, P 28$ and Adult $n=9 ;{ }^{\star \star} P<0.01$ relative to $P 7 ; n s$, not significant). d, Measure of the stomach perimeter over time. Mean values $\pm \mathrm{SEM} ; \mathrm{n}=3$ mice. Two-way ANOVA, with Tukey's multiple comparisons test ( $\mathrm{ns}$, not significant). Millimetre, mm. e, Basal confocal view of EdU+ cells (cyan) in wholemounts of typical squamous stomach epithelium from (f; Blue, DAPI). f, In vivo protocol. Mice were treated with a single EdU injection $24 \mathrm{~h}$ prior stomach collection at the time points indicated. $\mathbf{g}$, Quantification of EdU+ basal cells per field from (f). Presented as mean $\pm S D ; n=3$. $\mathbf{h}$, Representative views of stroma underlying OE basement membrane using second harmonic generation (SHG). Left panels, collagen in magenta. Middle panels, colour map of SHG signal intensity. Right panels, colour-coded local orientation map of SHG signal. Scale bar $100 \mu$ m. $\mathbf{i}$, Representative histograms depicting orientation distribution of collagen fibres in (h). $n=3$ mice. j, Basal view of representative OE wholemounts at P14. Green, YAP; greyscale, B-Catenin (BCat). Scale bar $10 \mu \mathrm{m}$. $\mathbf{k}$ and $\mathbf{I}$, Quantification of basal nuclear and cytoplasmic staining of YAP and DAPI, respectively (see Methods; supplementary to Fig. $5 \mathrm{~m}$ ). A total of 20 cells for 3 different animals were measured. All data derived from wild-type C57BL/6J mice. $\mathbf{g}$, $\mathbf{k}$ and $\mathbf{I}$ was performed using two-tailed unpaired t test ( ${ }^{\star \star \star \star} P<0.0001$; ns, not significant). Box plots show median and quartiles; whiskers, 0.1 and 0.99 percentiles. Orange diamonds depict longitudinal orientation of the oesophagus where indicated. Parts of (f) were drawn by using and/or adapting diagrams from Servier Medical Art. Source data are provided.Source data 

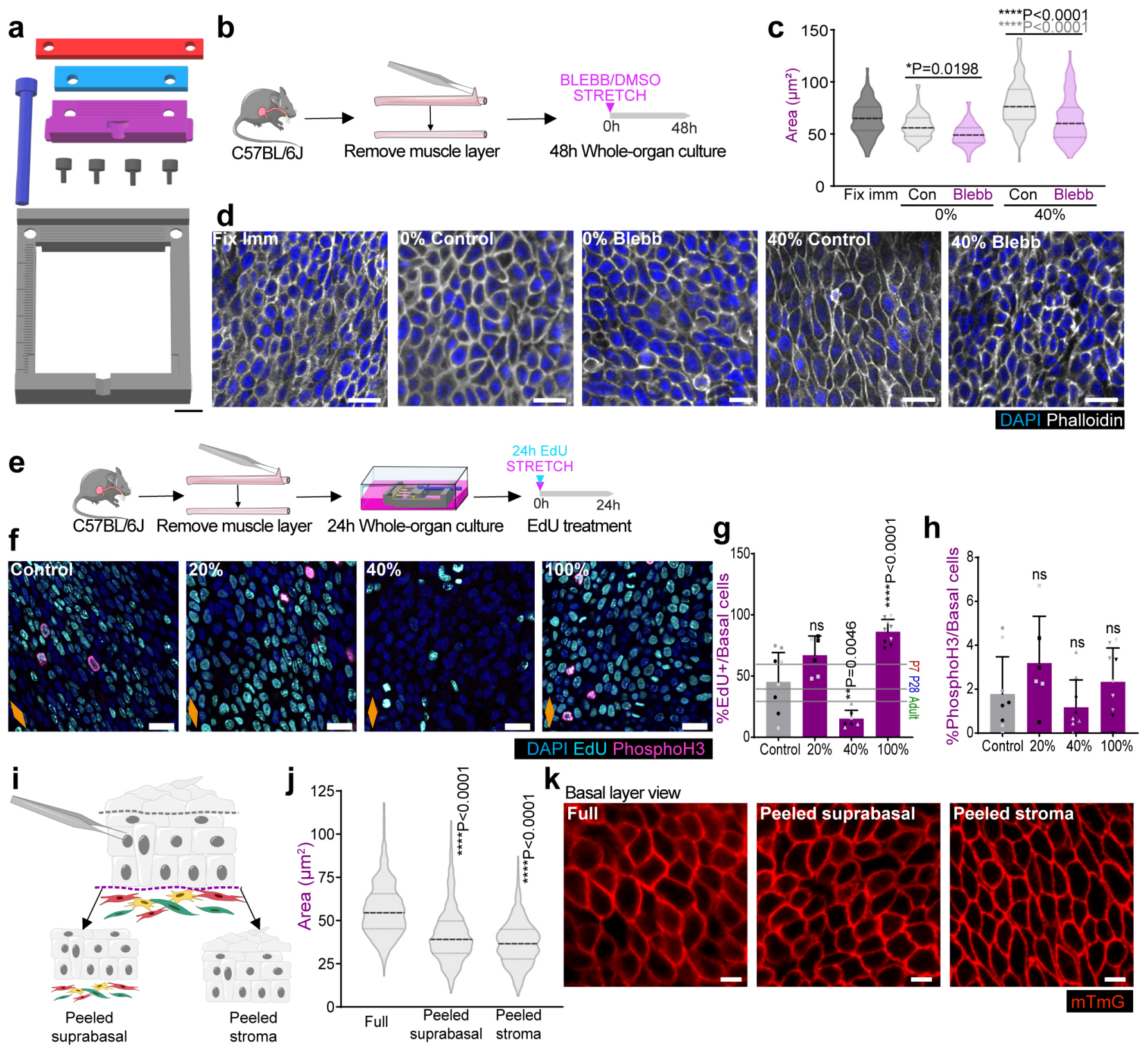

$\mathbf{K}_{\text {Basal layer view }}$

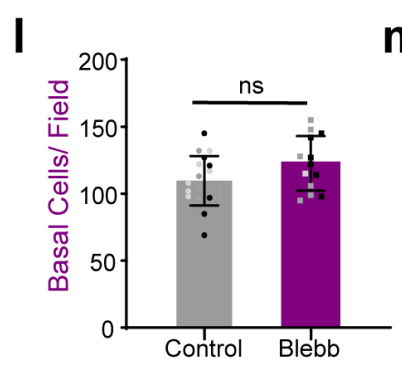

m

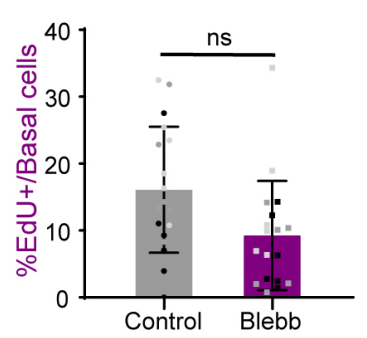

Extended Data Fig. 7 | See next page for caption. 
Extended Data Fig. 7 | Changes in tissue mechanics at cellular level. a, Model of parts required for 3D printed stretcher. Scale bar, $1 \mathrm{~cm}$. $\mathbf{b}$, In vitro protocol. Oesophagi were exposed to $40 \%$ stretch using stretcher (Fig. $7 a$ ) and treated with/without $25 \mu \mathrm{m}$ blebbistatin (BLEBB) for 48 hours. c, Individual basal cell areas. Data analysis was performed using Two-way ANOVA with Tukey's multiple comparisons test $\left(n=3\right.$ mice; ${ }^{\star} P<0.05,{ }^{\star \star \star \star \star} P<0.0001 ; n s$, not significant; black indicates significance between control vs. BLEBB conditions; grey indicates statistical differences between stretching conditions). d, Confocal basal views of typical organ cultures after 48 hour BLEBB treatment in vitro from (b). Blue represents DAPI, greyscale shows phalloidin staining. Scale bar, $20 \mu \mathrm{m}$. e, In vitro protocol. Oesophagi stretched at the indicated levels, treated with EdU for 1 hour, and kept in vitro as whole-organ cultures for a 24 hours. f, Confocal basal views showing EdU (cyan) and phosphohistone H3 (PhosphoH3 in magenta) staining in cultures from (e). Scale bar, $20 \mu \mathrm{m}$. $\mathbf{g}$ and $\mathbf{h}$, Basal quantification of EdU+ and PhosphoH3+ cells expressed as percentage of DAPI+ cells from (e). Mean \pm SD One-way ANOVA with Tukey's multiple comparisons test ( $n=3$ mice; ${ }^{\star \star} P<0.01,{ }^{\star \star \star \star} P<0.0001$ relative to Control; $n s$, not significant). $\mathbf{i}$, In vivo protocol. Oesophageal wholemounts from Rosa26-mT/mG mice were mechanically peeled, separating cornified suprabasal layers or underlying stroma (see Methods). $\mathbf{j}$, Individual area of basal cells. Data analysis was performed using One-way ANOVA with Tukey's multiple comparisons test ( $n=3$ mice; ${ }^{\star \star \star \star} P<0.0001$ relative to full tissue). $\mathbf{k}$, Confocal basal views of typical OE wholemounts after mechanical separation. Red, mTmG; scale bar, $10 \mu \mathrm{m}$. I, Basal cell density, expressed in number of cells per field after BLEBB treatment in Fig. 7e. Mean \pm SD; $n=3$ mice. Unpaired t test ( $n s$, not significant). $\mathbf{m}$, Basal quantification of EdU+ cells expressed as percentage of DAPI+ cells after BLEBB treatment in Fig. 7e. Mean $\pm \mathrm{SD} ; \mathrm{n}=3$ mice. Unpaired t test (ns, not significant). Data derived from wild-type C57BL/6J mice, unless otherwise stated. Individual points show individual measurements, greyscale indicates values from each of 3 mice. Parts of (b, e) were drawn by using and/or adapting diagrams from Servier Medical Art. Source data are provided.Source data 


\section{natureresearch}

Corresponding author(s): Maria P. Alcola

Last updated by author(s): Dec 3, 2020

\section{Reporting Summary}

Nature Research wishes to improve the reproducibility of the work that we publish. This form provides structure for consistency and transparency in reporting. For further information on Nature Research policies, see Authors \& Referees and the Editorial Policy Checklist.

\section{Statistics}

For all statistical analyses, confirm that the following items are present in the figure legend, table legend, main text, or Methods section.

n/a Confirmed

$\bigotimes$ The exact sample size $(n)$ for each experimental group/condition, given as a discrete number and unit of measurement

$\square \bigotimes$ A statement on whether measurements were taken from distinct samples or whether the same sample was measured repeatedly

The statistical test(s) used AND whether they are one- or two-sided

The statistical test(s) used AND whether they are one- or two-sided
Only common tests should be described solely by name; describe more complex techniques in the Methods section.

$\square$ A description of all covariates tested

$\square$ \ A description of any assumptions or corrections, such as tests of normality and adjustment for multiple comparisons

A full description of the statistical parameters including central tendency (e.g. means) or other basic estimates (e.g. regression coefficient)

AND variation (e.g. standard deviation) or associated estimates of uncertainty (e.g. confidence intervals)

For null hypothesis testing, the test statistic (e.g. $F, t, r$ ) with confidence intervals, effect sizes, degrees of freedom and $P$ value noted

Give $P$ values as exact values whenever suitable.

Х $\square$ For Bayesian analysis, information on the choice of priors and Markov chain Monte Carlo settings

Х $\square$ For hierarchical and complex designs, identification of the appropriate level for tests and full reporting of outcomes

$\square$ Estimates of effect sizes (e.g. Cohen's $d$, Pearson's $r$ ), indicating how they were calculated

Our web collection on statistics for biologists contains articles on many of the points above.

\section{Software and code}

Policy information about availability of computer code

Data collection LAS X 3.5.5.19976 Leica, https://www.leica-microsystems.com/products/microscope-software/p/leica-las-x-Is/ Volocity 5.3.3, PerkinElmer

Fiji, ImageJ 1.51W, https://imagej.net/Downloads

BD FACSDiva 8.0.1, https://www.bdbiosciences.com/us/instruments/clinical/software/flow-cytometry-acquisition/bd-facsdiva-software/ facsdiva-software-v-803-win-7-32-bit-os/p/659523

DAVID v6.8, https://david.ncifcrf.gov/home.jsp

R monocle v4, https://www.bioconductor.org/packages/release/bioc/html/monocle.html

Fiji, ImageJ 1.51W, https://imagej.net/Downloads

GraphPad, Prism 8, https://www.graphpad.com/scientific-software/prism/

FlowJo v10.6.2, BD, https://www.flowjo.com/solutions/flowjo/downloads

Ultimaker Cura 4.8, Ultimaker, https://ultimaker.com/software/ultimaker-cura

Fusion360, Autodesk, https://www.autodesk.co.uk/products/fusion-360

CellRanger (v3.0.2), 10x Genomics, https://support.10xgenomics.com/single-cell-gene-expression/software/downloads/latest

scran (v1.6.9), Lun et al., 2016, https://bioconductor.org/packages/release/bioc/html/scran.html

scater (1.12.2), McCarthy et al., 2017, https://bioconductor.org/packages/release/bioc/html/scater.html

igraph (v1.2.4.1), Csardi and Nepusz, 2005, https://igraph.org/r/

Seurat (v3.0.2), Stuart et al., 2019, https://satijalab.org/seurat/

pheatmap (v1.0.12), Raivo Kolde, https://cran.r-project.org/web/packages/pheatmap/

Python 3.0, Python Software Foundation, https://www.python.org/

SciPy 1.4.1, Python Software Foundation, https://www.scipy.org/

NumPy 1.18.0, Python Software Foundation, https://numpy.org/

NetworkX 2.4, NetworkX, https://networkx.github.io/ 
Keras 2.3.0, Keras, https://keras.io/

TensorFlow 2.0, TensorFlow, https://github.com/tensorflow/tensorflow

OpenCV-Python OpenCV, https://opencv.org/

Scikit-image 0.16.2, Scikit-image https://scikit-image.org/

Matplotlib 2.2.5 LTS, Matplotlib, https://matplotlib.org/

Seaborn 0.10.0, Seaborn, https://seaborn.pydata.org/

For manuscripts utilizing custom algorithms or software that are central to the research but not yet described in published literature, software must be made available to editors/reviewers. We strongly encourage code deposition in a community repository (e.g. GitHub). See the Nature Research guidelines for submitting code \& software for further information.

\section{Data}

Policy information about availability of data

All manuscripts must include a data availability statement. This statement should provide the following information, where applicable:

- Accession codes, unique identifiers, or web links for publicly available datasets

- A list of figures that have associated raw data

- A description of any restrictions on data availability

Data Availability

Single-cell RNA sequencing (scRNA-seq) data that support the findings of this study have been deposited in ArrayExpress under accession code EMTAB-8662. Source data have been provided. All other data supporting the findings of this study are available from the corresponding author on reasonable request.

Code Availability

The statistical analysis of the single-cell RNA sequencing data was performed using R codes developed for this study. Image segmentation and computational image analysis was performed using Python codes developed and/or adapted for this study. All codes for the computational analysis above are available at https:// github.com/BenSimonsLab/McGinn_Nat-Cell-Biol_2021.

\section{Field-specific reporting}

Please select the one below that is the best fit for your research. If you are not sure, read the appropriate sections before making your selection. $\bigotimes$ Life sciences $\quad \square$ Behavioural \& social sciences $\quad \square$ Ecological, evolutionary \& environmental sciences

For a reference copy of the document with all sections, see nature.com/documents/nr-reporting-summary-flat.pdf

\section{Life sciences study design}

All studies must disclose on these points even when the disclosure is negative.

Sample size

Sample size was determined from pilot studies and previous published research studies as listed below:

Doupe, D.P. et al. A single progenitor population switches behavior to maintain and repair esophageal epithelium. Science 337, 1091-1093

(2012).

Alcolea, M.P. et al. Differentiation imbalance in single oesophageal progenitor cells causes clonal immortalization and field change. Nat Cell Biol 16, 615-622 (2014).

Frede, J. et al. A single dividing cell population with imbalanced fate drives oesophageal tumour growth. Nature Cell Biology. Nature Publishing Group, 18(9), pp. 967-978. doi: 10.1038/ncb3400 (2016).

A minimum sample size of $n=3$ mice/biological replicates was used whenever possible. This is the case for all experiments, with the exception of data presented in Figure $6 f$. This allowed for replicate images to be taken per sample (minimum of 3 ), and data to be analyzed both internally within said sample, and between at least 3 biological replicates. Figure $6 \mathrm{f}$ shows the data for $\mathrm{n}=2$ due to animal availability issues associated with COVID-19 pandemic restrictions. In this unique case, the number of cells analysed per sample was maximised in order to obtain biologically meaningful data, all other criteria listed above were still applied in this case.

Data exclusions Exclusions to data were made in the case of RNA Sequencing analysis to exclude low quality cells as is considered best practice in such analyses. In this case, cells with less than 1251 genes and cells with mitochondrial proportions above $15 \%$ were discarded from further analysis. Further to this, genes expressed in less than 3 cells were removed. These criteria were established based upon previous preestablished standards combined with analysis from basic QC metrics.

Replication All replicates showed consistent results. For mouse studies reported here, each animal is an experimental unit. All studies were performed with at least 3 mice, with the exception of the TEADi mouse line due to mouse availability issues in the face of the pandemic.

Randomization Mice were randomly assigned to experimental groups

Blinding $\quad$ Blinding was not used in experiments in this manuscript, as differences in physical sample size made blinding impossible to experimenter with knowledge of the general project.

\section{Reporting for specific materials, systems and methods}


We require information from authors about some types of materials, experimental systems and methods used in many studies. Here, indicate whether each material, system or method listed is relevant to your study. If you are not sure if a list item applies to your research, read the appropriate section before selecting a response.

Materials \& experimental systems

\begin{tabular}{l|l}
\hline$n / a$ & Involved in the study \\
\hline & $\bigotimes$ Antibodies \\
$\square$ Eukaryotic cell lines \\
$\square$ & $\square$ Animals and other organisms \\
$\square$ & $\square$ Clinical data
\end{tabular}

\begin{tabular}{l|l}
\multicolumn{2}{l}{ Methods } \\
\hline n/a & Involved in the study \\
$\square$ & $\square$ ChIP-seq \\
$\square$ & $\bigotimes$ Flow cytometry \\
$\square$ & $\square$ MRI-based neuroimaging
\end{tabular}

\section{Antibodies}

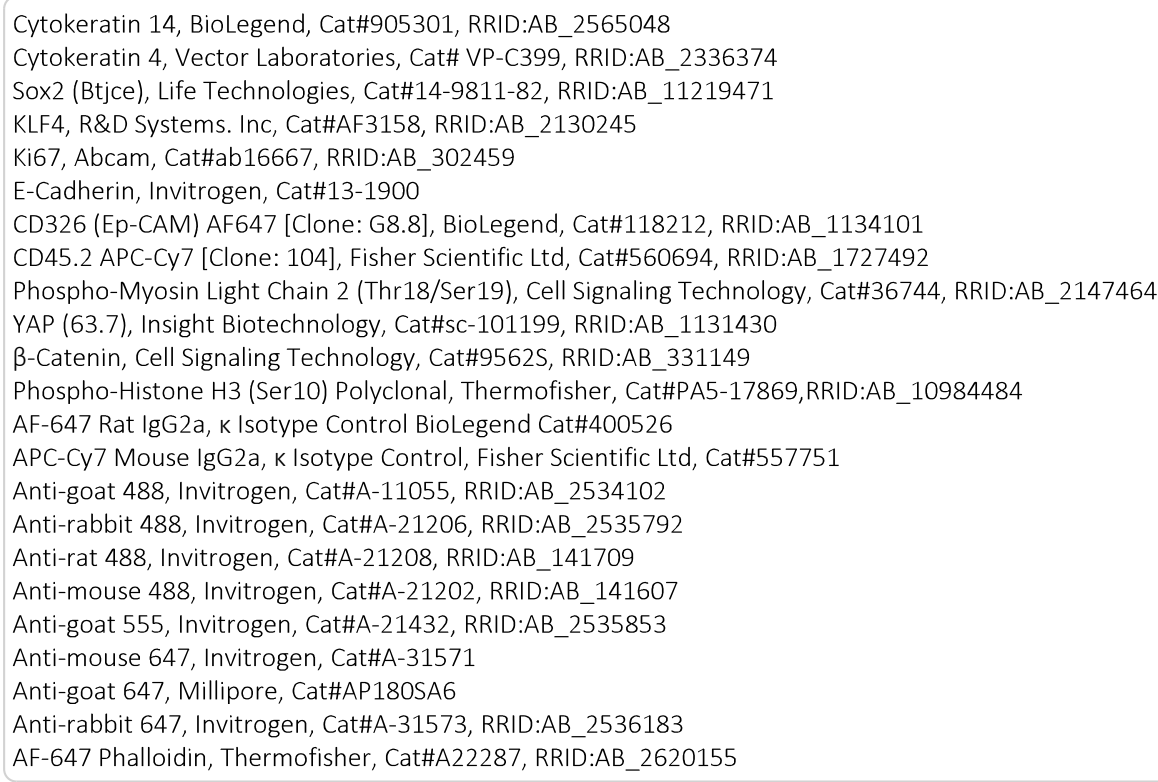

Validation

\section{Animals and other organisms}

Policy information about studies involving animals; ARRIVE guidelines recommended for reporting animal research 
Genetically engineered mouse lines used in the study include:

R26Fucci2aR, kindly provided by lan J. Jackson: Mort, R.L. et al. Fucci2a: a bicistronic cell cycle reporter that allows Cre mediated tissue specific expression in mice. Cell Cycle 13, 2681-2696 (2014).

tetO-YAP S127A, kindly provided by Jonas Larsson, stock \#006965, Jackson Laboratory, Jansson, L. \& Larsson, J. Normal hematopoietic stem cell function in mice with enforced expression of the Hippo signaling effector YAP1. PLoS One 7, e32013 (2012).

Krt5-rtTA;TEADi, kindly provided by Ramiro Iglesias-Bartolome: Yuan, Y. et al. YAP1/TAZ-TEAD transcriptional networks maintain skin homeostasis by regulating cell proliferation and limiting KLF4 activity. Nat Commun 11, 1472 (2020).

Rosa26-mT/mG, stock \# 007676, Jackson Laboratory: Muzumdar, M.D., Tasic, B., Miyamichi, K., Li, L. \& Luo, L. A global double-

fluorescent Cre reporter mouse. Genesis 45, 593-605 (2007).

Animals cohorts were used at exact post-natal days indicated, the adult cohort constitutes mice between 10-14 weeks of age, unless otherwise stated.

All strains were maintained in a C57BL/6 background.

All experiments comprised a mixture of male and female mice, with no gender specific differences observed. For single cell RNA sequencing (scRNAseq) experiments exclusively, only male mice were used in order to avoid confounding effects due to estrous cycle.

All animals were housed between 19-23C, 45-65\% humidity and a day/night light cycle of 12 hours on, 12 hours off.

Wild animals

Field-collected samples

Ethics oversight
This study did not involve wild animals.

This study did not involve samples collected from the field.

All experiments were approved by the local ethical review committees at the University of Cambridge unless stated below and conducted according to Home Office project license PPL70/8866 at the Wellcome Trust - Medical Research Council Cambridge Stem Cell Institute, Cambridge University.

Krt5-rtTA;TEADi studies were carried out according to approved protocols LCMB-034 and 19-329 from the NIH-Intramural Animal Care and Use Committee (ACUC) of the National Cancer Institute, NIH.

tetO-YAP S127A experiments were carried out under The National Animal Ethics Committee in Denmark reviewed and approved all animal procedures, permit number 2017-15-0201-01381.

Note that full information on the approval of the study protocol must also be provided in the manuscript

\section{Clinical data}

Policy information about clinical studies

All manuscripts should comply with the ICMJE guidelines for publication of clinical research and a completed CONSORT checklist must be included with all submissions.

Clinical trial registration

Provide the trial registration number from ClinicalTrials.gov or an equivalent agency.

Study protocol

Note where the full trial protocol can be accessed OR if not available, explain why.

Data collection

Describe the settings and locales of data collection, noting the time periods of recruitment and data collection.

Outcomes

Describe how you pre-defined primary and secondary outcome measures and how you assessed these measures.

\section{Flow Cytometry}

\section{Plots}

Confirm that:

\The axis labels state the marker and fluorochrome used (e.g. CD4-FITC).

Х The axis scales are clearly visible. Include numbers along axes only for bottom left plot of group (a 'group' is an analysis of identical markers).

$\bigotimes$ All plots are contour plots with outliers or pseudocolor plots.

$\bigotimes$ A numerical value for number of cells or percentage (with statistics) is provided.

\section{Methodology}

Sample preparation

Instrument

Software
All oesophageal samples were obtained as follows: All mice were culled and oesophagi were excised, taking as reference two anatomical landmarks (the cricoid cartilage and the opening of stomach fundus). Oesophageal tubes were used as such for stretching in vitro experiments, or were longitudinally opened and flattened under dissecting microscope for downstream processing/analysis.

BD FACSAria ${ }^{\mathrm{TM}}$ III cell sorter

FlowJo v10.6.2, BD, https://www.flowjo.com/solutions/flowjo/downloads

BD FACSDiva 8.0.1, https://www.bdbiosciences.com/us/instruments/clinical/software/flow-cytometry-acquisition/bd-facsdivasoftware/facsdiva-software-v-803-win-7-32-bit-os/p/659523 
EpCam+/CD45- viable cells represented on average $50.8 \%$ of viable cell fraction.

Gating strategy

Negative, positive and isotype controls were used to set up gates in this experiment.

$\bigotimes$ Tick this box to confirm that a figure exemplifying the gating strategy is provided in the Supplementary Information. 